\title{
On the $\mathcal{R}$-boundedness for the two phase problem: compressible-incompressible model problem
}

\author{
Takayuki Kubo $^{1 *}$, Yoshihiro Shibata ${ }^{2}$ and Kohei Soga ${ }^{3}$
}

\author{
"Correspondence: \\ tkubo@math.tsukuba.ac.jp \\ ${ }^{1}$ Division of Mathematics, Faculty of \\ Pure and Applied Sciences, \\ University of Tsukuba, 1-1-1 \\ Tennoudai, Tsukuba-shi, Ibaraki, \\ 305-8571, Japan \\ Full list of author information is \\ available at the end of the article
}

\begin{abstract}
The situation of this paper is that the Stokes equation for the compressible viscous fluid flow in the upper half-space is coupled via inhomogeneous interface conditions with the Stokes equations for the incompressible one in the lower half-space, which is the model problem for the evolution of compressible and incompressible viscous fluid flows with a sharp interface. We show the existence of $\mathcal{R}$-bounded solution operators to the corresponding generalized resolvent problem, which implies the generation of analytic semigroup and maximal $L_{p} L_{q}$ regularity for the corresponding time dependent problem with the help of the Weis' operator valued Fourier multiplier theorem. The problem was studied by Denisova (Interfaces Free Bound. 2(3):283-312, 2000) under some restriction on the viscosity coefficients and one of our purposes is to eliminate the assumption in (Denisova in Interfaces Free Bound. 2(3):283-312, 2000).

MSC: $35 \mathrm{Q} 35 ; 76 \mathrm{~T} 10$
\end{abstract}

Keywords: $\mathcal{R}$-boundedness; generalized resolvent problem; model problem; Stokes equations; compressible-incompressible two phase problem

\section{Introduction}

This paper is concerned with the evolution of compressible and incompressible viscous fluids separated by a sharp interface. Typical examples of the physical interpretation of our problem are the evolution of a bubble in an incompressible fluid flow, or a drop in a volume of gas. The problem is formulated as follows: Let $\Omega_{ \pm}$be two domains. The region $\Omega_{+}$is occupied by a compressible barotropic viscous fluid and the region $\Omega_{-}$by an incompressible viscous fluid. Let $\Gamma_{ \pm}$and $S_{ \pm}$be the boundaries of $\Omega_{ \pm}$such that $\Gamma_{ \pm} \cap S_{ \pm}=\emptyset$. We assume that $\Gamma_{+}=\Gamma_{-}$and $S_{+} \cap S_{-}=\emptyset$. We may assume that one of $S_{ \pm}$is an empty set, or that both of $S_{ \pm}$are empty sets. Let $\Gamma_{t}, S_{t-}$, and $\Omega_{t \pm}$ be the time evolutions of $\Gamma=\Gamma_{+}=\Gamma_{-}$, $S_{-}$, and $\Omega_{ \pm}$, respectively, where $t$ is the time variable. We assume that the two fluids are immiscible, so that $\Omega_{t+} \cap \Omega_{t-}=\emptyset$ for any $t \geq 0$. Moreover, we assume that no phase transitions occur and we do not consider the surface tension at the interface $\Gamma_{t}$ and the free boundary $S_{t+}$ for mathematical simplicity. Thus, the motion of the fluids is governed by

(c) 2014 Kubo et al: licensee Springer. This is an Open Access article distributed under the terms of the Creative Commons Attribution License (http://creativecommons.org/licenses/by/4.0), which permits unrestricted use, distribution, and reproduction in any medium, provided the original work is properly credited. 
the following system of equations:

$$
\left\{\begin{array}{lll}
\rho_{+}\left(\partial_{t} \mathbf{u}_{+}+\mathbf{u}_{+} \cdot \nabla \mathbf{u}_{+}\right)-\operatorname{Div} \mathbf{S}_{+}\left(\mathbf{u}_{+}, P\left(\rho_{+}\right)\right)=0, & \partial_{t} \rho_{+}+\operatorname{div}\left(\rho_{+} \mathbf{u}_{+}\right)=0 & \text { in } \Omega_{t+}, \\
\rho_{-}\left(\partial_{t} \mathbf{u}_{-}+\mathbf{u}_{-} \cdot \nabla \mathbf{u}_{-}\right)-\operatorname{Div} \mathbf{S}_{-}\left(\mathbf{u}_{-}, \pi_{-}\right)=0, & \operatorname{div} \mathbf{u}_{-}=0 & \text { in } \Omega_{t-}, \\
\left.\mathbf{S}_{+}\left(\mathbf{u}_{+}, P\left(\rho_{+}\right)\right) \mathbf{n}_{t}\right|_{\Gamma_{t+}}-\left.\mathbf{S}_{-}\left(\mathbf{u}_{-}, \pi_{-}\right) \mathbf{n}_{t}\right|_{\Gamma_{-}}=0, & \left.\mathbf{u}_{+}\right|_{\Gamma_{t+}}-\left.\mathbf{u}_{-}\right|_{\Gamma_{t-}}=0, & \\
\left.\mathbf{u}_{+}\right|_{S_{+}}=0,\left.\quad \mathbf{S}_{-}\left(\mathbf{u}_{-}, \pi_{-}\right) \mathbf{n}_{t-}\right|_{S_{-}}=0 &
\end{array}\right.
$$

for $t \in(0, T)$, subject to the initial conditions

$$
\left.\left(\mathbf{u}_{+}, \rho_{+}\right)\right|_{t=0}=\left(\mathbf{u}_{+0}, \rho_{+0}\right) \quad \text { in } \Omega_{+},\left.\quad \mathbf{u}_{-}\right|_{t=0}=\mathbf{u}_{-0} \quad \text { in } \Omega_{-} .
$$

Here, $\partial_{t}=\partial / \partial t, \rho_{-}$is a positive constant denoting the mass density of the reference domain $\Omega_{-}, P$ a pressure function, and $\mathbf{u}_{ \pm}=\left(u_{ \pm 1}, \ldots, u_{ \pm N}\right)(N \geq 2), \rho_{+}$and $\pi_{-}$are unknown velocities, scalar mass density and scalar pressure, respectively. Moreover, $\mathbf{S}_{ \pm}$are stress tensors defined by

$$
\begin{aligned}
& \mathbf{S}_{+}\left(\mathbf{u}_{+}, \pi_{+}\right)=\mu_{+} \mathbf{D}\left(\mathbf{u}_{+}\right)+\left(v_{+}-\mu_{+}\right) \operatorname{div} \mathbf{u}_{+} \mathbf{I}-\pi_{+} \mathbf{I}, \\
& \mathbf{S}_{-}\left(\mathbf{u}_{-}, p_{-}\right)=\mu_{-} \mathbf{D}\left(\mathbf{u}_{-}\right)-\pi_{-} \mathbf{I},
\end{aligned}
$$

where $\mathbf{D}(\mathbf{v})$ denotes the doubled strain tensor whose $(i, j)$ components are $D_{i j}(\mathbf{v})=\partial_{i} v_{j}+\partial_{j} v_{i}$ with $\partial_{i}=\partial / \partial x_{i}$ and we set $\operatorname{div} \mathbf{v}=\sum_{\ell=1}^{N} \partial_{\ell} v_{\ell}$ and $\mathbf{v} \cdot \nabla=\sum_{j=1}^{N} v_{j} \partial_{j}$ for any vector of functions $\mathbf{v}=\left(v_{1}, \ldots, v_{N}\right)$. And also, for any matrix field $K$ with $(i, j)$ components $K_{i j}$, the quantity $\operatorname{Div} K$ is an $N$-vector with components $\sum_{j=1}^{N} \partial_{j} K_{i j}$. Finally, $\mathbf{I}$ stands for the $N \times N$ identity matrix, $\mathbf{n}_{t}$ the unit normal to $\Gamma_{t}$ pointed from $\Omega_{t-}$ to $\Omega_{t+}, \mathbf{n}_{t-}$ the unit outward normal to $S_{t-}$, and $\mu_{ \pm}$and $v_{+}$are first and second viscosity coefficients, respectively, which are assumed to be constant and satisfy the condition

$$
\mu_{ \pm}>0, \quad v_{+}>0
$$

and $\left.f\right|_{\Gamma_{t \pm}}$ and $\left.f\right|_{S_{t-}}$ are defined by

$$
\left.f\right|_{\Gamma_{t \pm}}\left(x_{0}\right)=\lim _{\substack{x \in \Omega_{t \pm} \\ x \rightarrow x_{0}}} f(x) \quad \text { for } x_{0} \in \Gamma_{t},\left.\quad f\right|_{S_{t-}}=\lim _{\substack{x \in \Omega_{t-} \\ x \rightarrow x_{0}}} f(x) \quad \text { for } x_{0} \in S_{t} .
$$

Aside from the dynamical system (1.1), further kinematic conditions on $\Gamma_{t}$ and $S_{t}$ are satisfied, which give

$$
\Gamma_{t}=\left\{x \in \mathbb{R}^{N} \mid x=\mathbf{x}(\xi, t)\left(\xi \in \Gamma_{+}\right)\right\}, \quad S_{t}=\left\{x \in \mathbb{R}^{N} \mid x=\mathbf{x}(\xi, t)\left(\xi \in S_{-}\right)\right\} .
$$

Here, $\mathbf{x}=\mathbf{x}(\xi, t)$ is the solution to the Cauchy problem:

$$
\frac{d \mathbf{x}}{d t}=\mathbf{u}(\mathbf{x}, t) \quad(t>0),\left.\quad \mathbf{x}\right|_{t=0}=\xi \quad \text { in } \bar{\Omega}
$$

with $\mathbf{u}(x, t)=\mathbf{u}_{+}(x, t)$ for $x \in \Omega_{+}$and $\mathbf{u}(x, t)=\mathbf{u}_{-}(x, t)$ for $x \in \Omega_{-}$. This expresses the fact that the interface $\Gamma_{t}$ and the free boundary $S_{t}$ consist of the same particles for all $t>0$, 
which do not leave them and are not incident from $\Omega_{t \pm}$. In particular, we exclude the mass transportation through the interface $\Gamma_{t}$, because we assume that the two fluids are immiscible.

Denisova [1] studied a local in time unique existence theorem to problem (1.1) with surface tension on $\Gamma_{t}$ under the assumption that $\mu_{+}<\mu_{-}$and $\mu_{-} / \rho_{-}<\mu_{+} / R_{\infty}$ with some positive constant $R_{\infty}$ and that $\Omega_{+}$is bounded and $\Omega_{-}=\mathbb{R}^{N} \backslash \bar{\Omega}_{+}$. Here, $\rho_{-}$is a positive constant describing the mass density of the reference body $\Omega_{-}$. Thus, in [1], both of $S_{ \pm}$are empty sets. The purpose of our study is to prove local in time unique existence theorem in a general uniform domain under the assumption (1.4). Especially, the assumption on the viscosity coefficients is improved compared with Denisova [1] and widely accepted in the study of fluid dynamics.

As related topics about the two phase problem for the viscous fluid flows, the incompressible-incompressible case has been studied by [2-11] and the compressible-compressible case by $[12,13]$ as far as the authors know.

To prove a local in time existence theorem for (1.1), we transform (1.1) to the equations in fixed domains $\Omega_{ \pm}$by using the Lagrange transform ( $c$. Denisova [1]), so that the key step is to prove the maximal regularity for the linearized problem

$$
\begin{cases}\hat{\gamma}_{0+} \partial_{t} \mathbf{u}_{+}-\operatorname{Div} \mathbf{S}_{+}\left(\mathbf{u}_{+}, \hat{\gamma}_{2+} p_{+}\right)=\mathbf{g}_{+}, \quad \partial_{t} p_{+}+\hat{\gamma}_{1+} \operatorname{div} \mathbf{u}_{+}=f_{+} & \text {in } \Omega_{+}, \\ \gamma_{0-} \partial_{t} \mathbf{u}_{-}-\operatorname{Div} \mathbf{S}_{-}\left(\mathbf{u}_{-}, p_{-}\right)=\mathbf{g}_{-}, \quad \operatorname{div} \mathbf{u}_{-}=f_{-} & \text {in } \Omega_{-}, \\ \left.\mathbf{S}_{+}\left(\mathbf{u}_{+}, \hat{\gamma}_{2+} p_{+}\right) \mathbf{n}\right|_{\Gamma_{+}}-\left.\mathbf{S}_{-}\left(\mathbf{u}_{-}, p_{-}\right) \mathbf{n}\right|_{\Gamma_{-}}=\mathbf{h},\left.\quad \mathbf{u}_{+}\right|_{\Gamma}-\left.\mathbf{u}_{-}\right|_{\Gamma}=0, & \\ \left.\mathbf{u}_{+}\right|_{S_{+}}=0,\left.\quad \mathbf{S}_{-}\left(\mathbf{u}_{-}, p_{-}\right) \mathbf{n}_{-}\right|_{S_{-}}=\mathbf{h}_{-} & \end{cases}
$$

for any $t \in(0, T)$, subject to the initial conditions (1.2), where $\left.f\right|_{\Gamma_{ \pm}}\left(x_{0}\right)=\lim _{x \in \Omega_{ \pm}, x \rightarrow x_{0}} f(x)$ for $x_{0} \in \Gamma$. Here, $\gamma_{0-}$ is a positive constant and $\hat{\gamma}_{i_{+}}(i=0,1,2)$ are functions defined on $\bar{\Omega}_{+}$ such that

$$
\omega_{0} \leq \hat{\gamma}_{i^{+}}(x) \leq \omega_{1} \quad\left(x \in \bar{\Omega}_{+}\right), \quad \nabla \gamma_{i_{+}} \in L_{r}\left(\Omega_{+}\right)
$$

for $i=0,1,2$ with some positive constants $\omega_{0}$ and $\omega_{1}$ and with some exponent $r \in(N, \infty)$, and $\gamma_{0-}$ is a positive number describing the mass density of the flow occupied in $\Omega_{-}$. Our strategy of obtaining the maximal $L_{p}-L_{q}$ result for (1.6) is to show the existence of $\mathcal{R}$-bounded solution operator $R(\lambda)$ to the corresponding generalized resolvent problem:

$$
\begin{cases}\hat{\gamma}_{0+} \lambda \hat{\mathbf{u}}_{+}-\operatorname{Div} \mathbf{S}_{+}\left(\hat{\mathbf{u}}_{+}, \hat{\gamma}_{2+} \hat{p}_{+}\right)=\hat{\mathbf{g}}_{+}, \quad \lambda \hat{p}_{+}+\hat{\gamma}_{1+} \operatorname{div} \hat{\mathbf{u}}_{+}=\hat{f}_{+} & \text {in } \Omega_{+}, \\ \gamma_{0-} \lambda \hat{\mathbf{u}}_{-}-\operatorname{Div} \mathbf{S}_{-}\left(\hat{\mathbf{u}}_{-}, \hat{p}_{-}\right)=\hat{\mathbf{g}}_{-}, \quad \operatorname{div} \hat{\mathbf{u}}_{-}=\hat{f}_{-}, \quad \text { in } \Omega_{-}, \\ \left.\mathbf{S}_{+}\left(\mathbf{u}_{+}, \hat{\gamma}_{2+} \hat{p}_{+}\right) \mathbf{n}\right|_{\Gamma}-\left.\mathbf{S}_{-}\left(\hat{\mathbf{u}}_{-}, \hat{p}_{-}\right) \mathbf{n}\right|_{\Gamma_{t-}}=\left.\hat{\mathbf{h}}_{,} \quad \hat{\mathbf{u}}_{+}\right|_{\Gamma}-\left.\hat{\mathbf{u}}_{-}\right|_{\Gamma}=0, \\ \left.\hat{\mathbf{u}}_{+}\right|_{S_{+}}=0,\left.\quad \mathbf{S}_{-}\left(\hat{\mathbf{u}}_{-}, \hat{p}_{-}\right) \mathbf{n}_{-}\right|_{S_{-}}=\hat{\mathbf{h}}_{-} .\end{cases}
$$

Here, $\hat{f}$ denotes the Laplace transform of $f$ with respect to $t$. In fact, solutions $\hat{\mathbf{u}}_{ \pm}$and $\hat{p}_{ \pm}$ are represented by

$$
\left(\hat{\mathbf{u}}_{ \pm}, \hat{p}_{ \pm}\right)=R(\lambda)\left(\hat{f}_{ \pm}(\lambda), \hat{\mathbf{g}}_{ \pm}(\lambda), \hat{\mathbf{h}}(\lambda), \hat{\mathbf{h}}_{-}(\lambda)\right)
$$


so that roughly speaking, we can represent the solutions $\left(\mathbf{u}_{ \pm}(t), p_{ \pm}(t)\right)$ to the nonstationary problem (1.6) by

$$
\left(\mathbf{u}_{ \pm}(t), p_{ \pm}(t)\right)=\mathcal{L}^{-1}\left[R(\lambda)\left(\hat{f}_{ \pm}(\lambda), \hat{\mathbf{g}}_{ \pm}(\lambda), \hat{\mathbf{h}}(\lambda), \hat{\mathbf{h}}_{-}(\lambda)\right)\right](t)
$$

with Laplace inverse transform $\mathcal{L}^{-1}$. Thus, we get the maximal $L_{p}-L_{q}$ regularity result:

$$
\begin{aligned}
& \int_{0}^{\infty} e^{-p \gamma t}\left\{\left\|\left(p_{+}(\cdot, t), \partial_{t} p_{+}(\cdot, t)\right)\right\|_{W_{q}^{1}\left(\Omega_{+}\right)}+\sum_{\ell= \pm}\left(\left\|\partial_{t} \mathbf{u}_{\ell}(\cdot, t)\right\|_{L_{q}\left(\Omega_{\ell}\right)}+\left\|\mathbf{u}_{\ell}(\cdot, t)\right\|_{W_{q}^{2}\left(\Omega_{\ell}\right)}\right)\right\}^{p} d t \\
& \leq C\{\text { suitable norms of initial data and right members in }(1.6)\} \quad(1<p, q<\infty)
\end{aligned}
$$

for some positive constants $\gamma$ and $C$ with help of the Weis operator valued Fourier multiplier theorem [14]. To construct an $\mathcal{R}$-bounded solution operator to (1.7), problem (1.7) is reduced locally to the model problems in a neighborhood of an interface point as well as an interior point or a boundary point by using the localization technique and the partition of unity. The model problems for the interior point and boundary point have been studied, but the model problem for the interface point was studied only by Denisova [1] under some restriction on the viscosity coefficients. Moreover, she studied the problem in $L_{2}$ framework, so that the Plancherel formula is applicable. But our final goal is to treat the nonlinear problem (1.1) under (1.4) and (1.5) in the maximal $L_{p}-L_{q}$ regularity class, so that we need different ideas. Especially, the core of our approach is to construct an $\mathcal{R}$-bounded solution operator to (1.7). Thus, we construct the $\mathcal{R}$-bounded solution operator to (1.7) for the model problem in this paper, and in the forthcoming paper [15] we construct an $\mathcal{R}$-bounded solution operator to (1.7) in a domain. Moreover, in [15] the maximal $L_{p}-L_{q}$ regularity in a domain is derived automatically with the help of the Weis' operator valued Fourier multiplier theorem, so that a local in time unique existence theorem is proved by using the usual contraction mapping principle based on the maximal $L_{p}-L_{q}$ regularity.

Now we formulate our problem studied in this paper and state the main results. Let $\mathbb{R}_{+}^{N}$, $\mathbb{R}_{-}^{N}$, and $\mathbb{R}_{0}^{N}$ be the upper half-space, lower half-space and their boundary defined by

$$
\mathbb{R}_{ \pm}^{N}=\left\{x=\left(x_{1}, \ldots, x_{N}\right) \in \mathbb{R}^{N} \mid \pm x_{N}>0\right\}, \quad \mathbb{R}_{0}^{N}=\left\{x=\left(x_{1}, \ldots, x_{N}\right) \in \mathbb{R}^{N} \mid x_{N}=0\right\}
$$

In this paper, we consider the following model problem:

$$
\begin{cases}\lambda \mathbf{u}_{+}-\gamma_{0+}^{-1} \operatorname{Div} \mathbf{S}_{+}\left(\mathbf{u}_{+}, \gamma_{2+} p_{+}\right)=\mathbf{g}_{+}, \quad \lambda p_{+}+\gamma_{1_{+}} \operatorname{div} \mathbf{u}_{+}=f_{+} & \text {in } \mathbb{R}_{+}^{N}, \\ \lambda \mathbf{u}_{-}-\gamma_{0_{-}}^{-1} \operatorname{Div} \mathbf{S}_{-}\left(\mathbf{u}_{-}, p_{-}\right)=\mathbf{g}_{-}, \quad \operatorname{div} \mathbf{u}_{-}=0 & \text { in } \mathbb{R}_{-}^{N}, \\ \left.\mathbf{S}_{+}\left(\mathbf{u}_{+}, \gamma_{2+} p_{+}\right) \mathbf{n}\right|_{x_{N}=0+}-\left.\mathbf{S}_{-}\left(\mathbf{u}_{-}, p_{-}\right) \mathbf{n}\right|_{x_{N}=0-}=\mathbf{h}, & \\ \left.\mathbf{u}_{+}\right|_{x_{N}=0_{+}-\left.\mathbf{u}_{-}\right|_{x_{N}=0-}=\mathbf{k}} & \text { on } \mathbb{R}_{0}^{N} .\end{cases}
$$

Throughout the paper, $\mathbf{n}=(0, \ldots, 0,1), \gamma_{0 \pm}, \gamma_{1_{+}}$, and $\gamma_{2_{+}}$are fixed positive constants and the condition (1.4) holds. Substituting the relation $p_{+}=\left(f_{+}-\gamma_{1_{+}} \operatorname{div} \mathbf{u}_{+}\right) \lambda^{-1}$ into the equations in (1.8), we have

$$
\begin{aligned}
& \lambda \mathbf{u}_{+}-\gamma_{0_{+}}^{-1} \operatorname{Div}\left[\mu_{+} D\left(\mathbf{u}_{+}\right)+\left(v_{+}-\mu_{+}+\gamma_{1+} \gamma_{2+} \lambda^{-1}\right) \operatorname{div} \mathbf{u}_{+} \mathbf{I}\right]=\mathbf{g}_{+}-\gamma_{0_{+}}^{-1} \gamma_{2+} \lambda^{-1} \nabla f_{+}, \\
& \left.\left(\mu_{+} \mathbf{D}\left(\mathbf{u}_{+}\right)+\left(v_{+}-\mu_{+}+\gamma_{1_{+}} \gamma_{2_{+}} \lambda^{-1}\right) \operatorname{div} \mathbf{u}_{+} \mathbf{I}\right) \mathbf{n}\right|_{x_{N}=0+}-\left.\mathbf{S}_{-}\left(\mathbf{u}_{-}, p_{-}\right) \mathbf{n}\right|_{x_{N}=0-}=\mathbf{h}+\gamma_{2_{+}} \lambda^{-1} f_{+} \mathbf{n} .
\end{aligned}
$$


Thus, $\mathbf{g}_{+}-\gamma_{0_{+}}^{-1} \gamma_{2+} \lambda^{-1} \nabla f_{+}$and $\mathbf{h}+\gamma_{2+} \lambda^{-1} f_{+} \mathbf{n}$ being renamed $\mathbf{g}_{+}$and $\mathbf{h}$, respectively, and defining $\mathbf{S}_{\delta+}\left(\mathbf{u}_{+}\right)$by

$$
\mathbf{S}_{\delta+}\left(\mathbf{u}_{+}\right)=\mu_{+} \mathbf{D}\left(\mathbf{u}_{+}\right)+\left(v_{+}-\mu_{+}+\delta\right) \operatorname{div} \mathbf{u}_{+} \mathbf{I},
$$

mainly we consider the following problem:

$$
\begin{cases}\lambda \mathbf{u}_{+}-\gamma_{0+}^{-1} \operatorname{Div} \mathbf{S}_{\delta+}\left(\mathbf{u}_{+}\right)=\mathbf{g}_{+} & \text {in } \mathbb{R}_{+}^{N}, \\ \lambda \mathbf{u}_{-}-\gamma_{0-}^{-1} \operatorname{Div} \mathbf{S}_{-}\left(\mathbf{u}_{-}, p_{-}\right)=\mathbf{g}_{-}, \quad \operatorname{div} \mathbf{u}_{-}=0 & \text { in } \mathbb{R}_{-}^{N}, \\ \left.\mathbf{S}_{\delta+}\left(\mathbf{u}_{+}\right) \mathbf{n}\right|_{x_{N}=0+}-\left.\mathbf{S}_{-}\left(\mathbf{u}_{-}, p_{-}\right) \mathbf{n}\right|_{x_{N}=0-}=\mathbf{h}, & \\ \left.\mathbf{u}_{+}\right|_{x_{N}=0+}-\left.\mathbf{u}_{-}\right|_{x_{N}=0-}=\mathbf{k} & \text { on } \mathbb{R}_{0}^{N} .\end{cases}
$$

Here, $\delta$ is not only $\gamma_{1+} \gamma_{2+} \lambda^{-1}$ but also chosen as some complex number. More precisely, we consider the following three cases for $\delta$ and $\lambda$ :

(C1) $\delta=\gamma_{1+} \gamma_{2+} \lambda^{-1}, \lambda \in \Sigma_{\epsilon, \lambda_{0}} \cap K_{\epsilon}$.

(C2) $\delta \in \Sigma_{\epsilon}$ with $\operatorname{Re} \delta<0, \lambda \in \mathbb{C}$ with $|\lambda| \geq \lambda_{0}$ and $\operatorname{Re} \lambda \geq|\operatorname{Re} \delta / \operatorname{Im} \delta||\operatorname{Im} \lambda|$.

(C3) $\delta \in \Sigma_{\epsilon}$ with $\operatorname{Re} \delta \geq 0, \lambda \in \mathbb{C}$ with $|\lambda| \geq \lambda_{0}$ and $\operatorname{Re} \lambda \geq \lambda_{0}|\operatorname{Im} \lambda|$.

Here, $\Sigma_{\epsilon}=\{\lambda \in \mathbb{C} \backslash\{0\}|| \arg \lambda \mid \leq \pi-\epsilon\}$ with $0<\epsilon<\pi / 2, \Sigma_{\epsilon, \lambda_{0}}=\left\{\lambda \in \Sigma_{\epsilon}|| \lambda \mid \geq \lambda_{0}\right\}$ and

$$
K_{\epsilon}=\left\{\lambda \in \mathbb{C} \mid\left(\operatorname{Re} \lambda+\gamma_{1+} \gamma_{2+} \nu_{+}^{-1}+\epsilon\right)^{2}+(\operatorname{Im} \lambda)^{2} \geq\left(\gamma_{1+} \gamma_{2+} \nu_{+}^{-1}+\epsilon\right)^{2}\right\} .
$$

We define $\Gamma_{\epsilon, \lambda_{0}}$ by

$$
\Gamma_{\epsilon, \lambda_{0}}= \begin{cases}\Sigma_{\epsilon, \lambda_{0}} \cap K_{\epsilon} & \text { in case of (C1), } \\ \left\{\lambda \in \mathbb{C}|| \lambda\left|\geq \lambda_{0}, \operatorname{Re} \lambda \geq\right| \operatorname{Re} \delta / \operatorname{Im} \delta|| \operatorname{Im} \lambda \mid\right\} & \text { in case of (C2), } \\ \left\{\lambda \in \mathbb{C}|| \lambda\left|\geq \lambda_{0}, \operatorname{Re} \lambda \geq \lambda_{0}\right| \operatorname{Im} \lambda \mid\right\} & \text { in case of (C3). }\end{cases}
$$

The case (C1) is used to prove the existence of $\mathcal{R}$-bounded solution operator to (1.8) and the cases (C2) and (C3) are used for some homotopic argument in proving the exponential stability of analytic semigroup in a bounded domain. Such homotopic argument already appeared in [16] and [17] in the non-slip condition case. In (C2), we note that $\operatorname{Im} \delta \neq 0$ when $\delta \in \Sigma_{\epsilon}$ with $\operatorname{Re} \delta<0$.

In case (C1), $|\delta|=\left|\gamma_{1+} \gamma_{2+} \lambda^{-1}\right| \leq \gamma_{1+} \gamma_{2+} \lambda_{0}^{-1}$. On the other hand, in cases of (C2) and (C3), we assume that $|\delta| \leq \delta_{0}$ for some $\delta_{0}>0$. Thus, we assume that

$$
|\delta| \leq \max \left(\gamma_{1+} \gamma_{2+} \lambda_{0}^{-1}, \delta_{0}\right) .
$$

We may include the case where $\gamma_{1+} \gamma_{2+}=0$ in (1.9), which is corresponding to the Lamé system. We may also consider the case where $\operatorname{div} \mathbf{u}_{-}=f_{-}$in (1.8) under the condition that $f_{-} \in W_{q}^{1}\left(\mathbb{R}_{-}^{N}\right)$ and $f_{-}=\operatorname{div} F_{-}$with some $F_{-} \in L_{q}\left(\mathbb{R}_{-}^{N}\right)^{N}$. In fact, first we solve the equation $\operatorname{div} \mathbf{u}_{-}=f_{-}$in $\mathbb{R}_{-}^{N}$, which transfers the problem to the case where $f_{-}=0$ (cf. Shibata [18, Section 3]). Thus, we only consider the case where $f_{-}=0$ in this paper for the sake of simplicity.

Before stating our main results, we introduce several symbols and functional spaces used throughout the paper. For the differentiations of scalar $f$ and $N$-vector $\mathbf{g}=\left(g_{1}, \ldots, g_{N}\right)$, we 
use the following symbols:

$$
\begin{aligned}
& \nabla f=\left(\partial_{1} f, \ldots, \partial_{N} f\right), \quad \nabla^{2} f=\left(\partial_{i} \partial_{j} f \mid i, j=1, \ldots, N\right), \\
& \nabla \mathbf{g}=\left(\partial_{i} g_{j} \mid i, j=1, \ldots, N\right), \quad \nabla^{2} \mathbf{g}=\left(\partial_{i} \partial_{j} g_{k} \mid i, j, k=1, \ldots, N\right) .
\end{aligned}
$$

For any Banach space $X$ with norm $\|\cdot\|_{X}, X^{d}$ denotes the $d$-product space of $X$, while its norm is denoted by $\|\cdot\|_{X}$ instead of $\|\cdot\|_{X^{d}}$ for the sake of simplicity. For any domain $D, L_{q}(D)$, and $W_{q}^{m}(D)$ denote the usual Lebesgue space and Sobolev space, while $\|\cdot\|_{L_{q}(D)}$ and $\|\cdot\|_{W_{q}^{m}(D)}$ denote their norms, respectively. We set $\hat{W}_{q}^{1}\left(\mathbb{R}_{-}^{N}\right)=\left\{\theta \in L_{q, \text { loc }}\left(\mathbb{R}_{-}^{N}\right) \mid\right.$ $\left.\nabla \theta \in L_{q}\left(\mathbb{R}_{-}^{N}\right)^{N}\right\}$. For any two Banach spaces $X$ and $Y, \mathcal{L}(X, Y)$ denotes the set of all bounded linear operators from $X$ into $Y$. $\operatorname{Hol}(U, X)$ denotes the set of all $X$-valued holomorphic functions defined on $U$. The letter $C$ denotes generic constants and the constant $C_{a, b, \ldots}$ depends on $a, b, \ldots$. The values of constants $C$ and $C_{a, b, \ldots}$ may change from line to line. $\mathbb{N}$ and $\mathbb{C}$ denote the set of all natural numbers and complex numbers, respectively, and we set $\mathbb{N}_{0}=\mathbb{N} \cup\{0\}$. For any multi-index $\alpha=\left(\alpha_{1}, \ldots, \alpha_{N}\right) \in \mathbb{N}_{0}^{N}$, we set $\partial_{x}^{\alpha}=\left(\partial / \partial x_{1}\right)^{\alpha_{1}} \ldots\left(\partial / \partial x_{N}\right)_{N}^{\alpha_{N}}$.

We introduce the definition of $\mathcal{R}$-boundedness.

Definition 1.1 A family of operators $\mathcal{T} \subset \mathcal{L}(X, Y)$ is called $\mathcal{R}$-bounded on $\mathcal{L}(X, Y)$, if there exist constants $C>0$ and $q \in[1, \infty)$ such that for any $n \in \mathbb{N},\left\{T_{j}\right\}_{j=1}^{n} \subset \mathcal{T},\left\{x_{j}\right\}_{j=1}^{n} \subset X$ and sequences $\left\{r_{j}(u)\right\}_{j=1}^{n}$ of independent, symmetric, $\{-1,1\}$-valued random variables on $[0,1]$ we have the inequality

$$
\left\{\int_{0}^{1}\left\|\sum_{j=1}^{n} r_{j}(u) T_{j} x_{j}\right\|_{Y}^{q} d u\right\}^{\frac{1}{q}} \leq C\left\{\int_{0}^{1}\left\|\sum_{j=1}^{n} r_{j}(u) x_{j}\right\|_{X}^{q} d u\right\}^{\frac{1}{q}} .
$$

The smallest such $C$ is called $\mathcal{R}$-bound of $\mathcal{T}$, which is denoted by $\mathcal{R}_{\mathcal{L}(X, Y)}(\mathcal{T})$.

The following theorem is our main result in this paper.

Theorem 1.2 Let $1<q<\infty, 0<\epsilon<\pi / 2$ and $\lambda_{0}>0$. Let $\Gamma_{\epsilon, \lambda_{0}}$ be the sets defined in (1.12). Let $X_{q}$ and $\mathcal{X}_{q}$ be the sets defined by

$$
\begin{aligned}
X_{q}= & \left\{\left(\mathbf{g}_{+}, \mathbf{g}_{-}, \mathbf{h}, \mathbf{k}\right) \mid \mathbf{g}_{ \pm} \in L_{q}\left(\mathbb{R}_{ \pm}^{N}\right)^{N}, \mathbf{h} \in W_{q}^{1}\left(\mathbb{R}^{N}\right)^{N}, \mathbf{k} \in W_{q}^{2}\left(\mathbb{R}^{N}\right)^{N}\right\}, \\
\mathcal{X}_{q}= & \left\{F=\left(F_{1+}, F_{1-}, F_{2}, F_{3}, F_{4}, F_{5}, F_{6}\right) \mid\right. \\
& \left.F_{1 \pm} \in L_{q}\left(\mathbb{R}_{ \pm}^{N}\right), F_{2}, F_{5} \in L_{q}\left(\mathbb{R}^{N}\right)^{N^{2}}, F_{3}, F_{6} \in L_{q}\left(\mathbb{R}^{N}\right)^{N}, F_{4} \in L_{q}\left(\mathbb{R}^{N}\right)^{N^{3}}\right\} .
\end{aligned}
$$

Then there exist operator families

$$
\mathcal{A}_{ \pm}(\lambda) \in \operatorname{Hol}\left(\Gamma_{\epsilon, \lambda_{0}}, \mathcal{L}\left(\mathcal{X}_{q}, W_{q}^{2}\left(\mathbb{R}_{ \pm}^{N}\right)^{N}\right)\right), \quad \mathcal{P}_{-}(\lambda) \in \operatorname{Hol}\left(\Gamma_{\epsilon, \lambda_{0}}, \mathcal{L}\left(\mathcal{X}_{q}, \hat{W}_{q}^{1}\left(\mathbb{R}_{-}^{N}\right)\right)\right)
$$

such that $\mathbf{u}_{ \pm}=\mathcal{A}_{ \pm}(\lambda) F_{\lambda}\left(\mathbf{g}_{+}, \mathbf{g}_{-}, \mathbf{h}, \mathbf{k}\right)$ and $p_{-}=\mathcal{P}_{-}(\lambda) F_{\lambda}\left(\mathbf{g}_{+}, \mathbf{g}_{-}, \mathbf{h}, \mathbf{k}\right)$ solve problem $(1.10)$ uniquely for any $\left(\mathbf{g}_{+}, \mathbf{g}_{-}, \mathbf{h}, \mathbf{k}\right) \in X_{q}$ and $\lambda \in \Gamma_{\epsilon, \lambda_{0}}$, where $F_{\lambda}\left(\mathbf{g}_{+}, \mathbf{g}_{-}, \mathbf{h}, \mathbf{k}\right)=\left(\mathbf{g}_{+}, \mathbf{g}_{-}, \nabla \mathbf{h}, \lambda^{1 / 2} \mathbf{h}_{ \pm}\right.$, $\left.\nabla^{2} \mathbf{k}, \lambda^{1 / 2} \nabla \mathbf{k}, \lambda \mathbf{k}\right)$. 
Moreover, there exists a constant $C$ depending on $\epsilon, q$, and $N$ such that

$$
\begin{aligned}
& \mathcal{R}_{\mathcal{L}\left(\mathcal{X}_{q}, L_{q}\left(\mathbb{R}_{ \pm}^{N}\right)^{\tilde{N}}\right)}\left(\left\{\left(\tau \partial_{\tau}\right)^{\ell}\left(G_{\lambda} \mathcal{A}_{ \pm}(\lambda)\right) \mid \lambda \in \Gamma_{\epsilon, \lambda_{0}}\right\}\right) \leq C \quad(\ell=0,1) \\
& \mathcal{R}_{\mathcal{L}\left(\mathcal{X}_{q}, L_{q}\left(\mathbb{R}_{-}^{N}\right)^{N}\right)}\left(\left\{\left(\tau \partial_{\tau}\right)^{\ell}\left(\nabla \mathcal{P}_{-}(\lambda)\right) \mid \lambda \in \Lambda_{\epsilon, \lambda_{0}}\right\}\right) \leq C \quad(\ell=0,1)
\end{aligned}
$$

with $\tilde{N}=N^{3}+N^{2}+2 N$ and $\lambda=\gamma+i \tau$, where $G_{\lambda}$ is an operator defined by $G_{\lambda} \mathbf{u}=$ $\left(\lambda \mathbf{u}, \gamma \mathbf{u}, \lambda^{1 / 2} \nabla \mathbf{u}, \nabla^{2} \mathbf{u}\right)$.

Setting $p_{+}=\lambda^{-1}\left(f_{+}-\gamma_{1+} \operatorname{div} \mathbf{u}_{+}\right)$in (1.8), we have the following theorem concerning problem (1.8) immediately with the help of Theorem 1.2.

Theorem 1.3 Let $1<q<\infty, 0<\epsilon<\pi / 2$ and $\lambda_{0}>0$. Let $\Gamma_{\epsilon, \lambda_{0}}$ be the sets defined in (1.12). Set

$$
\begin{aligned}
& Y_{q}=\left\{\left(f_{+}, \mathbf{g}_{+}, \mathbf{g}_{-}, \mathbf{h}, \mathbf{k}\right) \mid f_{+} \in W_{q}^{1}\left(\mathbb{R}_{+}^{N}\right),\left(\mathbf{g}_{+}, \mathbf{g}_{-}, \mathbf{h}, \mathbf{k}\right) \in X_{q}\right\}, \\
& \mathcal{Y}_{q}=\left\{\left(F_{0}, F_{1^{+}}, F_{1-}, F_{2}, F_{3}, F_{4}, F_{5}, F_{6}\right) \mid F_{0} \in W_{q}^{1}\left(\mathbb{R}_{+}^{N}\right),\left(F_{1_{+}}, F_{1-}, F_{2}, F_{3}, F_{4}, F_{5}, F_{6}\right) \in \mathcal{X}_{q}\right\} .
\end{aligned}
$$

Then there exist operator families

$$
\begin{aligned}
& \mathcal{P}_{+}(\lambda) \in \operatorname{Hol}\left(\Lambda_{\epsilon, \lambda_{0}}, \mathcal{L}\left(\mathcal{Y}_{q}, W_{q}^{1}\left(\mathbb{R}_{+}^{N}\right)\right)\right), \quad \mathcal{U}_{ \pm}(\lambda) \in \operatorname{Hol}\left(\Lambda_{\epsilon, \lambda_{0}}, \mathcal{L}\left(\mathcal{Y}_{q}, W_{q}^{2}\left(\mathbb{R}_{ \pm}^{N}\right)^{N}\right)\right), \\
& \mathcal{P}_{-}(\lambda) \in \operatorname{Hol}\left(\Lambda_{\epsilon, \lambda_{0}}, \mathcal{L}\left(\mathcal{Y}_{q}, \hat{W}_{q}^{1}\left(\mathbb{R}_{-}^{N}\right)\right)\right)
\end{aligned}
$$

such that for any $\left(f_{+}, \mathbf{g}_{+}, \mathbf{g}_{-}, \mathbf{h}, \mathbf{k}\right) \in Y_{q}$ and $\lambda \in \Lambda_{\epsilon, \lambda_{0}}$,

$$
\begin{aligned}
& p_{+}=\mathcal{P}_{+}(\lambda) F_{\lambda}^{\prime}\left(f_{+}, \mathbf{g}_{+}, \mathbf{g}_{-}, \mathbf{h}, \mathbf{k}\right), \quad \mathbf{u}_{ \pm}=\mathcal{U}_{ \pm}(\lambda) F_{\lambda}^{\prime}\left(f_{+}, \mathbf{g}_{+}, \mathbf{g}_{-}, \mathbf{h}, \mathbf{k}\right), \\
& p_{-}=\mathcal{P}_{-}(\lambda) F_{\lambda}^{\prime}\left(f_{+}, \mathbf{g}_{+}, \mathbf{g}_{-}, \mathbf{h}, \mathbf{k}\right)
\end{aligned}
$$

solve problem (1.8) uniquely, where $F_{\lambda}^{\prime}\left(f_{+}, \mathbf{g}_{+}, \mathbf{g}_{-}, \mathbf{h}, \mathbf{k}\right)=\left(f_{+}, \mathbf{g}_{+}, \mathbf{g}_{-}, \nabla \mathbf{h}, \lambda^{1 / 2} \mathbf{h}, \nabla^{2} \mathbf{k}, \lambda^{1 / 2} \nabla \mathbf{k}\right.$, $\lambda \mathbf{k})$.

Moreover, there exists a constant $C$ depending on $\epsilon, \lambda_{0}, q$, and $N$ such that

$$
\begin{aligned}
& \mathcal{R}_{\mathcal{L}\left(\mathcal{Y}_{q}, W_{q}^{1}\left(\mathbb{R}_{+}^{N}\right)^{2}\right)}\left(\left\{\left(\tau \partial_{\tau}\right)^{\ell}\left\{(\lambda, \gamma) \mathcal{P}_{+}(\lambda)\right\} \mid \lambda \in \Gamma_{\epsilon, \lambda_{0}}\right\}\right) \leq C \quad(\ell=0,1), \\
& \mathcal{R}_{\mathcal{L}\left(\mathcal{Y}_{q}, L_{q}\left(\mathbb{R}_{ \pm}^{N}\right)^{\tilde{N}}\right)}\left(\left\{\left(\tau \partial_{\tau}\right)^{\ell}\left(G_{\lambda} \mathcal{U}_{ \pm}(\lambda)\right) \mid \lambda \in \Gamma_{\epsilon, \lambda_{0}}\right\}\right) \leq C \quad(\ell=0,1), \\
& \mathcal{R}_{\mathcal{L}\left(\mathcal{Y}_{q}, L_{q}\left(\mathbb{R}_{-}^{N}\right)^{N}\right)}\left(\left\{\left(\tau \partial_{\tau}\right)^{\ell}\left(\nabla \mathcal{P}_{-}(\lambda)\right) \mid \lambda \in \Gamma_{\epsilon, \lambda_{0}}\right\}\right) \leq C \quad(\ell=0,1) .
\end{aligned}
$$

\section{Solution formulas for the model problem}

To prove Theorem 1.2, first we consider problem (1.10) with $\mathbf{g}_{ \pm}=0$ in this section as a model problem, that is, we consider the following equations:

$$
\begin{cases}\lambda \mathbf{u}_{+}-\gamma_{0+}^{-1} \operatorname{Div} \mathbf{S}_{\delta+}\left(\mathbf{u}_{+}\right)=0 & \text { in } \mathbb{R}_{+}^{N}, \\ \lambda \mathbf{u}_{-}-\gamma_{0-}^{-1} \operatorname{Div} \mathbf{S}_{-}\left(\mathbf{u}_{-}, p_{-}\right)=0, \quad \operatorname{div} \mathbf{u}_{-}=0 & \text { in } \mathbb{R}_{-}^{N}, \\ \left.\mathbf{S}_{\delta+}\left(\mathbf{u}_{+}\right) \mathbf{n}\right|_{x_{N}=0+}-\left.\mathbf{S}_{-}\left(\mathbf{u}_{-}, p_{-}\right) \mathbf{n}\right|_{x_{N}=0-}=\mathbf{h},\left.\quad \mathbf{u}_{+}\right|_{x_{N}=0+}-\left.\mathbf{u}_{-}\right|_{x_{N}=0-}=\mathbf{k} & \text { on } \mathbb{R}_{0}^{N} .\end{cases}
$$


Let $\hat{v}=\mathcal{F}_{x^{\prime}}[v]\left(\xi^{\prime}, x_{N}\right)$ denote the partial Fourier transform with respect to the tangential variable $x^{\prime}=\left(x_{1}, \ldots, x_{N-1}\right)$ with $\xi^{\prime}=\left(\xi_{1}, \ldots, \xi_{N-1}\right)$ defined by $\mathcal{F}_{x^{\prime}}[v]\left(\xi^{\prime}, x_{N}\right)=\int_{\mathbb{R}^{N-1}} e^{-i x^{\prime} \cdot \xi^{\prime}} v\left(x^{\prime}\right.$, $\left.x_{N}\right) d x^{\prime}$. Using the formulas

$\operatorname{Div} \mathbf{S}_{\delta+}\left(\mathbf{u}_{+}\right)=\mu_{+} \Delta \mathbf{u}_{+}+\left(v_{+}+\delta\right) \nabla \operatorname{div} \mathbf{u}_{+}, \quad \operatorname{Div} \mathbf{S}_{-}\left(\mathbf{u}_{-}, p_{-}\right)=\mu_{-} \Delta \mathbf{u}_{-}-\nabla p_{-}$

and applying the partial Fourier transform to (2.1), we transfer problem (2.1) to the ordinary differential equations

$$
\begin{cases}\lambda \hat{u}_{+j}+\gamma_{0+}^{-1} \mu_{+}\left|\xi^{\prime}\right|^{2} \hat{u}_{+j}-\gamma_{0+}^{-1} \mu_{+} D_{N}^{2} \hat{u}_{+j} & \\ \quad-\gamma_{0+}^{-1}\left(v_{+}+\delta\right) i \xi_{j}\left(i \xi^{\prime} \cdot \hat{u}_{+}^{\prime}+D_{N} \hat{u}_{+N}\right)=0 & \text { for } x_{N}>0 \\ \lambda \hat{u}_{+N}+\gamma_{0+}^{-1} \mu_{+}\left|\xi^{\prime}\right|^{2} \hat{u}_{+N}-\gamma_{0+}^{-1} \mu_{+} D_{N}^{2} \hat{u}_{+N} & \\ \quad-\gamma_{0+}^{-1}\left(v_{+}+\delta\right) D_{N}\left(i \xi^{\prime} \cdot \hat{u}_{+}^{\prime}+D_{N} \hat{u}_{+N}\right)=0 & \text { for } x_{N}>0, \\ \lambda \hat{u}_{-j}+\gamma_{0-}^{-1} \mu_{-}\left|\xi^{\prime}\right|^{2} \hat{u}_{-j}-\gamma_{0-}^{-1} \mu_{-} D_{N}^{2} \hat{u}_{-j}+\gamma_{0-}^{-1} i \xi_{j} \hat{p}_{-}=0 & \text { for } x_{N}<0 \\ \lambda \hat{u}_{-N}+\gamma_{0-}^{-1} \mu_{-}\left|\xi^{\prime}\right|^{2} \hat{u}_{-N}-\gamma_{0-}^{-1} \mu_{-} D_{N}^{2} \hat{u}_{-N}+\gamma_{0-}^{-1} D_{N} \hat{p}_{-}=0 & \text { for } x_{N}<0 \\ i \xi^{\prime} \cdot \hat{u}_{-}^{\prime}+D_{N} \hat{u}_{-N}=0 & \text { for } x_{N}<0\end{cases}
$$

subject to the boundary conditions

$$
\left\{\begin{array}{l}
\left.\mu_{+}\left(D_{N} \hat{u}_{+j}+i \xi_{j} \hat{u}_{+N}\right)\right|_{x_{N}=0+}-\left.\mu_{-}\left(D_{N} \hat{u}_{-j}+i \xi_{j} \hat{u}_{-N}\right)\right|_{x_{N}=0-}=\hat{h}_{j}(0), \\
2 \mu_{+} D_{N} \hat{u}_{+N}+\left.\left(v_{+}-\mu_{+}+\delta\right)\left(i \xi^{\prime} \cdot \hat{u}_{+}^{\prime}+D_{N} \hat{u}_{+N}\right)\right|_{x_{N}=0+} \\
\quad-\left.\left(2 \mu_{-} D_{N} \hat{u}_{-N}-\hat{p}_{-}\right)\right|_{x_{N}=0-}=\hat{h}_{N}(0), \\
\hat{u}_{+J}(0+)-\hat{u}_{-J}(0-)=\hat{k}_{J}(0),
\end{array}\right.
$$

where $D_{N}=d / d x_{N}$ and $i \xi^{\prime} \cdot \hat{v}^{\prime}=\sum_{\ell=1}^{N-1} i \xi_{\ell} \hat{v}_{j}$ for $\mathbf{v}=\left(v_{1}, \ldots, v_{N-1}, v_{N}\right)$. Here and in the following, $j$ and $J$ run from 1 through $N-1$ and $N$, respectively. Applying the divergence to the first and second equations in (2.1), we have $\lambda \operatorname{div} \mathbf{u}_{+}-\gamma_{0_{+}}^{-1}\left(\mu_{+}+v_{+}+\delta\right) \Delta \operatorname{div} \mathbf{u}_{+}=0$ in $\mathbb{R}_{+}^{N}$ and $\Delta p_{-}=0$ in $\mathbb{R}_{-}^{N}$, so that

$$
\begin{aligned}
& \left(\lambda-\gamma_{0+}^{-1}\left(\mu_{+}+v_{+}+\delta\right) \Delta\right)\left(\lambda-\gamma_{0_{+}}^{-1} \mu_{+} \Delta\right) \mathbf{u}_{+}=0 \quad \text { in } \mathbb{R}_{+}^{N}, \\
& \left(\lambda-\gamma_{0-}^{-1} \Delta\right) \Delta \mathbf{u}_{-}=0 \quad \text { in } \mathbb{R}_{-}^{N} .
\end{aligned}
$$

Thus, the characteristic roots of (2.2) are

$$
A_{+}=\sqrt{\gamma_{0+}\left(\mu_{+}+v_{+}+\delta\right)^{-1} \lambda+A^{2}}, \quad B_{ \pm}=\sqrt{\gamma_{0 \pm}\left(\mu_{ \pm}\right)^{-1} \lambda+A^{2}}, \quad A=\left|\xi^{\prime}\right| .
$$

To state our solution formulas of problem: (2.2)-(2.3), we introduce some classes of multipliers.

Definition 2.1 Let $s$ be a real number and let $\Gamma_{\epsilon, \lambda_{0}}$ be the set defined in (1.12). Set

$$
\tilde{\Gamma}_{\epsilon, \lambda_{0}}=\left\{\left(\lambda, \xi^{\prime}\right) \mid \lambda=\gamma+i \tau \in \Gamma_{\epsilon, \lambda_{0}}, \xi^{\prime}=\left(\xi_{1}, \ldots, \xi_{N-1}\right) \in \mathbb{R}^{N-1} \backslash\{0\}\right\} .
$$

Let $m\left(\lambda, \xi^{\prime}\right)$ be a function defined on $\tilde{\Gamma}_{\epsilon, \lambda_{0}}$. 
(1) $m\left(\lambda, \xi^{\prime}\right)$ is called a multiplier of order $s$ with type 1 if for any multi-index $\kappa^{\prime}=\left(\kappa_{1}, \ldots, \kappa_{N-1}\right) \in \mathbb{N}_{0}^{N-1}$ and $\left(\lambda, \xi^{\prime}\right) \in \tilde{\Gamma}_{\epsilon, \lambda_{0}}$ there exists a constant $C_{\kappa^{\prime}}$ depending on $\kappa^{\prime}, \lambda_{0}, \epsilon, \mu_{ \pm}, \nu_{+}, \gamma_{0_{-}}$, and $\gamma_{i+}(i=0,1,2)$ such that we have the estimates

$$
\begin{aligned}
& \left|\partial_{\xi^{\prime}}^{\kappa^{\prime}} m\left(\lambda, \xi^{\prime}\right)\right| \leq C_{\alpha^{\prime}}\left(|\lambda|^{1 / 2}+A\right)^{s-\left|\kappa^{\prime}\right|}, \\
& \left|\partial_{\xi^{\prime}}^{\kappa^{\prime}}\left(\tau \frac{\partial m}{\partial \tau}\left(\lambda, \xi^{\prime}\right)\right)\right| \leq C_{\kappa^{\prime}}\left(|\lambda|^{1 / 2}+A\right)^{s-\left|\kappa^{\prime}\right|} .
\end{aligned}
$$

(2) $m\left(\lambda, \xi^{\prime}\right)$ is called a multiplier of order $s$ with type 2 if for any multi-index $\kappa^{\prime}=\left(\kappa_{1}, \ldots, \kappa_{N-1}\right) \in \mathbb{N}_{0}^{N-1}$ and $\left(\lambda, \xi^{\prime}\right) \in \tilde{\Gamma}_{\epsilon, \lambda_{0}}$ there exists a constant $C_{\kappa^{\prime}}$ depending on $\kappa^{\prime}, \lambda_{0}, \epsilon, \mu_{ \pm}, \nu_{+}, \gamma_{0-}$, and $\gamma_{i+}(i=0,1,2)$ such that we have the estimates

$$
\begin{aligned}
& \left|\partial_{\xi^{\prime}}^{\kappa^{\prime}} m\left(\lambda, \xi^{\prime}\right)\right| \leq C_{\kappa^{\prime}}\left(|\lambda|^{1 / 2}+A\right)^{s} A^{-\left|\kappa^{\prime}\right|}, \\
& \left|\partial_{\xi^{\prime}}^{\kappa^{\prime}}\left(\tau \frac{\partial m}{\partial \tau}\left(\lambda, \xi^{\prime}\right)\right)\right| \leq C_{\kappa^{\prime}}\left(|\lambda|^{1 / 2}+A\right)^{s} A^{-\left|\kappa^{\prime}\right|} .
\end{aligned}
$$

Let $\mathbf{M}_{s, i}$ be the set of all multipliers of order $s$ with type $i(i=1,2)$.

Obviously, $\mathbf{M}_{s, i}$ are vector spaces on $\mathbb{C}$. Moreover, by the fact $\left|\lambda^{1 / 2}+A\right|^{-\left|\alpha^{\prime}\right|} \leq A^{-\left|\alpha^{\prime}\right|}$ and the Leibniz rule, we have the following lemma immediately.

Lemma 2.2 Let $s_{1}, s_{2}$ be two real numbers. Then the following three assertions hold.

(1) Given $m_{i} \in \mathbf{M}_{s_{i}, 1}(i=1,2)$, we have $m_{1} m_{2} \in \mathbf{M}_{s_{1}+s_{2}, 1}$.

(2) Given $\ell_{i} \in \mathbf{M}_{s_{i}, i}(i=1,2)$, we have $\ell_{1} \ell_{2} \in \mathbf{M}_{s_{1}+s_{2}, 2}$.

(3) Given $n_{i} \in \mathbf{M}_{s_{i}, 2}(i=1,2)$, we have $m_{1} m_{2} \in \mathbf{M}_{s_{1}+s_{2}, 2}$.

Remark 2.3 We see easily that $i \xi_{j} \in \mathbf{M}_{1,2}(j=1, \ldots, N-1), A \in \mathbf{M}_{1,2}$, and $A^{-1} \in \mathbf{M}_{-1,2}$. Especially, $i \xi_{j} / A \in \mathbf{M}_{0,2}$. Moreover, $\mathbf{M}_{s, 1} \subset \mathbf{M}_{s, 2}$ for any $s \in \mathbb{R}$.

In this section we show the following solution formulas for problem (2.2)-(2.3):

$$
\begin{aligned}
& \hat{u}_{+J}=\sum_{k=1}^{4} \hat{u}_{J k}^{+}, \quad \hat{u}_{-J}=\sum_{k=1}^{3} \hat{u}_{J k}^{-}, \quad \hat{p}=e^{A x_{N}} \sum_{\ell=1}^{N}\left[p_{\ell, 0}^{-} \hat{h}_{\ell}(0)+p_{\ell, 1}^{-} \hat{k}_{\ell}(0)\right], \\
& \hat{u}_{J 1}^{ \pm}=A M_{+}\left(x_{N}\right) \sum_{\ell=1}^{N}\left[R_{J \ell,-1}^{ \pm} \hat{h}_{\ell}(0)+R_{J \ell, 0}^{ \pm} \hat{k}_{\ell}(0)\right], \\
& \hat{u}_{J 2}^{ \pm}=A e^{\mp B_{ \pm} x_{N}} \sum_{\ell=1}^{N}\left[S_{J \ell,-2}^{ \pm} \hat{h}_{\ell}(0)+S_{J \ell,-1}^{ \pm} \hat{k}_{\ell}(0)\right], \\
& \hat{u}_{J 3}^{ \pm}=e^{\mp B_{ \pm} x_{N}}\left[T_{J,-1}^{ \pm} \hat{h}_{J}(0)+T_{J, 0}^{ \pm} \hat{k}_{J J}(0)\right], \quad \hat{u}_{j 4}^{+}=0, \\
& \hat{u}_{N 4}^{+}=A_{+} M_{+}\left(x_{N}\right) U_{N, 0}^{+} \hat{k}_{N}(0)
\end{aligned}
$$

with

$$
\begin{aligned}
& R_{J \ell,-1}^{ \pm} \in \mathbf{M}_{-1,2}, \quad R_{J \ell, 0}^{ \pm} \in \mathbf{M}_{0,2}, \quad S_{J \ell,-2}^{ \pm} \in \mathbf{M}_{-2,2}, \quad S_{J \ell,-1}^{ \pm} \in \mathbf{M}_{-1,2}, \\
& T_{J,-1}^{ \pm} \in \mathbf{M}_{-1,1}, \quad T_{J, 0}^{ \pm} \in \mathbf{M}_{0,1}, \quad U_{N, 0}^{+} \in \mathbf{M}_{0,1}, \quad p_{\ell, 0}^{-} \in \mathbf{M}_{0,2}, \quad p_{\ell, 1}^{-} \in \mathbf{M}_{1,2} .
\end{aligned}
$$


Here and in the following, $M_{ \pm}\left(x_{N}\right)$ denote the Stokes kernels defined by

$$
M_{+}\left(x_{N}\right)=\frac{e^{-B_{+} x_{N}}-e^{-A_{+} x_{N}}}{B_{+}-A_{+}}, \quad M_{-}\left(x_{N}\right)=\frac{e^{B_{-} x_{N}}-e^{A x_{N}}}{B_{-}-A} .
$$

From now on, we prove (2.7). We find solutions $\hat{u}_{ \pm J}$ to problem (2.2)-(2.3) of the forms

$$
\begin{aligned}
& \hat{u}_{+J}=\alpha_{+J}\left(e^{-B_{+} x_{N}}-e^{-A_{+} x_{N}}\right)+\beta_{+J} e^{-B_{+} x_{N}}, \\
& \hat{u}_{-J}=\alpha_{-J}\left(e^{B_{-} x_{N}}-e^{A x_{N}}\right)+\beta_{-J} e^{B_{-} x_{N}}, \quad \hat{p}_{-}=\gamma_{-} e^{A x_{N}} .
\end{aligned}
$$

Using the symbols $B_{ \pm}$, we write (2.2) as follows:

$$
\begin{cases}\mu_{+} B_{+}^{2} \hat{u}_{+j}-\mu_{+} D_{N}^{2} \hat{u}_{+j}-\left(v_{+}+\delta\right) i \xi_{j}\left(i \xi^{\prime} \cdot \hat{u}_{+}^{\prime}+D_{N} \hat{u}_{+N}\right)=0 & \left(x_{N}>0\right), \\ \mu_{+} B_{+}^{2} \hat{u}_{+N}-\mu_{+} D_{N}^{2} \hat{u}_{+N}-\left(v_{+}+\delta\right) D_{N}\left(i \xi^{\prime} \cdot \hat{u}_{+}^{\prime}+D_{N} \hat{u}_{+N}\right)=0 & \left(x_{N}>0\right), \\ \mu_{-} B_{-}^{2} \hat{u}_{-j}-\mu_{-} D_{N}^{2} \hat{u}_{-j}+i \xi_{j} \hat{p}_{-}=0 & \left(x_{N}<0\right), \\ \mu_{-} B_{-}^{2} \hat{u}_{-N}-\mu_{-} D_{N}^{2} \hat{u}_{-N}+D_{N} \hat{p}_{-}=0 & \left(x_{N}<0\right), \\ i \xi^{\prime} \cdot \hat{u}_{-}^{\prime}+D_{N} \hat{u}_{-N}=0 & \left(x_{N}<0\right) .\end{cases}
$$

Substituting the formulas of $u_{ \pm J}$ in (2.10) and (2.11) and equating the coefficients of $e^{\mp B_{ \pm} x_{N}}$, $e^{-A_{+} x_{N}}$, and $e^{A x_{N}}$, we have

$$
\begin{aligned}
& \mu_{+}\left(A_{+}^{2}-B_{+}^{2}\right) \alpha_{+j}+\left(v_{+}+\delta\right) i \xi_{j}\left(i \xi^{\prime} \cdot \alpha_{+}^{\prime}-A_{+} \alpha_{+N}\right)=0, \\
& \mu_{+}\left(A_{+}^{2}-B_{+}^{2}\right) \alpha_{+N}-\left(v_{+}+\delta\right) A_{+}\left(i \xi^{\prime} \cdot \alpha_{+}^{\prime}-A_{+} \alpha_{+N}\right)=0, \\
& i \xi^{\prime} \cdot \alpha_{+}^{\prime}-\alpha_{+N} B_{+}+i \xi^{\prime} \cdot \beta_{+}^{\prime}-\beta_{+N} B_{+}=0, \\
& \mu_{-}\left(A^{2}-B_{-}^{2}\right) \alpha_{-j}+i \xi_{j} \gamma_{-}=0, \quad \mu_{-}\left(A^{2}-B_{-}^{2}\right) \alpha_{-N}+A \gamma_{-}=0, \\
& i \xi^{\prime} \cdot \alpha_{-}^{\prime}+\alpha_{-N} B_{-}+i \xi^{\prime} \cdot \beta_{-}^{\prime}+\beta_{-N} B_{-}=0, \quad i \xi^{\prime} \cdot \alpha_{-}^{\prime}+A \alpha_{-N}=0 .
\end{aligned}
$$

First, we represent $i \xi^{\prime} \cdot \alpha_{ \pm}^{\prime}, \alpha_{ \pm N}$ and $\gamma_{-}$by $i \xi^{\prime} \cdot \beta_{ \pm}^{\prime}$ and $\beta_{+N}$. Namely, it follows from (2.12) that

$$
\begin{aligned}
& i \xi^{\prime} \cdot \alpha_{+}^{\prime}=\frac{A^{2}}{A_{+} B_{+}-A^{2}}\left(i \xi^{\prime} \cdot \beta_{+}^{\prime}-B_{+} \beta_{+N}\right), \quad \alpha_{+N}=\frac{A_{+}}{A_{+} B_{+}-A^{2}}\left(i \xi^{\prime} \cdot \beta_{+}^{\prime}-B_{+} \beta_{+N}\right) \\
& i \xi^{\prime} \cdot \alpha_{-}^{\prime}=\frac{A}{B_{-}-A}\left(i \xi^{\prime} \cdot \beta_{-}^{\prime}+B_{-} \beta_{-N}\right), \quad \alpha_{-N}=\frac{-1}{B_{-}-A}\left(i \xi^{\prime} \cdot \beta_{-}^{\prime}+B_{-} \beta_{-N}\right) \\
& \gamma_{-}=-\frac{\mu_{-}\left(A+B_{-}\right)}{A}\left(i \xi^{\prime} \cdot \beta_{-}^{\prime}+B_{-} \beta_{-N}\right) .
\end{aligned}
$$

Substituting the relations

$$
\hat{u}_{ \pm J}(0)=\beta_{ \pm J}, \quad \partial_{N} \hat{u}_{+J}(0)=\left(A_{+}-B_{+}\right) \alpha_{+J}-B_{+} \beta_{+J}, \quad \partial_{N} \hat{u}_{-J}(0)=\left(B_{-}-A\right) \alpha_{-J}+B_{-} \beta_{-J}
$$

into (2.3), we have

$$
\begin{aligned}
& \beta_{+J}=\beta_{-J}+\hat{k}_{J}(0), \\
& \mu_{+}\left(\left(B_{+}-A_{+}\right) \alpha_{+j}+B_{+} \beta_{+j}-i \xi_{j} \beta_{+N}\right)+\mu_{-}\left(\left(B_{-}-A\right) \alpha_{-j}+B_{-} \beta_{-j}+i \xi_{j} \beta_{-N}\right)=-\hat{h}_{j}(0),
\end{aligned}
$$




$$
\begin{aligned}
& 2 \mu_{+}\left(\left(B_{+}-A_{+}\right) \alpha_{+N}+B_{+} \beta_{+N}\right) \\
& \quad+\left(v_{+}-\mu_{+}+\delta\right)\left(-i \xi^{\prime} \cdot \beta_{+}^{\prime}+\left(B_{+}-A_{+}\right) \alpha_{+N}+B_{+} \beta_{+N}\right) \\
& \quad+2 \mu_{-}\left(\left(B_{-}-A\right) \alpha_{-N}+B_{-} \beta_{-N}\right)-\gamma_{-}=-\hat{h}_{N}(0) .
\end{aligned}
$$

Using (2.14) and (2.13), we have

$$
\begin{aligned}
& -i \xi^{\prime} \cdot \hat{h}^{\prime}(0)=L_{11}^{+}\left(i \xi^{\prime} \cdot \beta_{+}^{\prime}\right)+L_{11}^{-}\left(i \xi^{\prime} \cdot \beta_{-}^{\prime}\right)+L_{12}^{+} A \beta_{+N}+L_{12}^{-} A \beta_{-N}, \\
& -A \hat{h}_{N}(0)=L_{21}^{+}\left(i \xi^{\prime} \cdot \beta_{+}^{\prime}\right)+L_{21}^{-}\left(i \xi^{\prime} \cdot \beta_{-}^{\prime}\right)+L_{22}^{+} A \beta_{+N}+L_{22}^{-} \beta_{-N}
\end{aligned}
$$

with

$$
\begin{aligned}
& L_{11}^{+}=\mu_{+} \frac{A_{+}\left(B_{+}^{2}-A^{2}\right)}{A_{+} B_{+}-A^{2}}, \quad L_{11}^{-}=\mu_{-}\left(A+B_{-}\right), \\
& L_{12}^{+}=\mu_{+} \frac{A\left(2 A_{+} B_{+}-A^{2}-B_{+}^{2}\right)}{A_{+} B_{+}-A^{2}}, \quad L_{12}^{-}=\mu_{-}\left(B_{-}-A\right), \\
& L_{21}^{+}=A\left\{2 \mu_{+} \frac{A_{+}\left(B_{+}-A_{+}\right)}{A_{+} B_{+}-A^{2}}-\left(v_{+}-\mu_{+}+\delta\right) \frac{A_{+}^{2}-A^{2}}{A_{+} B_{+}-A^{2}}\right\}, \quad L_{21}^{-}=\mu_{-}\left(B_{-}-A\right), \\
& L_{22}^{+}=\left(\mu_{+}+v_{+}+\delta\right) \frac{B_{+}\left(A_{+}^{2}-A^{2}\right)}{A_{+} B_{+}-A^{2}}, \quad L_{22}^{-}=\mu_{-}\left(A+B_{-}\right) B_{-} .
\end{aligned}
$$

As is seen in Section 4, we have

$$
\begin{array}{lll}
L_{11}^{+} \in \mathbf{M}_{1,1}, & L_{11}^{-} \in \mathbf{M}_{1,2}, & L_{12}^{ \pm} \in \mathbf{M}_{1,2}, \\
L_{21}^{ \pm} \in \mathbf{M}_{1,2}, & L_{22}^{+} \in \mathbf{M}_{1,1}, & L_{22}^{-} \in \mathbf{M}_{2,2} .
\end{array}
$$

Noting the relation $\beta_{+J}=\beta_{-J}+\hat{k}_{J}(0)$, and setting

$$
\begin{aligned}
& L_{11}=L_{11}^{+}+L_{11}^{-}, \quad L_{12}=L_{12}^{+}+L_{12}^{-}, \quad L_{21}=L_{21}^{+}+L_{21}^{-}, \\
& L_{22}=L_{22}^{+} A+L_{22}^{-}, \quad L=\left(\begin{array}{cc}
L_{11} & A L_{12} \\
L_{21} & L_{22}
\end{array}\right),
\end{aligned}
$$

we have

$$
L\left(\begin{array}{c}
i \xi^{\prime} \cdot \beta_{-}^{\prime} \\
\beta_{-N}
\end{array}\right)=\left(\begin{array}{c}
\hat{H}^{\prime}(0) \\
\hat{H}_{N}(0)
\end{array}\right)
$$

with

$$
\begin{aligned}
& \hat{H}^{\prime}(0)=-i \xi^{\prime} \cdot \hat{h}^{\prime}(0)-L_{11}^{+} i \xi^{\prime} \cdot \hat{k}^{\prime}(0)-L_{12}^{+} A \hat{k}_{N}(0), \\
& \hat{H}_{N}(0)=-A \hat{h}_{N}(0)-L_{21}^{+} i \xi^{\prime} \cdot \hat{k}^{\prime}(0)-A L_{22}^{+} \hat{k}_{N}(0) .
\end{aligned}
$$

By Lemma 2.2 and (2.16), we see that

$$
L_{11} \in \mathbf{M}_{1,2}, \quad L_{12} \in \mathbf{M}_{1,2}, \quad L_{21} \in \mathbf{M}_{1,2}, \quad L_{22} \in \mathbf{M}_{2,2} .
$$


The most important fact of this paper is that $\operatorname{det} L \neq 0$ for any $\left(\lambda, \xi^{\prime}\right) \in \tilde{\Gamma}_{\epsilon, \lambda_{0}}$ and

$$
(\operatorname{det} L)^{-1} \in \mathbf{M}_{-3,2} \text {. }
$$

This fact is proved in Section 5, which is the highlight of this paper. Since

$$
L^{-1}=\frac{1}{\operatorname{det} L}\left(\begin{array}{cc}
L_{22} & -A L_{12} \\
-L_{21} & L_{11}
\end{array}\right),
$$

we have

$$
\begin{aligned}
i \xi^{\prime} \cdot \beta_{-}^{\prime}= & \frac{1}{\operatorname{det} L}\left(L_{22} \hat{H}^{\prime}(0)-A L_{12} \hat{H}_{N}(0)\right) \\
= & \frac{1}{\operatorname{det} L}\left(-L_{22} i \xi \cdot \hat{h}^{\prime}(0)+A^{2} L_{12} \hat{h}_{N}(0)+\left(A L_{12} L_{21}^{+}-L_{11}^{+} L_{22}\right) i \xi^{\prime} \cdot \hat{k}^{\prime}(0)\right. \\
& \left.\quad+\left(A L_{12} L_{22}^{+}-L_{12}^{+} L_{22}\right) A \hat{k}_{N}(0)\right), \\
\beta_{-N}= & \frac{1}{\operatorname{det} L}\left(L_{11} \hat{H}_{N}(0)-L_{21} \hat{H}^{\prime}(0)\right) \\
= & \frac{1}{\operatorname{det} L}\left(L_{21} i \xi^{\prime} \cdot \hat{h}^{\prime}(0)-L_{11} A \hat{h}_{N}(0)+\left(L_{11}^{+} L_{21}-L_{11} L_{21}^{+}\right) i \xi^{\prime} \cdot \hat{k}^{\prime}(0)\right. \\
& \left.+\left(L_{12}^{+} L_{21}-L_{11} L_{22}^{+}\right) A \hat{k}_{N}(0)\right) .
\end{aligned}
$$

Writing $i \xi^{\prime} \cdot \hat{k}^{\prime}(0)=A \sum_{\ell=1}^{N-1} \frac{i \xi_{\ell}}{A} \hat{k}_{\ell}(0)$ and using the relations $\beta_{+J}=\beta_{-J}+\hat{k}_{J}(0)$, by (2.21), we have

$$
\begin{aligned}
& i \xi^{\prime} \cdot \beta_{+}^{\prime}-B_{+} \beta_{+N}=-B_{+} \hat{k}_{N}(0)+\sum_{\ell=1}^{N} A\left(P_{\ell,-1}^{+} \hat{h}_{\ell}(0)+P_{\ell, 0}^{+} \hat{k}_{\ell}(0)\right), \\
& i \xi^{\prime} \cdot \beta_{-}^{\prime}+B_{-} \beta_{-N}=\sum_{\ell=1}^{N} A\left(P_{\ell,-1}^{-} \hat{h}_{\ell}(0)+P_{\ell, 0}^{-} \hat{k}_{\ell}(0)\right)
\end{aligned}
$$

with

$$
\begin{aligned}
& P_{\ell,-1}^{+}=\frac{-\left(L_{22}+B_{+} L_{21}\right) i \xi_{\ell}}{A \operatorname{det} L}, \quad P_{N,-1}^{+}=\frac{A L_{12}+B_{+} L_{11}}{\operatorname{det} L}, \\
& P_{\ell, 0}^{+}=\frac{\left(A L_{12} L_{21}^{+}-L_{22} L_{11}^{+}-B_{+}\left(L_{11}^{+} L_{21}-L_{11} L_{21}^{+}\right)\right) i \xi_{\ell}}{A \operatorname{det} L}+\frac{i \xi_{\ell}}{A}, \\
& P_{N, 0}^{+}=\frac{A L_{12} L_{22}^{+}-L_{12}^{+} L_{22}-B_{+}\left(L_{12}^{+} L_{21}-L_{11} L_{22}^{+}\right)}{\operatorname{det} L}, \\
& P_{\ell,-1}^{-}=\frac{-\left(L_{22}-B_{-} L_{21}\right) i \xi_{\ell}}{A \operatorname{det} L}, \quad P_{N,-1}^{-}=\frac{A L_{12}-B_{-} L_{11}}{\operatorname{det} L}, \\
& P_{\ell, 0}^{-}=\frac{\left(A L_{12} L_{21}^{+}-L_{22} L_{11}^{+}+B_{-}\left(L_{11}^{+} L_{21}-L_{11} L_{21}^{+}\right)\right) i \xi_{\ell}}{A \operatorname{det} L}, \\
& P_{N, 0}^{-}=\frac{A L_{12} L_{22}^{+}-L_{12}^{+} L_{22}+B_{-}\left(L_{12}^{+} L_{21}-L_{11} L_{22}^{+}\right)}{\operatorname{det} L}
\end{aligned}
$$

for $\ell=1, \ldots, N-1$. By Lemma 2.2, (2.16), (2.19), and (2.20), we have

$$
P_{J,-1}^{ \pm} \in \mathbf{M}_{-1,2}, \quad P_{J, 0}^{ \pm} \in \mathbf{M}_{0,2} .
$$


By (2.13) we have

$$
\begin{aligned}
\hat{p}_{-}\left(x_{N}\right) & =-\mu_{-} \frac{\left(A+B_{-}\right)}{A}\left(i \xi^{\prime} \cdot \beta_{-}^{\prime}+B_{-} \beta_{-N}\right) e^{A x_{N}} \\
& =-\mu_{-}\left(A+B_{-}\right) \sum_{\ell=1}^{N}\left(P_{\ell,-1}^{-} \hat{h}_{\ell}(0)+P_{\ell, 0}^{-} \hat{k}_{\ell}(0)\right) e^{A x_{N}},
\end{aligned}
$$

so that setting $p_{\ell, 0}^{-}=-\mu_{-}\left(A+B_{-}\right) P_{\ell,-1}^{-}$and $p_{\ell, 1}^{-}=-\mu_{-}\left(A+B_{-}\right) P_{\ell, 0}^{-}$, we have the formula of $\hat{p}_{-}\left(x_{N}\right)$ in $(2.7)$.

By (2.12), we have

$$
\begin{aligned}
& \left(B_{+}-A_{+}\right) \alpha_{+j}=\frac{\left(v_{+}+\delta\right) i \xi_{j}}{\mu_{+}\left(A_{+}+B_{+}\right)}\left(i \xi^{\prime} \cdot \alpha_{+}^{\prime}-A_{+} \alpha_{+N}\right), \\
& \left(B_{+}-A_{+}\right) \alpha_{+N}=-\frac{\left(\nu_{+}+\delta\right) A_{+}}{\mu_{+}\left(A_{+}+B_{+}\right)}\left(i \xi^{\prime} \cdot \alpha_{+}^{\prime}-A_{+} \alpha_{+N}\right), \\
& \left(B_{-}-A\right) \alpha_{-j}=-\frac{i \xi_{j}}{A}\left(i \xi^{\prime} \cdot \beta^{\prime}+B_{-} \beta_{-N}\right), \quad\left(B_{-}-A\right) \alpha_{-N}=-\left(i \xi^{\prime} \cdot \beta^{\prime}+B_{-} \beta_{-N}\right) .
\end{aligned}
$$

Since $i \xi^{\prime} \cdot \alpha_{+}^{\prime}-A_{+} \alpha_{+N}=\frac{A^{2}-A_{+}^{2}}{A_{+} B_{+}-A^{2}}\left(i \xi^{\prime} \cdot \beta_{+}^{\prime}-B_{+} \beta_{-N}\right)$ as follows from (2.13), by (2.22) we have

$$
\begin{aligned}
\left(B_{+}-A_{+}\right) \alpha_{+j}=- & \frac{\left(v_{+}+\delta\right)\left(i \xi_{j}\right) B_{+}}{\mu_{+}\left(A_{+}+B_{+}\right)} \frac{A^{2}-A_{+}^{2}}{A_{+} B_{+}-A^{2}} \hat{k}_{N}(0) \\
& +\frac{\left(v_{+}+\delta\right) i \xi_{j}}{\mu_{+}\left(A_{+}+B_{+}\right)} \frac{A^{2}-A_{+}^{2}}{A_{+} B_{+}-A^{2}} A \sum_{\ell=1}^{N}\left(P_{\ell,-1}^{+} \hat{h}_{\ell}(0)+P_{\ell, 0}^{+} \hat{k}_{\ell}(0)\right), \\
\left(B_{+}-A_{+}\right) \alpha_{+N}= & \frac{\left(v_{+}+\delta\right) A_{+} B_{+}}{\mu_{+}\left(A_{+}+B_{+}\right)} \frac{A^{2}-A_{+}^{2}}{A_{+} B_{+}-A^{2}} \hat{k}_{N}(0) \\
& -\frac{\left(v_{+}+\delta\right) A_{+}}{\mu_{+}\left(A_{+}+B_{+}\right)} \frac{A^{2}-A_{+}^{2}}{A_{+} B_{+}-A^{2}} A \sum_{\ell=1}^{N}\left(P_{\ell,-1}^{+} \hat{h}_{\ell}(0)+P_{\ell, 0}^{+} \hat{k}_{\ell}(0)\right), \\
\left(B_{-}-A\right) \alpha_{-j}=- & \frac{i \xi_{j}}{A} \sum_{\ell=1}^{N}\left(P_{\ell,-1}^{-} \hat{h}_{\ell}(0)+P_{\ell, 0}^{-} \hat{k}_{\ell}(0)\right), \\
\left(B_{-}-A\right) \alpha_{-N}= & -A \sum_{\ell=1}^{N}\left(P_{\ell,-1}^{-} \hat{h}_{\ell}(0)+P_{\ell, 0}^{-} \hat{k}_{\ell}(0)\right)
\end{aligned}
$$

for $j=1, \ldots, N-1$. By (2.24) we have

$$
\begin{aligned}
\left(B_{+}-A_{+}\right) \alpha_{+j}= & A\left[\sum_{\ell=1}^{N} \frac{\left(v_{+}+\delta\right)\left(i \xi_{j}\right) P_{\ell,-1}^{+}}{\mu_{+}\left(A_{+}+B_{+}\right)} \frac{A^{2}-A_{+}^{2}}{A_{+} B_{+}-A^{2}} \hat{h}_{\ell}(0)\right. \\
& +\sum_{\ell=1}^{N-1} \frac{\left(v_{+}+\delta\right)\left(i \xi_{j}\right) P_{\ell, 0}^{+}}{\mu_{+}\left(A_{+}+B_{+}\right)} \frac{A^{2}-A_{+}^{2}}{A_{+} B_{+}-A^{2}} \hat{k}_{\ell}(0) \\
& \left.+\frac{\left(v_{+}+\delta\right)}{\mu_{+}\left(A_{+}+B_{+}\right)} \frac{A^{2}-A_{+}^{2}}{A_{+} B_{+}-A^{2}}\left(-\frac{i \xi_{j}}{A} B_{+}+i \xi_{j} P_{N, 0}^{+}\right) \hat{k}_{N}(0)\right], \\
\left(B_{+}-A_{+}\right) \alpha_{+N}= & \frac{\left(v_{+}+\delta\right) A_{+} B_{+}}{\mu_{+}\left(A_{+}+B_{+}\right)} \frac{A^{2}-A_{+}^{2}}{A_{+} B_{+}-A^{2}} \hat{k}_{N}(0)
\end{aligned}
$$




$$
\begin{aligned}
& -A\left[\sum_{\ell=1}^{N} \frac{\left(v_{+}+\delta\right) A_{+} P_{\ell,-1}^{+}}{\mu_{+}\left(A_{+}+B_{+}\right)} \frac{A^{2}-A_{+}^{2}}{A_{+} B_{+}-A^{2}} \hat{h}_{\ell}(0)\right. \\
& \left.+\sum_{\ell=1}^{N} \frac{\left(v_{+}+\delta\right) A_{+} P_{\ell, 0}^{+}}{\mu_{+}\left(A_{+}+B_{+}\right)} \frac{A^{2}-A_{+}^{2}}{A_{+} B_{+}-A^{2}} \hat{k}_{\ell}(0)\right] .
\end{aligned}
$$

Since $\left(e^{-B_{+} x_{N}}-e^{-A_{+} x_{N}}\right) \alpha_{+J}=M_{+}\left(x_{N}\right)\left(B_{+}-A_{+}\right) \alpha_{+J}$, setting

$$
\begin{aligned}
& R_{j \ell,-1}^{+}=\frac{\left(v_{+}+\delta\right)\left(i \xi_{j}\right) P_{\ell,-1}^{+}}{\mu_{+}\left(A_{+}+B_{+}\right)} \frac{A^{2}-A_{+}^{2}}{A_{+} B_{+}-A^{2}}, \quad R_{j \ell^{\prime}, 0}^{+}=\frac{\left(v_{+}+\delta\right)\left(i \xi_{j}\right) P_{\ell^{\prime}, 0}^{+}}{\mu_{+}\left(A_{+}+B_{+}\right)} \frac{A^{2}-A_{+}^{2}}{A_{+} B_{+}-A^{2}}, \\
& R_{j N, 0}^{+}=\frac{\left(v_{+}+\delta\right)}{\mu_{+}\left(A_{+}+B_{+}\right)} \frac{A^{2}-A_{+}^{2}}{A_{+} B_{+}-A^{2}}\left(-\frac{i \xi_{j}}{A} B_{+}+i \xi_{j} P_{N, 0}^{+}\right), \\
& R_{N \ell,-1}^{+}=-\frac{\left(v_{+}+\delta\right) A_{+} P_{\ell,-1}^{+}}{\mu_{+}\left(A_{+}+B_{+}\right)} \frac{A^{2}-A_{+}^{2}}{A_{+} B_{+}-A^{2}}, \quad R_{N \ell, 0}^{+}=-\frac{\left(v_{+}+\delta\right) A_{+} P_{\ell, 0}^{+}}{\mu_{+}\left(A_{+}+B_{+}\right)} \frac{A^{2}-A_{+}^{2}}{A_{+} B_{+}-A^{2}}, \\
& U_{N, 0}^{+}=\frac{\left(v_{+}+\delta\right) B_{+}}{\mu_{+}\left(A_{+}+B_{+}\right)} \frac{A^{2}-A_{+}^{2}}{A_{+} B_{+}-A^{2}}
\end{aligned}
$$

for $\ell=1, \ldots, N$ and $j, \ell^{\prime}=1, \ldots, N-1$, we have $\hat{u}_{j 1}^{+}$and $\hat{u}_{N 4}^{+}$in (2.7). As is seen in Section 4 below, we have

$$
A_{+} \in \mathbf{M}_{1,1}, \quad B_{+} \in \mathbf{M}_{1,1}, \quad\left(A_{+}+B_{+}\right)^{-1} \in \mathbf{M}_{-1,1}, \quad \frac{A^{2}-A_{+}^{2}}{A_{+} B_{+}-A^{2}} \in \mathbf{M}_{0,1},
$$

which, combined with (2.23), furnishes $R_{J \ell,-1}^{+} \in \mathbf{M}_{-1,2}, R_{J \ell, 0}^{+} \in \mathbf{M}_{0,2}$, and $U_{N, 0}^{+} \in \mathbf{M}_{0,1}$.

Analogously, in view of (2.24) we set

$$
R_{j \ell,-1}^{-}=-\frac{i \xi_{j}}{A} P_{\ell,-1}^{-}, \quad R_{j \ell, 0}^{-}=-\frac{i \xi_{j}}{A} P_{\ell, 0}^{-}, \quad R_{N \ell,-1}^{-}=-P_{\ell,-1}^{-}, \quad R_{N \ell, 0}^{-}=-P_{\ell, 0}^{-}
$$

for $\ell=1, \ldots, N$, and $j=1, \ldots, N-1$, we have $u_{J 1}^{-}\left(x_{N}\right)$ in (2.7). By (2.23) and (2.25), we have $R_{J \ell,-1}^{-} \in \mathbf{M}_{-1,2}$ and $R_{J \ell, 0}^{-} \in \mathbf{M}_{0,2}$.

Using (2.21), we represent $\beta_{-N}$ by

$$
\beta_{-N}=A \sum_{\ell=1}^{N}\left(Q_{\ell,-2} \hat{h}_{\ell}(0)+Q_{\ell,-1} \hat{k}_{\ell}(0)\right)
$$

with

$$
\begin{aligned}
Q_{\ell,-2} & =\frac{L_{21} i \xi_{\ell}}{A \operatorname{det} L}, \quad Q_{N,-2}=-\frac{L_{11}}{\operatorname{det} L}, \\
Q_{\ell,-1} & =\frac{\left(L_{11}^{+} L_{21}-L_{11} L_{21}^{+}\right) i \xi_{\ell}}{A \operatorname{det} L}, \quad Q_{N,-1}=\frac{\left(L_{12}^{+} L_{21}-L_{11} L_{22}^{+}\right)}{\operatorname{det} L}
\end{aligned}
$$

for $\ell=1, \ldots, N-1$. By Lemma 2.2, (2.16), (2.19), and (2.20), we have

$$
Q_{J,-2} \in \mathbf{M}_{-2,2}, \quad Q_{J,-1} \in \mathbf{M}_{-1,2} .
$$

In particular, noting that $\beta_{+N}=\hat{k}_{N}(0)+\beta_{-N}$ and setting $S_{N \ell,-2}^{ \pm}=Q_{\ell,-2}, S_{N \ell,-1}^{ \pm}=Q_{\ell,-1}(\ell=$ $1, \ldots, N), T_{N,-1}^{ \pm}=0, T_{N, 0}^{+}=1$ and $T_{N, 0}^{-}=0$, we have the $\hat{u}_{N 2}^{ \pm}$and $\hat{u}_{N 3}^{ \pm}$in (2.7), and by (2.27) $S_{N \ell,-2}^{ \pm} \in \mathbf{M}_{-2,2}, S_{N \ell,-1}^{ \pm} \in \mathbf{M}_{-1,2}, T_{N,-1}^{ \pm} \in \mathbf{M}_{-1,1}$, and $T_{N, 0}^{ \pm} \in \mathbf{M}_{0,1}$ for $\ell=1, \ldots, N$. 
From (2.14) it follows that

$$
\begin{aligned}
-\hat{h}_{j}(0)= & \mu_{+} B_{+} \beta_{+j}+\mu_{-} B_{-} \beta_{-j}+\mu_{+}\left(B_{+}-A_{+}\right) \alpha_{+j} \\
& +\mu_{-}\left(B_{-}-A\right) \alpha_{-j}-\left(\mu_{+} \beta_{+N}-\mu_{-} \beta_{-N}\right) i \xi_{j} .
\end{aligned}
$$

Noting that $\beta_{+J}=\beta_{-J}+\hat{k}_{J}(0)$, we have

$$
\begin{aligned}
\beta_{ \pm j}= & \frac{-1}{\mu_{+} B_{+}+\mu_{-} B_{-}} \hat{h}_{j}(0) \pm \frac{\mu_{\mp} B_{\mp}}{\mu_{+} B_{+}+\mu_{-} B_{-}} \hat{k}_{j}(0)+\frac{\mu_{+} i \xi_{j}}{\mu_{+} B_{+}+\mu_{-} B_{-}} \hat{k}_{N}(0) \\
& -\frac{\mu_{+}\left(B_{+}-A_{+}\right)}{\mu_{+} B_{+}+\mu_{-} B_{-}} \alpha_{+j}-\frac{\mu_{-}\left(B_{-}-A\right)}{\mu_{+} B_{+}+\mu_{-} B_{-}} \alpha_{-j}+\frac{\left(\mu_{+}-\mu_{-}\right) i \xi_{j}}{\mu_{+} B_{+}+\mu_{-} B_{-}} \beta_{-N},
\end{aligned}
$$

which, combined with (2.24) and (2.26), furnishes

$$
\begin{aligned}
\beta_{ \pm j}= & \frac{-1}{\mu_{+} B_{+}+\mu_{-} B_{-}} \hat{h}_{j}(0) \pm \frac{\mu_{\mp} B_{\mp}}{\mu_{+} B_{+}+\mu_{-} B_{-}} \hat{k}_{j}(0)+\frac{\mu_{+} i \xi_{j}}{\mu_{+} B_{+}+\mu_{-} B_{-}} \hat{k}_{N}(0) \\
& +\frac{\left(v_{+}+\delta\right) i \xi_{j} B_{+}}{\left(\mu_{+} B_{+}+\mu_{-} B_{-}\right)\left(B_{+}+A_{+}\right)} \frac{A^{2}-A_{+}^{2}}{A_{+} B_{+}-A^{2}} \hat{k}_{N}(0) \\
& -\frac{\left(\nu_{+}+\delta\right) i \xi_{j}}{\left(\mu_{+} B_{+}+\mu_{-} B_{-}\right)\left(B_{+}+A_{+}\right)} \frac{A^{2}-A_{+}^{2}}{A_{+} B_{+}-A^{2}} A \sum_{\ell=1}^{N}\left(P_{\ell,-1}^{+} \hat{h}_{\ell}(0)+P_{\ell, 0}^{+} \hat{k}_{\ell}(0)\right) \\
& +\frac{\mu_{-} i \xi_{j}}{\left(\mu_{+} B_{+}+\mu_{-} B_{-}\right) A} A \sum_{\ell=1}^{N}\left(P_{\ell,-1}^{-} \hat{h}_{\ell}(0)+P_{\ell, 0}^{-} \hat{k}_{\ell}(0)\right) \\
& +\frac{\left(\mu_{+}-\mu_{-}\right) i \xi_{j}}{\left(\mu_{+} B_{+}+\mu_{-} B_{-}\right)} A \sum_{\ell=1}^{N}\left(Q_{\ell,-2} \hat{h}_{\ell}(0)+Q_{\ell,-1} \hat{k}_{\ell}(0)\right) .
\end{aligned}
$$

Thus, we set

$$
\begin{aligned}
S_{j \ell,-2}^{ \pm}= & -\frac{\left(v_{+}+\delta\right)\left(i \xi_{j}\right) P_{\ell,-1}^{+}}{\left(\mu_{+} B_{+}+\mu_{-} B_{-}\right)\left(A_{+}+B_{+}\right)} \frac{A^{2}-A_{+}^{2}}{A_{+} B_{+}-A^{2}} \\
& +\frac{\mu_{-}\left(i \xi_{j}\right) P_{\ell,-1}^{-}}{\left(\mu_{+} B_{+}+\mu_{-} B_{-}\right) A}+\frac{\left(\mu_{+}-\mu_{-}\right)\left(i \xi_{j}\right) Q_{\ell,-2}}{\mu_{+} B_{+}+\mu_{-} B_{-}}, \\
S_{j \ell^{\prime},-1}^{ \pm}= & -\frac{\left(v_{+}+\delta\right)\left(i \xi_{j}\right) P_{\ell^{\prime}, 0}^{+}}{\left(\mu_{+} B_{+}+\mu_{-} B_{-}\right)\left(A_{+}+B_{+}\right)} \frac{A^{2}-A_{+}^{2}}{A_{+} B_{+}-A^{2}} \\
& +\frac{\mu_{-}\left(i \xi_{j}\right) P_{\ell^{\prime}, 0}^{-}}{\left(\mu_{+} B_{+}+\mu_{-} B_{-}\right) A}+\frac{\left(\mu_{+}-\mu_{-}\right)\left(i \xi_{j}\right) Q_{\ell^{\prime},-1}}{\mu_{+} B_{+}+\mu_{-} B_{-}}, \\
S_{j N,-1}^{ \pm}= & \frac{\mu_{+} i \xi_{j}}{\left(\mu_{+} B_{+}+\mu_{-} B_{-}\right) A}+\frac{\left(\nu_{+}+\delta\right)\left(i \xi_{j}\right) B_{+}}{\left(\mu_{+} B_{+}+\mu_{-} B_{-}\right)\left(A_{+}+B_{+}\right) A} \frac{A^{2}-A_{+}^{2}}{A_{+} B_{+}-A^{2}} \\
& -\frac{\left(v_{+}+\delta\right)\left(i \xi_{j}\right) P_{N, 0}^{+}}{\left(\mu_{+} B_{+}+\mu_{-} B_{-}\right)\left(A_{+}+B_{+}\right)} \frac{A^{2}-A_{+}^{2}}{A_{+} B_{+}-A^{2}} \\
& +\frac{\mu_{-}\left(i \xi_{j}\right) P_{N, 0}^{-}}{\left(\mu_{+} B_{+}+\mu_{-} B_{-}\right) A}+\frac{\left(\mu_{+}-\mu_{-}\right)\left(i \xi_{j}\right) Q_{N,-1}}{\mu_{+} B_{+}+\mu_{-} B_{-}}, \\
T_{j,-1}^{ \pm}= & \frac{-1}{\mu_{+} B_{+}+\mu_{-} B_{-}}, \quad T_{j, 0}^{+}=\frac{\mu_{-} B_{-}}{\mu_{+} B_{+}+\mu_{-} B_{-}}, \quad T_{j, 0}^{-}=-\frac{\mu_{+} B_{+}}{\mu_{+} B_{+}+\mu_{-} B_{-}},
\end{aligned}
$$


so that we have the $\hat{u}_{j 2}^{ \pm}$and $\hat{u}_{j 3}^{ \pm}$in (2.7). Moreover, as is seen in Section 4, we have

$$
\left(\mu_{+} B_{+}+\mu_{-} B_{-}\right)^{-1} \in \mathbf{M}_{-1,1}
$$

so that by (2.23), (2.25), (2.27), and (2.29) we have $S_{j \ell,-2}^{ \pm} \in \mathbf{M}_{-2,2}, S_{j \ell,-1}^{ \pm} \in \mathbf{M}_{-1,2}, T_{j,-1}^{ \pm} \in \mathbf{M}_{-1,1}$, and $T_{j, 0}^{ \pm} \in \mathbf{M}_{0,1}$. This completes the proof of (2.7).

To construct our solution operator from the solution formulas in (2.7), first of all we observe that the following formulas due to Volevich hold:

$$
a\left(\xi^{\prime}, x_{N}\right) \hat{h}(0)=-\int_{0}^{ \pm \infty}\left\{\left(\partial_{N} a\right)\left(\xi^{\prime}, x_{N}+y_{N}\right) \hat{h}\left(y_{N}\right)+a\left(\xi^{\prime}, x_{N}+y_{N}\right) \widehat{\partial_{N} h}\left(\xi^{\prime}, y_{N}\right)\right\} d y_{N}
$$

where $\partial_{j}=\partial / \partial x_{j}$. Using the identity $1=\frac{\gamma_{0}^{ \pm} \lambda}{\mu_{ \pm} B_{ \pm}^{2}}-\sum_{m=1}^{N-1} \frac{\left(i \xi_{m}\right)\left(i \xi_{m}\right)}{B_{ \pm}^{2}}$, we write

$$
\begin{aligned}
a\left(\xi^{\prime}, x_{N}\right) \hat{h}\left(\xi^{\prime}, 0\right)= & -\int_{0}^{ \pm \infty} a\left(\xi^{\prime}, x_{N}+y_{N}\right) \widehat{\partial_{N} h}\left(\xi^{\prime}, y_{N}\right) d y_{N} \\
& -\int_{0}^{ \pm \infty} \frac{\left(\partial_{N} a\right)\left(\xi^{\prime}, x_{N}+y_{N}\right) \gamma_{0}^{ \pm} \lambda^{1 / 2}}{\mu_{ \pm} B_{ \pm}^{2}} \lambda^{1 / 2} \hat{h}\left(\xi^{\prime}, y_{N}\right) d y_{N} \\
& +\sum_{\ell=1}^{N-1} \int_{0}^{ \pm \infty} \frac{\left(\partial_{N} a\right)\left(\xi^{\prime}, x_{N}+y_{N}\right) i \xi_{\ell}}{B_{ \pm}^{2}} \widehat{\partial_{\ell} h}\left(\xi^{\prime}, y_{N}\right) d y_{N}
\end{aligned}
$$

Let $\mathcal{F}_{\xi^{\prime}}^{-1}$ denote the partial Fourier inverse transform with respect to $\xi^{\prime}$ variable and let $f_{2}$ and $f_{3}=\left(f_{31}, \ldots, f_{3 N}\right)$ be corresponding variables to $\lambda^{1 / 2} h$ and $\nabla h=\left(\partial_{1} h, \ldots, \partial_{N} h\right)$. If we define $A^{ \pm}\left(f_{2}, f_{3}\right)$ by

$$
\begin{aligned}
A^{ \pm}[a]\left(f_{2}, f_{3}\right)= & -\int_{0}^{ \pm \infty} \mathcal{F}_{\xi^{\prime}}^{-1}\left[a\left(\xi^{\prime}, x_{N}+y_{N}\right) \hat{f}_{3 N}\left(\xi^{\prime}, y_{N}\right)\right] d y_{N} \\
& -\int_{0}^{ \pm \infty} \mathcal{F}^{-1}\left[\frac{\left(\partial_{N} a\right)\left(\xi^{\prime}, x_{N}+y_{N}\right) \gamma_{0}^{ \pm} \lambda^{1 / 2}}{\mu_{ \pm} B_{ \pm}^{2}} \hat{f}_{2}\left(\xi^{\prime}, y_{N}\right)\right] d y_{N} \\
& +\sum_{\ell=1}^{N-1} \int_{0}^{ \pm \infty} \mathcal{F}_{\xi^{\prime}}^{-1}\left[\frac{\left(\partial_{N} a\right)\left(\xi^{\prime}, x_{N}+y_{N}\right) i \xi_{\ell}}{B_{ \pm}^{2}} \hat{f}_{3 \ell}\left(\cdot, y_{N}\right)\right] d y_{N}
\end{aligned}
$$

then we have

$$
\mathcal{F}_{\xi^{\prime}}^{-1}\left[a\left(\xi^{\prime}, x_{N}\right) \hat{h}\left(\xi^{\prime}, 0\right)\right]=A^{ \pm}[a]\left(\lambda^{1 / 2} h, \nabla h\right) .
$$

Analogously, using the identity $1=\frac{\gamma_{0}^{ \pm} \lambda}{\mu_{ \pm} B_{ \pm}^{2}}-\sum_{m=1}^{N-1} \frac{\left(i \xi_{m}\right)\left(i \xi_{m}\right)}{B_{ \pm}^{2}}$, we write

$$
\begin{aligned}
a\left(\xi^{\prime}, x_{N}\right) \hat{k}\left(\xi^{\prime}, 0\right) & \\
= & -\int_{0}^{ \pm \infty} a\left(\xi^{\prime}, x_{N}+y_{N}\right)\left[\frac{\gamma_{0}^{ \pm} \lambda^{1 / 2} \widehat{\partial_{N} k}\left(\xi^{\prime}, y_{N}\right)}{\mu_{ \pm} B_{ \pm}^{2}}-\sum_{\ell=1}^{N-1} \frac{i \xi_{\ell} \widehat{\partial_{\ell} \partial_{N} k}\left(\xi^{\prime}, y_{N}\right)}{B_{ \pm}}\right] d y_{N} \\
& -\int_{0}^{ \pm \infty}\left(\partial_{N} a\right)\left(\xi^{\prime}, x_{N}+y_{N}\right)\left[\frac{\gamma_{0}^{ \pm} \lambda \hat{k}\left(\xi^{\prime}, y_{N}\right)}{\mu_{ \pm} B_{ \pm}^{2}}-\sum_{\ell=1}^{N-1} \frac{\widehat{\partial_{\ell} \partial_{\ell} k}\left(\xi^{\prime}, y_{N}\right)}{B_{ \pm}^{2}}\right] d y_{N} .
\end{aligned}
$$


Let $f_{4}, f_{5}=\left(f_{51}, \ldots, f_{5 N}\right)$ and $f_{6}=\left(f_{6 \ell m} \mid \ell, m=1, \ldots, N\right)$ be the corresponding variables to $\lambda k$, $\lambda^{1 / 2} \nabla k$ and $\nabla^{2} k=\left(\partial_{\ell} \partial_{m} k \mid \ell, m=1, \ldots, N\right)$. If we define $B^{ \pm}\left(f_{4}, f_{5}, f_{6}\right)$ by

$$
\begin{aligned}
B^{ \pm}[a]\left(f_{4}, f_{5}, f_{6}\right) & \\
= & -\int_{0}^{ \pm \infty} \mathcal{F}_{\xi^{\prime}}^{-1}\left[a\left(\xi^{\prime}, x_{N}+y_{N}\right)\left\{\frac{\gamma_{0}^{ \pm} \hat{f}_{5 N}\left(\xi^{\prime}, y_{N}\right)}{\mu_{ \pm} B_{ \pm}^{2}}-\sum_{\ell=1}^{N-1} \frac{i \xi_{2} \hat{f}_{6 \ell N}\left(\xi^{\prime}, y_{N}\right)}{B_{ \pm}}\right\}\right] d y_{N} \\
& -\int_{0}^{ \pm \infty} \mathcal{F}_{\xi^{\prime}}^{-1}\left[\left(\partial_{N} a\right)\left(\xi^{\prime}, x_{N}+y_{N}\right)\left\{\frac{\gamma_{0}^{ \pm} \hat{f}_{4}\left(\xi^{\prime}, y_{N}\right)}{\mu_{ \pm} B_{ \pm}^{2}}-\sum_{\ell=1}^{N-1} \frac{\hat{f}_{6 \ell \ell}\left(\xi^{\prime}, y_{N}\right)}{B_{ \pm}^{2}}\right\}\right] d y_{N},
\end{aligned}
$$

then we have

$$
\mathcal{F}_{\xi^{\prime}}^{-1}\left[a\left(\xi^{\prime}, x_{N}\right) \hat{k}\left(\xi^{\prime}, 0\right)\right]=B^{ \pm}[a]\left(\lambda k, \lambda^{1 / 2} \nabla k, \nabla^{2} k\right)
$$

Let us define $u_{j i}^{+}(i=1,2,3,4), u_{J i}^{-}(i=1,2,3)$ and $u_{J 4}^{+}$by $u_{J i}^{ \pm}=\mathcal{F}_{\xi^{\prime}}^{-1}\left[\hat{u}_{J i}^{ \pm}\right](i=1,2,3)$ and $u_{J 4}^{+}=\mathcal{F}_{\xi^{\prime}}^{-1}\left[\hat{u}_{J 4}^{+}\right]$, respectively. Setting $u_{J_{+}}=\sum_{i=1}^{4} u_{J i}^{+}, u_{J-}=\sum_{i=1}^{3} u_{J i}^{-}$and $p_{-}=\mathcal{F}_{\xi^{\prime}}^{-1}\left[\hat{p}_{-}\right]$, by $(2.7)$ we see that $\mathbf{u}_{ \pm}=\left(u_{1 \pm}, \ldots, u_{N \pm}\right)$ and $p_{-}$satisfy (2.1). According to the formulas (2.30), (2.31), (2.32), and (2.33), we define our solution operators $\mathcal{S}_{J i}^{+}(\lambda)(i=1,2,3,4), \mathcal{S}_{J i}^{-}(\lambda)(i=1,2,3)$ and $\mathcal{P}_{-}(\lambda)$ of problem $(2.1)$ such that

$$
\begin{array}{ll}
u_{J i}^{ \pm}=\mathcal{S}_{J i}^{ \pm}(\lambda)\left(\lambda^{1 / 2} \mathbf{h}, \nabla \mathbf{h}, \lambda \mathbf{k}, \lambda^{1 / 2} \nabla \mathbf{k}, \nabla^{2} \mathbf{k}\right) & \text { on } \mathbb{R}_{ \pm}^{N}(i=1,2,3), \\
u_{J 4}^{+}=\mathcal{S}_{J 4}^{+}(\lambda)\left(\lambda^{1 / 2} \mathbf{h}, \nabla \mathbf{h}, \lambda \mathbf{k}, \lambda^{1 / 2} \nabla \mathbf{k}, \nabla^{2} \mathbf{k}\right) & \text { on } \mathbb{R}_{+}^{N} \\
p_{-}=\mathcal{P}_{-}(\lambda)\left(\lambda^{1 / 2} \mathbf{h}, \nabla \mathbf{h}, \lambda \mathbf{k}, \lambda^{1 / 2} \nabla \mathbf{k}, \nabla^{2} \mathbf{k}\right) & \text { on } \mathbb{R}_{-}^{N}
\end{array}
$$

as follows: Note that

$$
\begin{aligned}
& \partial_{N} M_{ \pm}\left(x_{N}+y_{N}\right)=\mp\left(e^{ \pm B_{ \pm}\left(x_{N}+y_{N}\right)}+A_{ \pm} M_{ \pm}\left(x_{N}+y_{N}\right)\right), \\
& \partial_{N} e^{A\left(x_{N}+y_{N}\right)}=A e^{A\left(x_{N}+y_{N}\right)}, \\
& \partial_{N} e^{\mp B_{ \pm}\left(x_{N}+y_{N}\right)}=\mp B_{ \pm} e^{\mp B_{ \pm}\left(x_{N}+y_{N}\right)},
\end{aligned}
$$

where we have set $A_{-}=A$. Let $F_{2}=\left(F_{2 j} \mid j=1, \ldots, N\right), F_{3}=\left(F_{3 \ell m} \mid \ell, m=1, \ldots, N\right), F_{4}=$ $\left(F_{4 \ell} \mid \ell=1, \ldots, N\right), F_{5}=\left(F_{5 \ell m} \mid \ell, m=1, \ldots, N\right)$ and $F_{6}=\left(F_{6 \ell m n} \mid \ell, m, n=1, \ldots, N\right)$ be the corresponding variables to $\lambda^{1 / 2} \mathbf{h}=\left(\lambda^{1 / 2} h_{1}, \ldots, \lambda^{1 / 2} h_{N}\right), \nabla \mathbf{h}=\left(\partial_{\ell} h_{m} \mid \ell, m=1, \ldots, N\right), \lambda \mathbf{k}=$ $\left(\lambda k_{1}, \ldots, \lambda k_{N}\right), \lambda^{1 / 2} \nabla \mathbf{k}=\left(\lambda^{1 / 2} \partial_{\ell} k_{m} \mid \ell, m=1, \ldots, N\right)$ and $\nabla^{2} \mathbf{k}=\left(\partial_{\ell} \partial_{m} k_{n} \mid \ell, m, n=1, \ldots, N\right)$, respectively. Then we define the operators $\mathcal{S}_{J 1}^{ \pm}(\lambda), \mathcal{S}_{J 2}^{ \pm}(\lambda), \mathcal{S}_{J 3}^{ \pm}(\lambda), \mathcal{S}_{N 4}^{+}(\lambda)$ and $\mathcal{P}_{-}(\lambda)$ by

$$
\begin{aligned}
\mathcal{S}_{J 1}^{ \pm}(\lambda)\left(F_{2}, F_{3}, F_{4}, F_{5}, F_{6}\right) \\
=-\sum_{\ell=1}^{N}\left\{\int _ { 0 } ^ { \pm \infty } \mathcal { F } _ { \xi ^ { \prime } } ^ { - 1 } \left[A M _ { \pm } ( x _ { N } + y _ { N } ) \left[R_{J \ell,-1}^{ \pm} \hat{F}_{3 N \ell}\left(\xi^{\prime}, y_{N}\right)+\frac{R_{J \ell, 0}^{ \pm} \gamma_{0}^{ \pm} \lambda^{1 / 2}}{\mu_{ \pm} B_{ \pm}^{2}} \hat{F}_{5 N \ell}\left(\xi^{\prime}, y_{N}\right)\right.\right.\right. \\
\left.\left.\quad-\sum_{m=1}^{N-1} \frac{R_{J \ell, 0}^{ \pm}\left(i \xi_{m}\right)}{B_{ \pm}^{2}} \hat{F}_{6 m N \ell}\left(\xi^{\prime}, y_{N}\right)\right]\right] d y_{N} \\
\quad \pm \int_{0}^{ \pm \infty} \mathcal{F}_{\xi^{\prime}}^{-1}\left[( A e ^ { \mp B _ { \pm } ( x _ { N } + y _ { N } ) } + A A _ { \pm } M _ { \pm } ( x _ { N } + y _ { N } ) ) \left[\frac{R_{J \ell,-1}^{ \pm} \gamma_{0}^{ \pm} \lambda^{1 / 2}}{\mu_{ \pm} B_{ \pm}^{2}} \hat{F}_{2 \ell}\left(\xi^{\prime}, y_{N}\right)\right.\right.
\end{aligned}
$$




$$
\begin{aligned}
& -\sum_{m=1}^{N-1} \frac{R_{J \ell,-1}^{ \pm}\left(i \xi_{m}\right)}{B_{ \pm}^{2}} \hat{F}_{3 m \ell}\left(\xi^{\prime}, y_{N}\right)+\frac{R_{J \ell, 0}^{ \pm} \gamma_{0}^{ \pm}}{\mu_{ \pm} B_{ \pm}^{2}} \hat{F}_{4 \ell}\left(\xi^{\prime}, y_{N}\right) \\
& \left.\left.\left.-\sum_{m=1}^{N-1} \frac{R_{J \ell, 0}^{ \pm}}{B_{ \pm}^{2}} \hat{F}_{6 m m \ell}\left(\xi^{\prime}, y_{N}\right)\right]\right] d y_{N}\right\} \\
& \mathcal{S}_{J 2}^{ \pm}(\lambda)\left(F_{2}, F_{3}, F_{4}, F_{5}, F_{6}\right) \\
& =-\sum_{\ell=1}^{N}\left\{\int _ { 0 } ^ { \pm \infty } \mathcal { F } _ { \xi ^ { \prime } } ^ { - 1 } \left[A e ^ { \mp B _ { \pm } ( x _ { N } + y _ { N } ) } \left[S_{J \ell,-2}^{ \pm} \hat{F}_{3 N \ell}\left(\xi^{\prime}, y_{N}\right)+\frac{S_{J \ell,-1}^{ \pm} \gamma_{0}^{ \pm} \lambda^{1 / 2}}{\mu_{ \pm} B_{ \pm}^{2}} \hat{F}_{5 N \ell}\left(\xi^{\prime}, y_{N}\right)\right.\right.\right. \\
& \left.\left.-\sum_{m=1}^{N-1} \frac{S_{J \ell,-1}^{ \pm}\left(i \xi_{m}\right)}{B_{ \pm}^{2}} \hat{F}_{6 m N \ell}\left(\xi^{\prime}, y_{N}\right)\right]\right] d y_{N} \\
& \pm \int_{0}^{ \pm \infty} \mathcal{F}_{\xi^{\prime}}^{-1}\left[B _ { \pm } A e ^ { \mp B _ { \pm } ( x _ { N } + y _ { N } ) } \left[\frac{S_{J \ell,-2}^{ \pm} \gamma_{0}^{ \pm} \lambda^{1 / 2}}{\mu_{ \pm} B_{ \pm}^{2}} \hat{F}_{2 \ell}\left(\xi^{\prime}, y_{N}\right)\right.\right. \\
& -\sum_{m=1}^{N-1} \frac{S_{J \ell,-2}^{ \pm}\left(i \xi_{m}\right)}{B_{ \pm}^{2}} \hat{F}_{3 m \ell}\left(\xi^{\prime}, y_{N}\right)+\frac{S_{\ell \ell,-1}^{ \pm} \gamma_{0}^{ \pm}}{\mu_{ \pm} B_{ \pm}^{2}} \hat{F}_{4 \ell}\left(\xi^{\prime}, y_{N}\right) \\
& \left.\left.\left.-\sum_{m=1}^{N-1} \frac{S_{J \ell,-1}^{ \pm}}{B_{ \pm}^{2}} \hat{F}_{6 m m \ell}\left(\xi^{\prime}, y_{N}\right)\right]\right] d y_{N}\right\} \\
& \mathcal{S}_{J 3}^{ \pm}(\lambda)\left(F_{2}, F_{3}, F_{4}, F_{5}, F_{6}\right) \\
& =-\left\{\int _ { 0 } ^ { \pm \infty } \mathcal { F } _ { \xi ^ { \prime } } ^ { - 1 } \left[e ^ { \mp B _ { \pm } ( x _ { N } + y _ { N } ) } \left[T_{J,-1}^{ \pm} \hat{F}_{3 N J}\left(\xi^{\prime}, y_{N}\right)+\frac{T_{J, 0}^{ \pm} \gamma_{0}^{ \pm} \lambda^{1 / 2}}{\mu_{ \pm} B_{ \pm}^{2}} \hat{F}_{5 N J}\left(\xi^{\prime}, y_{N}\right)\right.\right.\right. \\
& \left.\left.-\sum_{m=1}^{N-1} \frac{T_{J, 0}^{ \pm}\left(i \xi_{m}\right)}{B_{ \pm}^{2}} \hat{F}_{6 m N J}\left(\xi^{\prime}, y_{N}\right)\right]\right] d y_{N} \\
& \pm \int_{0}^{ \pm \infty} \mathcal{F}_{\xi^{\prime}}^{-1}\left[B _ { \pm } e ^ { \mp B _ { \pm } ( x _ { N } + y _ { N } ) } \left[\frac{T_{J,-1}^{ \pm} \gamma_{0}^{ \pm} \lambda^{1 / 2}}{\mu_{ \pm} B_{ \pm}^{2}} \hat{F}_{2 J}\left(\xi^{\prime}, y_{N}\right)\right.\right. \\
& -\sum_{m=1}^{N-1} \frac{T_{J,-1}^{ \pm}\left(i \xi_{m}\right)}{B_{ \pm}^{2}} \hat{F}_{3 m J}\left(\xi^{\prime}, y_{N}\right)+\frac{T_{J, 0}^{ \pm} \gamma_{0}^{ \pm}}{\mu_{ \pm} B_{ \pm}^{2}} \hat{F}_{4 J}\left(\xi^{\prime}, y_{N}\right) \\
& \left.\left.\left.-\sum_{m=1}^{N-1} \frac{T_{J, 0}^{ \pm}}{B_{ \pm}^{2}} \hat{F}_{6 m m J}\left(\xi^{\prime}, y_{N}\right)\right]\right] d y_{N}\right\}, \\
& \mathcal{S}_{j 4}^{+}(\lambda)\left(F_{2}, F_{3}, F_{4}, F_{5}, F_{6}\right)=0 \text {, } \\
& \mathcal{S}_{N 4}^{+}(\lambda)\left(F_{2}, F_{3}, F_{4}, F_{5}, F_{6}\right) \\
& =-\left\{\int _ { 0 } ^ { \infty } \mathcal { F } _ { \xi ^ { \prime } } ^ { - 1 } \left[A _ { + } M _ { + } ( x _ { N } + y _ { N } ) \left[\frac{U_{N, 0}^{+} \gamma_{0}^{+} \lambda^{1 / 2}}{\mu_{+} B_{+}^{2}} \hat{F}_{5 N J}\left(\xi^{\prime}, y_{N}\right)\right.\right.\right. \\
& \left.\left.-\sum_{m=1}^{N-1} \frac{U_{N, 0}^{+}\left(i \xi_{m}\right)}{B_{+}^{2}} \hat{F}_{6 m N J}\left(\xi^{\prime}, y_{N}\right)\right]\right] d y_{N} \\
& -\int_{0}^{ \pm \infty} \mathcal{F}_{\xi^{\prime}}^{-1}\left[A_{+}\left(e^{-B_{+}\left(x_{N}+y_{N}\right)}+A_{+} M_{+}\left(x_{N}+y_{N}\right)\right)\right. \\
& \left.\left.\times\left[\frac{U_{N, 0}^{+} \gamma_{0}^{+}}{\mu_{+} B_{+}^{2}} \hat{F}_{4 J}\left(\xi^{\prime}, y_{N}\right)-\sum_{m=1}^{N-1} \frac{U_{N, 0}^{+}}{B_{+}^{2}} \hat{F}_{6 m m J}\left(\xi^{\prime}, y_{N}\right)\right]\right] d y_{N}\right\},
\end{aligned}
$$




$$
\begin{aligned}
\mathcal{P}_{-}(\lambda)\left(F_{2}, F_{3}, F_{4}, F_{5}, F_{6}\right) & \sum_{\ell=1}^{N}\left\{\int _ { - \infty } ^ { 0 } \mathcal { F } _ { \xi ^ { \prime } } ^ { - 1 } \left[e ^ { A ( x _ { N } + y _ { N } ) } \left[p_{\ell, 0}^{-} \hat{F}_{3 N \ell}\left(\xi^{\prime}, y_{N}\right)+\frac{p_{\ell, 1}^{-} \gamma_{0}^{-} \lambda^{1 / 2}}{\mu_{-} B_{-}^{2}} \hat{F}_{5 N \ell}\left(\xi^{\prime}, y_{N}\right)\right.\right.\right. \\
& \left.\left.-\sum_{m=1}^{N-1} \frac{p_{\ell, 1}^{-}\left(i \xi_{m}\right)}{B_{-}^{2}} \hat{F}_{6 m N \ell}\left(\xi^{\prime}, y_{N}\right)\right]\right] d y_{N} \\
& +\int_{-\infty}^{0} \mathcal{F}_{\xi^{\prime}}^{-1}\left[A e ^ { A ( x _ { N } + y _ { N } ) } \left[\frac{p_{\ell, 0}^{-} \gamma_{0}^{-} \lambda^{1 / 2}}{\mu-B_{-}^{2}} \hat{F}_{2 \ell}\left(\xi^{\prime}, y_{N}\right)-\sum_{m=1}^{N-1} \frac{p_{\zeta \ell, 0}^{-}\left(i \xi_{m}\right)}{B_{-}^{2}} \hat{F}_{3 m \ell}\left(\xi^{\prime}, y_{N}\right)\right.\right. \\
& \left.\left.\left.+\frac{p_{\ell, 1}^{-} \gamma_{0}^{-}}{\mu_{-} B_{-}^{2}} \hat{F}_{4 \ell}\left(\xi^{\prime}, y_{N}\right)-\sum_{m=1}^{N-1} \frac{p_{\ell, 1}^{-}}{B_{-}^{2}} \hat{F}_{6 m m \ell}\left(\xi^{\prime}, y_{N}\right)\right]\right] d y_{N}\right\}
\end{aligned}
$$

Obviously, by (2.31) and (2.33), we have (2.34).

If we define operators $\mathcal{S}_{ \pm}(\lambda)$ by

$$
\mathcal{S}_{+}(\lambda) \mathbf{F}^{\prime}=\sum_{i=1}^{4}\left(\mathcal{S}_{1 i}^{+}(\lambda) \mathbf{F}^{\prime}, \ldots, \mathcal{S}_{N i}^{+}(\lambda) \mathbf{F}^{\prime}\right), \quad \mathcal{S}_{-}(\lambda) \mathbf{F}^{\prime}=\sum_{i=1}^{3}\left(\mathcal{S}_{1 i}^{-}(\lambda) \mathbf{F}^{\prime}, \ldots, \mathcal{S}_{N i}^{+}(\lambda) \mathbf{F}^{\prime}\right)
$$

with $\mathbf{F}^{\prime}=\left(F_{2}, F_{3}, F_{4}, F_{5}, F_{6}\right)$, respectively, by $(2.34)$ we have

$$
\mathbf{u}_{ \pm}=\mathcal{S}_{ \pm}(\lambda)\left(\lambda^{1 / 2} \mathbf{h}, \nabla \mathbf{h}, \lambda \mathbf{k}, \lambda^{1 / 2} \nabla \mathbf{k}, \nabla^{2} \mathbf{k}\right)
$$

Moreover, if we set

$$
\mathcal{Z}_{q}\left(\mathbb{R}^{N}\right)=\left\{\left(F_{2}, F_{3}, F_{4}, F_{5}, F_{6}\right) \mid F_{2}, F_{4} \in L_{q}\left(\mathbb{R}^{N}\right), F_{3}, F_{5} \in L_{q}\left(\mathbb{R}^{N}\right)^{N^{2}}, F_{6} \in L_{q}\left(\mathbb{R}^{N}\right)^{N^{3}}\right\},
$$

then, using Lemma 3.1 and Lemma 3.2 in Section 3, we have

$$
\begin{aligned}
& \mathcal{R}_{\mathcal{L}\left(\mathcal{Z}\left(\mathbb{R}^{N}\right), L_{q}\left(\mathbb{R}_{ \pm}^{N}\right)^{2 N+N^{2}+N^{3}}\right)}\left(\left\{\left(\tau \partial_{\tau}\right)^{\ell} G_{\lambda} \mathcal{S}_{ \pm J}(\lambda) \mid \lambda \in \Lambda_{\epsilon, \lambda_{0}}\right\}\right) \leq C \quad(\ell=0,1), \\
& \mathcal{R}_{\mathcal{L}\left(\mathcal{Z}\left(\mathbb{R}^{N}\right), L_{q}\left(\mathbb{R}_{ \pm}^{N}\right)^{N}\right)}\left(\left\{\left(\tau \partial_{\tau}\right)^{\ell} \nabla \mathcal{P}_{-}(\lambda) \mid \lambda \in \Lambda_{\epsilon, \lambda_{0}}\right\}\right) \leq C \quad(\ell=0,1) .
\end{aligned}
$$

The estimates (2.38) are proved in Section 6 below.

\section{Technical lemmas}

To prove the $\mathcal{R}$-boundedness of solution operators, we use the following two lemmas. The first lemma is used to show the $\mathcal{R}$-boundedness of the compressible part and the second one to show that of the incompressible part.

Lemma 3.1 Let $n_{1}$ and $n_{2}$ be multipliers belonging to $\mathbf{M}_{-2,2}$ and $\mathbf{M}_{-1,1}$, respectively. Let $K_{i}^{+}$ $(i=1,2,3,4)$ be operators defined by

$$
\begin{aligned}
& K_{1}^{+}(\lambda) g=\int_{0}^{\infty} \mathcal{F}_{\xi^{\prime}}^{-1}\left[n_{1}\left(\lambda, \xi^{\prime}\right) A A_{+} M_{+}\left(x_{N}+y_{N}\right) \hat{g}\left(\xi^{\prime}, y_{N}\right)\right]\left(x^{\prime}\right) d y_{N}, \\
& K_{2}^{+}(\lambda) g=\int_{0}^{\infty} \mathcal{F}_{\xi^{\prime}}^{-1}\left[n_{1}\left(\lambda, \xi^{\prime}\right) A e^{-B_{+}\left(x_{N}+y_{N}\right)} \hat{g}\left(\xi^{\prime}, y_{N}\right)\right]\left(x^{\prime}\right) d y_{N},
\end{aligned}
$$




$$
\begin{aligned}
& K_{3}^{+}(\lambda) g=\int_{0}^{\infty} \mathcal{F}_{\xi^{\prime}}^{-1}\left[n_{2}\left(\lambda, \xi^{\prime}\right) A_{+} M_{+}\left(x_{N}+y_{N}\right) \hat{g}\left(\xi^{\prime}, y_{N}\right)\right]\left(x^{\prime}\right) d y_{N}, \\
& K_{4}^{+}(\lambda) g=\int_{0}^{\infty} \mathcal{F}_{\xi^{\prime}}^{-1}\left[n_{2}\left(\lambda, \xi^{\prime}\right) e^{-B_{+}\left(x_{N}+y_{N}\right)} \hat{g}\left(\xi^{\prime}, y_{N}\right)\right]\left(x^{\prime}\right) d y_{N} .
\end{aligned}
$$

Then there exists a constant $C$ such that

$$
\mathcal{R}_{\mathcal{L}\left(L_{q}\left(\mathbb{R}_{+}^{N}\right), L_{q}\left(\mathbb{R}_{+}^{N}\right)^{\left.1+N+N^{2}\right)}\right)}\left(\left\{\left(\tau \partial_{\tau}\right)^{\ell} G_{\lambda} K_{i}^{+}(\lambda) \mid \lambda \in \Lambda\right\}\right) \leq C \quad(\ell=0,1, i=1,2,3,4) .
$$

Lemma 3.2 Let $n_{3}, n_{4}$, and $n_{5}$ be multipliers belonging to $\mathbf{M}_{-1,2}, \mathbf{M}_{-2,2}$ and $\mathbf{M}_{-1,1}$, respectively. Let $K_{i}^{+}(i=1,2,3,4)$ be operators defined by

$$
\begin{aligned}
& K_{1}^{-}(\lambda) g=\int_{-\infty}^{0} \mathcal{F}_{\xi^{\prime}}^{-1}\left[n_{3}\left(\lambda, \xi^{\prime}\right) A M_{-}\left(x_{N}+y_{N}\right) \hat{g}\left(\xi^{\prime}, y_{N}\right)\right]\left(x^{\prime}\right) d y_{N}, \\
& K_{2}^{-}(\lambda) g=\int_{-\infty}^{0} \mathcal{F}_{\xi^{\prime}}^{-1}\left[n_{4}\left(\lambda, \xi^{\prime}\right) A e^{B_{-}\left(x_{N}+y_{N}\right)} \hat{g}\left(\xi^{\prime}, y_{N}\right)\right]\left(x^{\prime}\right) d y_{N}, \\
& K_{3}^{-}(\lambda) g=\int_{-\infty}^{0} \mathcal{F}_{\xi^{\prime}}^{-1}\left[n_{4}\left(\lambda, \xi^{\prime}\right) A^{2} M_{-}\left(x_{N}+y_{N}\right) \hat{g}\left(\xi^{\prime}, y_{N}\right)\right]\left(x^{\prime}\right) d y_{N}, \\
& K_{4}^{-}(\lambda) g=\int_{-\infty}^{0} \mathcal{F}_{\xi^{\prime}}^{-1}\left[n_{5}\left(\lambda, \xi^{\prime}\right) e^{B_{-}\left(x_{N}+y_{N}\right)} \hat{g}\left(\xi^{\prime}, y_{N}\right)\right]\left(x^{\prime}\right) d y_{N} .
\end{aligned}
$$

Then there exists a constant $C$ such that

$$
\begin{aligned}
& \mathcal{R}_{\mathcal{L}\left(L_{q}\left(\mathbb{R}_{-}^{N}\right), L_{q}\left(\mathbb{R}_{-}^{N}\right)^{1+N+N^{2}}\right)}\left(\left\{\left(\tau \partial_{\tau}\right)^{\ell} G_{\lambda} K_{i}^{-}(\lambda) \mid \lambda \in \Lambda\right\}\right) \\
& \quad \leq C \quad(\ell=0,1, i=1,2,3,4) .
\end{aligned}
$$

The assertions for $K_{1}^{+}$and $K_{2}^{+}$in Lemma 3.1 immediately follows from the following lemma.

Lemma 3.3 Let $m(\lambda)$ be a multiplier belonging to $\mathbf{M}_{0,2}$. Let $L_{1}(\lambda)$ and $L_{2}(\lambda)$ be operators defined by

$$
\begin{aligned}
& L_{1}(\lambda) g=\int_{0}^{\infty} \mathcal{F}_{\xi^{\prime}}^{-1}\left[m\left(\lambda, \xi^{\prime}\right) A A_{+} M_{+}\left(x_{N}+y_{N}\right) \hat{g}\left(\xi^{\prime}, y_{N}\right)\right]\left(x^{\prime}\right) d y_{N}, \\
& L_{2}(\lambda) g=\int_{0}^{\infty} \mathcal{F}_{\xi^{\prime}}^{-1}\left[m\left(\lambda, \xi^{\prime}\right) A e^{-B_{+}\left(x_{N}+y_{N}\right)} \hat{g}\left(\xi^{\prime}, y_{N}\right)\right]\left(x^{\prime}\right) d y_{N} .
\end{aligned}
$$

Then we have

$$
\mathcal{R}_{\mathcal{L}\left(L_{q}\left(\mathbb{R}_{+}^{N}\right)\right)}\left(\left\{\left(\tau \partial_{\tau}\right)^{\ell} L_{i}(\lambda) \mid \lambda \in \Lambda\right\}\right) \leq C \quad(\ell=0,1, i=1,2) .
$$

Proof Set $\psi_{i}(\lambda, x)=\mathcal{F}_{\xi^{\prime}}^{-1}\left[m\left(\lambda, \xi^{\prime}\right) A N_{i}\left(\lambda, \xi^{\prime}, x_{N}\right)\right]\left(x^{\prime}\right)$ with $N_{1}=A_{+} M_{+}\left(x_{N}\right)$ and $N_{2}=e^{-B_{+} x_{N}}$. As was seen in Shibata and Shimizu [19, Proof of Lemma 5.4], the lemma follows from the fact that

$$
\left|\left(\tau \partial_{\tau}\right)^{\ell} \psi_{i}(\lambda, x)\right| \leq C|x|^{-N} \quad(\ell=0,1)
$$


Thus, we prove (3.2). Using the following Bell formula for the derivatives of the composite function of $f(t)$ and $t=g\left(\xi^{\prime}\right)$ :

$$
\partial_{\xi^{\prime}}^{\kappa^{\prime}} f\left(g\left(\xi^{\prime}\right)\right)=\sum_{\ell=1}^{\left|\kappa^{\prime}\right|} f^{(\ell)}\left(g\left(\xi^{\prime}\right)\right) \sum_{\substack{\kappa_{1}^{\prime}+\cdots+\kappa_{\ell}^{\prime}=\kappa^{\prime} \\ \mid \kappa_{i}^{\prime} \geq 1}} \Gamma_{\kappa_{1}^{\prime}, \ldots, \kappa_{\ell}^{\prime}}^{\kappa^{\prime}}\left(\partial_{\xi^{\prime}}^{\kappa_{1}^{\prime}} g\left(\xi^{\prime}\right)\right) \cdots\left(\partial_{\xi^{\prime}}^{\kappa_{\ell}^{\prime}} g\left(\xi^{\prime}\right)\right)
$$

with $f^{(\ell)}(t)=d^{\ell} f(t) / d t^{\ell}$ and suitable coefficients $\Gamma_{\kappa_{1}^{\prime}, \ldots, \kappa_{\ell}^{\prime}}^{\kappa^{\prime}}$, we have

$$
\left|\partial_{\xi^{\prime}}^{\alpha^{\prime}}\left[\int_{0}^{1} e^{-\left((1-\theta) A_{+}+\theta B_{+}\right) x_{N}} d \theta\right]\right| \leq C_{\alpha^{\prime}}\left(|\lambda|^{1 / 2}+A\right)^{-\left|\kappa^{\prime}\right|} e^{-c\left(|\lambda|^{1 / 2}+A\right) x_{N}}
$$

with some positive constant $c$ independent of $\kappa^{\prime}$. Thus, we have

$$
\left|\partial_{\xi^{\prime}}^{\kappa^{\prime}} N_{i}\right| \leq C_{\alpha^{\prime}}\left(|\lambda|^{1 / 2}+A\right)^{-\left|\kappa^{\prime}\right|} e^{-(c / 2)\left(|\lambda|^{1 / 2}+A\right) x_{N}} .
$$

To prove the estimate

$$
\left|\psi_{i}(\lambda, x)\right| \leq C\left|x^{\prime}\right|^{-N}
$$

using the identity $e^{i x^{\prime} \cdot \xi^{\prime}}=\sum_{\ell=1}^{N-1} \frac{x_{\ell}}{i\left|x^{\prime}\right|^{2}}\left(i x_{\ell}\right)$, we write

$$
\psi_{i}(\lambda, x)=\sum_{\ell=1}^{N-1} \frac{i x_{\ell}}{\left|x^{\prime}\right|^{2}} \frac{1}{(2 \pi)^{N-1}} \int_{\mathbb{R}^{N-1}} e^{i x^{\prime} \cdot \xi^{\prime}} \frac{\partial}{\partial \xi_{\ell}}\left(m\left(\lambda, \xi^{\prime}\right) A N_{i}\left(\lambda, \xi^{\prime}, x_{N}\right)\right) d \xi^{\prime}
$$

Since $m \in \mathbf{M}_{0,2}$, by (2.6) and (3.4) we have

$$
\left|\partial_{\xi^{\prime}}^{\kappa^{\prime}} \frac{\partial}{\partial \xi_{\ell}}\left(m\left(\lambda, \xi^{\prime}\right) A N_{i}\left(\lambda, \xi^{\prime}, x_{N}\right)\right)\right| \leq C_{\kappa^{\prime}} A^{-\left|\kappa^{\prime}\right|} e^{-(c / 2)\left(\left.|\lambda|\right|^{1 / 2}+A\right) x_{N}} .
$$

Thus, by Theorem 2.2 due to Shibata and Shimizu [20], we have

$$
\left|\int_{\mathbb{R}^{N-1}} e^{i x^{\prime} \cdot \xi^{\prime}} \frac{\partial}{\partial \xi_{\ell}}\left(m\left(\lambda, \xi^{\prime}\right) A N_{i}\left(\lambda, \xi^{\prime}, x_{N}\right)\right) d \xi^{\prime}\right| \leq C\left|x^{\prime}\right|^{-(N-1)}
$$

from which we have (3.5).

On the other hand, by (2.6) with $\kappa^{\prime}=0$ and (3.4) with $\kappa^{\prime}=0$, we have

$$
\left|\psi_{i}(\lambda, x)\right| \leq C \int_{\mathbb{R}^{N-1}}\left|\xi^{\prime}\right| e^{-(c / 2)\left|\xi^{\prime}\right| x_{N}} d \xi^{\prime}
$$

Thus, using the change of variables $x_{N} \xi^{\prime}=\eta^{\prime}$, we have $\left|\psi_{i}(\lambda, x)\right| \leq C\left(x_{N}\right)^{-N}$, which, combined with (3.5), furnishes (3.2). Analogously, we have $\left|\tau \partial_{\tau} \psi_{i}(\lambda, x)\right| \leq C|x|^{-N}$, which completes the proof of Lemma 3.3.

The assertions for $K_{3}^{+}$and $K_{4}^{+}$in Lemma 3.1 immediately follow from the following lemma. 
Lemma 3.4 Let $m(\lambda)$ be a multiplier belonging to $\mathbf{M}_{1,2}$. Let $L_{3}(\lambda)$ and $L_{4}(\lambda)$ be operators defined by

$$
\begin{aligned}
& L_{3}(\lambda) g=\int_{0}^{\infty} \mathcal{F}_{\xi^{\prime}}^{-1}\left[m\left(\lambda, \xi^{\prime}\right) A_{+} M_{+}\left(x_{N}+y_{N}\right) \hat{g}\left(\xi^{\prime}, y_{N}\right)\right]\left(x^{\prime}\right) d y_{N}, \\
& L_{4}(\lambda) g=\int_{0}^{\infty} \mathcal{F}_{\xi^{\prime}}^{-1}\left[m\left(\lambda, \xi^{\prime}\right) e^{-B_{+}\left(x_{N}+y_{N}\right)} \hat{g}\left(\xi^{\prime}, y_{N}\right)\right]\left(x^{\prime}\right) d y_{N} .
\end{aligned}
$$

Then we have

$$
\mathcal{R}_{\mathcal{L}\left(L_{q}\left(\mathbb{R}_{+}^{N}\right)\right)}\left(\left\{\left(\tau \partial_{\tau}\right)^{\ell} L_{i}(\lambda) \mid \lambda \in \Lambda\right\}\right) \leq C \quad(\ell=0,1, i=3,4) .
$$

Proof Set $\varphi_{i}(\lambda, x)=\mathcal{F}_{\xi^{\prime}}^{-1}\left[m\left(\lambda, \xi^{\prime}\right) N_{i}\left(\lambda, \xi^{\prime}, x_{N}\right)\right]\left(x^{\prime}\right)$ with $N_{1}=A_{+} M_{+}\left(x_{N}\right)$ and $N_{2}=e^{-B_{+} x_{N}}$. As was stated in the proof of Lemma 3.3, the lemma follows from the fact that

$$
\left|\left(\tau \partial_{\tau}\right)^{\ell} \varphi_{i}(\lambda, x)\right| \leq C|x|^{-N} \quad(\ell=0,1) .
$$

First, we prove that

$$
\left|\varphi_{i}(\lambda, x)\right| \leq C_{N}\left|x^{\prime}\right|^{-N}
$$

By (2.5), (3.4), and the Leibniz rule, we have

$$
\begin{aligned}
\left|\partial_{\xi^{\prime}}^{\kappa^{\prime}} \frac{\partial}{\partial \xi_{\ell}}\left(m\left(\lambda, \xi^{\prime}\right) N_{i}\left(\lambda, \xi^{\prime}, x_{N}\right)\right)\right| & \leq C_{\kappa^{\prime}}\left(|\lambda|^{1 / 2}+A\right)^{-\left|\kappa^{\prime}\right|} e^{-\left(|\lambda|^{1 / 2}+A\right) x_{N}} \\
& \leq C_{\kappa^{\prime}} A^{-\left|\kappa^{\prime}\right|} e^{-\left(|\lambda|^{1 / 2}+A\right) x_{N}},
\end{aligned}
$$

so that by Theorem 2.2 in Shibata and Shimizu [20] we have

$$
\left|\int_{\mathbb{R}^{N-1}} e^{i x^{\prime} \cdot \xi^{\prime}} \frac{\partial}{\partial \xi_{\ell}}\left(m\left(\lambda, \xi^{\prime}\right) N_{i}\left(\lambda, \xi^{\prime}, x_{N}\right)\right) d \xi^{\prime}\right| \leq C\left|x^{\prime}\right|^{-(N-1)} .
$$

Thus, employing the same argumentation as in the proof of Lemma 3.3, we have (3.7).

On the other hand, we have

$$
\begin{aligned}
\left|\varphi_{i}(\lambda, x)\right| & \leq C \int_{\mathbb{R}^{N-1}}\left(|\lambda|^{1 / 2}+\left|\xi^{\prime}\right|\right) e^{-(c / 2)\left(|\lambda|^{1 / 2}+\left|\xi^{\prime}\right|\right) x_{N}} d \xi^{\prime} \\
& \leq C\left(4 /\left(c x_{N}\right)\right) \int_{\mathbb{R}^{N-1}} e^{-(c / 4)\left(|\lambda|^{1 / 2}+\left|\xi^{\prime}\right|\right) x_{N}} d \xi^{\prime} \\
& \leq C\left(4 /\left(c x_{N}\right)\right)^{-N} \int_{\mathbb{R}^{N-1}} e^{-(c / 4)|\lambda|^{1 / 2} x_{N}} e^{-\left|\eta^{\prime}\right|} d \eta^{\prime} .
\end{aligned}
$$

Thus, we have (3.6) with $\ell=0$. Analogously, we have (3.6) with $\ell=1$, which completes the proof of Lemma 3.4.

The assertions for $K_{4}^{-}(\lambda)$ in Lemma 3.2 follows from the same observation as in the proof of Lemma 3.1 for $K_{4}^{+}(\lambda)$. The assertion for $K_{1}^{-}(\lambda), K_{2}^{-}(\lambda)$ and $K_{3}^{-}(\lambda)$ in Lemma 3.2 follows from the following lemma due to Shibata and Shimizu [19, Lemma 5.4]. 
Lemma 3.5 Let $m(\lambda)$ be a multiplier belonging to $\mathbf{M}_{0,2}$. Let $L_{i}(\lambda)(i=5,6,7)$ be operators defined by

$$
\begin{aligned}
& L_{5}(\lambda) g=\int_{-\infty}^{0} \mathcal{F}_{\xi^{\prime}}^{-1}\left[m\left(\lambda, \xi^{\prime}\right) A^{2} M_{-}\left(x_{N}+y_{N}\right) \hat{g}\left(\xi^{\prime}, y_{N}\right)\right]\left(x^{\prime}\right) d y_{N}, \\
& L_{6}(\lambda) g=\int_{-\infty}^{0} \mathcal{F}_{\xi^{\prime}}^{-1}\left[m\left(\lambda, \xi^{\prime}\right) A e^{A\left(x_{N}+y_{N}\right)} \hat{g}\left(\xi^{\prime}, y_{N}\right)\right]\left(x^{\prime}\right) d y_{N}, \\
& L_{7}(\lambda) g=\int_{-\infty}^{0} \mathcal{F}_{\xi^{\prime}}^{-1}\left[m\left(\lambda, \xi^{\prime}\right) A e^{B_{-}\left(x_{N}+y_{N}\right)} \hat{g}\left(\xi^{\prime}, y_{N}\right)\right]\left(x^{\prime}\right) d y_{N} .
\end{aligned}
$$

Then we have

$$
\mathcal{R}_{\mathcal{L}\left(L_{q}\left(\mathbb{R}_{-}^{N}\right)\right)}\left(\left\{\left(\tau \partial_{\tau}\right)^{\ell} L_{i}(\lambda) \mid \lambda \in \Lambda\right\}\right) \leq C \quad(\ell=0,1, i=5,6,7) .
$$

\section{Some estimates of several multipliers}

In this section, we estimate several multipliers. For this purpose, we start with the following lemma.

Lemma 4.1 Let $0<\epsilon<\pi / 2, \lambda_{0}>0, \delta_{0}>0$ and $s \geq 0$.

(1) For any $\lambda \in \Sigma_{\epsilon}, \xi \in \mathbb{R}^{N}$ and $\alpha, \beta>0$, we have $|\alpha \lambda+\beta| \geq\left(\sin \frac{\epsilon}{2}\right)(\alpha|\lambda|+\beta)$.

(2) There exists a number $\sigma \in(0, \pi)$ depending on $s, \mu_{+}, \nu_{+}, \gamma_{1+}, \gamma_{2+}, \lambda_{0}, \delta_{0}$ and $\epsilon$ such that

$$
\left(s \mu_{+}+v_{+}+\delta\right)^{-1} \lambda \in \Sigma_{\sigma} \quad \text { for any } \lambda \in \Gamma_{\epsilon, \lambda} .
$$

(3) There exist constants $\delta_{1}$ and $\delta_{2}$ depending on $s, \mu_{+}, \nu_{+}, \gamma_{1^{+}}, \gamma_{2+}, \lambda_{0}, \delta_{0}$ and $\epsilon$ such that

$$
\begin{aligned}
& \qquad \delta_{1}\left(|\lambda|+\left|\xi^{\prime}\right|^{2}\right) \leq\left.\left|\left(s \mu_{+}+v_{+}+\delta\right)^{-1} \lambda+\right| \xi^{\prime}\right|^{2} \mid \leq \delta_{2}\left(|\lambda|+\left|\xi^{\prime}\right|^{2}\right) \\
& \text { for any }\left(\lambda, \xi^{\prime}\right) \in \tilde{\Gamma}_{\epsilon, \lambda_{0}}=\Gamma_{\epsilon, \lambda_{0}} \times\left(\mathbb{R}^{N-1} \backslash\{0\}\right) .
\end{aligned}
$$

Remark 4.2 Lemma 4.1 was proved in Götz and Shibata [21, Lemma 3.1], so that we may omit its proof.

First we estimate $A_{+}^{s}, B_{ \pm}^{s},\left(A_{+}+B_{+}\right)^{s}$ and $\left(\mu_{+} B_{+}+\mu_{-} B_{-}\right)^{s}$. For this purpose, we use the estimates

$$
c\left(|\lambda|^{1 / 2}+A\right) \leq \operatorname{Re} M_{1} \leq\left|M_{1}\right| \leq c^{\prime}\left(|\lambda|^{1 / 2}+A\right) \quad\left(M_{1}=A_{+}, B_{ \pm}\right)
$$

for any $\left(\lambda, \xi^{\prime}\right) \in \tilde{\Gamma}_{\epsilon, \lambda_{0}}=\Gamma_{\epsilon, \lambda_{0}} \times\left(\mathbb{R}^{N-1} \backslash\{0\}\right)$ with some positive constants $c$ and $c^{\prime}$, which immediately follows from Lemma 4.1. Here and in the following, $c$ and $c^{\prime}$ denote some positive constants essentially depending on $\mu_{ \pm}, v_{+}, \gamma_{0 \pm}, \gamma_{+1}, \gamma_{+2}, \epsilon, \lambda_{0}$ and $\delta_{0}$. In particular, by (4.1) we have

$$
c\left(|\lambda|^{1 / 2}+A\right) \leq \operatorname{Re} M_{2} \leq\left|M_{2}\right| \leq c^{\prime}\left(|\lambda|^{1 / 2}+A\right) \quad\left(M_{2}=A_{+}+B_{+}, \mu_{+} B_{+}+\mu_{-} B_{-}\right)
$$


for any $\left(\lambda, \xi^{\prime}\right) \in \tilde{\Gamma}_{\epsilon, \lambda_{0}}$. As was shown in Enomoto and Shibata [17, Lemma 4.3], using (4.1), (4.2), and the Bell formula (3.3), we see that

$$
\left(M_{3}\right)^{s} \in \mathbf{M}_{s, 1} \quad\left(M_{3}=A_{+}, B_{+}, A_{+}+B_{+}, \mu_{+} B_{+}+\mu_{-} B_{-}\right) .
$$

Especially, we have (2.29).

Second, we estimate $\left(A_{+} B_{+}-A^{2}\right)^{-1}$. For this purpose, we write

$$
\begin{gathered}
\frac{1}{A_{+} B_{+}-A^{2}}=\frac{\left(\mu_{+}+v_{+}+\delta\right) \mu_{+}}{\gamma_{0+}\left(2 \mu_{+}+v_{+}+\delta\right) \lambda} P\left(\lambda, \xi^{\prime}\right) \\
\quad \text { with } P\left(\lambda, \xi^{\prime}\right)=\frac{A_{+} B_{+}+A^{2}}{\gamma_{0+}\left(2 \mu_{+}+\nu_{+}+\delta\right)^{-1} \lambda+A^{2}} .
\end{gathered}
$$

By Lemma 4.1, (3.3), and (4.3) we have

$$
A_{+} B_{+}+A^{2} \in \mathbf{M}_{2,1}, \quad\left(\gamma_{0+}\left(2 \mu_{+}+\mu_{+}+\delta\right)^{-1} \lambda+A^{2}\right)^{s} \in \mathbf{M}_{2 s, 1},
$$

so that by Lemma 2.2 we have

$$
P \in \mathbf{M}_{0,1} \text {. }
$$

Since $A^{2}-A_{+}^{2}=\gamma_{0+}\left(\mu_{+}+v_{+}+\delta\right)^{-1} \lambda$, by (4.4) and (4.6), we have $\frac{A^{2}-A_{+}^{2}}{A_{+} B_{+}-A^{2}} \in \mathbf{M}_{0,1}$, which, combined with (4.3), furnishes (2.25).

Applying (4.4) to the formula in (2.15), we have

$$
\begin{aligned}
& L_{11}^{+}=\frac{\mu_{+}\left(\mu_{+}+v_{+}+\delta\right)}{2 \mu_{+}+v_{+}+\delta} A_{+} P, \quad L_{12}^{+}=\mu_{+} A\left(2-\frac{\mu_{+}+v_{+}+\delta}{2 \mu_{+}+v_{+}+\delta} P\right), \\
& L_{21}^{+}=\left(\frac{2 \mu_{+}\left(v_{+}+\delta\right)}{2 \mu_{+}+v_{+}+\delta} \frac{A_{+}}{B_{+}+A_{+}}-\frac{\mu_{+}\left(v_{+}-\mu_{+}+\delta\right)}{2 \mu_{+}+v_{+}+\delta}\right) A P, \\
& L_{22}^{+}=\frac{\mu_{+}\left(\mu_{+}+v_{+}+\delta\right)}{2 \mu_{+}+v_{+}+\delta} B_{+} P .
\end{aligned}
$$

Noting that $A \in \mathbf{M}_{1,2}$, by Lemma 2.2, (2.15), (4.3), (4.6), and (4.7), we have $L_{11}^{+} \in \mathbf{M}_{1,1}, L_{12}^{+} \in$ $\mathbf{M}_{1,2}, L_{21}^{+} \in \mathbf{M}_{1,2}$, and $L_{22}^{+} \in \mathbf{M}_{1,1}$. In addition, since $A \in \mathbf{M}_{1,2}$ and $B_{-} \in \mathbf{M}_{1,2}$, by Lemma 2.2 we have $A \pm B_{-} \in \mathbf{M}_{1,2}$ and $\left(A+B_{-}\right) B_{-} \in \mathbf{M}_{2,2}$. Summing up, we have proved (2.16).

\section{Analysis of Lopatinski determinant}

In this section, we show the following lemma, which implies (2.20).

Lemma 5.1 Let $L$ be the matrix defined in (2.17). Then there exists a positive constant $\omega$ depending solely on $\mu_{ \pm}, \nu_{+}, \epsilon, \gamma_{0 \pm}, \gamma_{1_{+}}, \gamma_{2+}, \lambda_{0}$, and $\delta_{0}$ such that

$$
|\operatorname{det} L| \geq \omega\left(|\lambda|^{1 / 2}+A\right)^{3}
$$

for any $\left(\lambda, \xi^{\prime}\right) \in \tilde{\Gamma}_{\epsilon, \lambda}$.

Moreover, we have

$$
\left|\partial_{\xi^{\prime}}^{\kappa^{\prime}}\left\{\left(\tau \partial_{\tau}\right)^{\ell}(\operatorname{det} L)^{-1}\right\}\right| \leq C_{\kappa^{\prime}}\left(|\lambda|^{1 / 2}+A\right)^{-3} A^{-\left|\kappa^{\prime}\right|} \quad(\ell=0,1)
$$

for any multi-index $\kappa^{\prime} \in \mathbb{N}_{0}^{N-1}$ and $\left(\lambda, \xi^{\prime}\right) \in \tilde{\Gamma}_{\epsilon, \lambda}$. Namely, $(\operatorname{det} L)^{-1} \in \mathbf{M}_{-3,2}$. 
Proof Recalling (1.13) and setting $\delta_{2}=\max \left(\delta_{0}, \gamma_{1+} \gamma_{2+} \lambda_{0}^{-1}\right)$, we have $\delta \in \Sigma_{\epsilon}$ and $|\delta| \leq \delta_{2}$. Moreover, by Lemma 4.1

$$
\left(\sin \frac{\sigma}{2}\right)\left(s \mu_{+}+v_{+}\right) \leq\left|s \mu_{+}+v_{+}+\delta\right| \leq s \mu_{+}+v_{+}+\delta_{2}
$$

with $s=0,1,2$. To prove (5.1), first we consider the case $R_{1}|\lambda|^{1 / 2} \leq A$ with large $R_{1} \geq 1$. Let $P$ be the function defined in (4.4). By (4.4) we see easily that $P=2+O\left(\delta_{3}\right)$, that $A_{+}=$ $A\left(1+O\left(\delta_{3}\right)\right)$, and that $B_{ \pm}=A\left(1+O\left(\delta_{3}\right)\right)$ when $\left|\gamma_{0_{+}}\left(\mu_{+}+\nu_{+}+\delta\right)^{-1} \lambda A^{-2}\right| \leq \gamma_{0_{+}}\left(\left(\sin \frac{\sigma}{2}\right)\left(\mu_{+}^{1}+\right.\right.$ $\left.\left.\nu_{+}\right) R_{1}^{2}\right)^{-1} \leq \delta_{3}$ and $\left|\gamma_{0 \pm}\left(\mu_{ \pm}\right)^{-1} \lambda A^{-2}\right| \leq \gamma_{0 \pm}\left(\mu_{ \pm} R_{1}^{2}\right)^{-1} \leq \delta_{3}$ with very small positive number $\delta_{3}$. Thus, by (4.7) we have

$$
\begin{aligned}
& L_{11}^{+}=\frac{2 \mu_{+}\left(\mu_{+}+v_{+}+\delta\right)}{2 \mu_{+}+v_{+}+\delta} A\left(1+O\left(\delta_{3}\right)\right), \quad A L_{12}^{+}=\frac{2\left(\mu_{+}\right)^{2}}{2 \mu_{+}+v_{+}+\delta} A^{2}\left(1+O\left(\delta_{3}\right)\right), \\
& L_{21}^{+}=\frac{2\left(\mu_{+}\right)^{2}}{2 \mu_{+}+v_{+}+\delta} A\left(1+O\left(\delta_{3}\right)\right), \quad A L_{22}^{+}=\frac{2 \mu_{+}\left(\mu_{+}+v_{+}+\delta\right)}{2 \mu_{+}+v_{+}+\delta} A^{2}\left(1+O\left(\delta_{3}\right)\right) \text {. }
\end{aligned}
$$

On the other hand, we have $B_{-}-A=\frac{\gamma_{0-\lambda}}{\mu_{0-}\left(B_{-}+A\right)}=A O\left(\delta_{3}\right)$, so that by $(2.15)$ we have

$$
\begin{aligned}
& L_{11}^{-}=2 \mu_{-} A\left(1+O\left(\delta_{3}\right)\right), \quad A L_{12}^{-}=A^{2} O\left(\delta_{3}\right), \\
& L_{21}^{-}=A O\left(\delta_{3}\right), \quad L_{22}^{-}=2 \mu_{-} A^{2}\left(1+O\left(\delta_{3}\right)\right) .
\end{aligned}
$$

Summing up, we have

$$
\begin{aligned}
& L_{11}=\left(\frac{2 \mu_{+}\left(\mu_{+}+v_{+}+\delta\right)}{2 \mu_{+}+v_{+}+\delta}+2 \mu_{-}\right) A\left(1+O\left(\delta_{3}\right)\right), \\
& A L_{12}=\frac{2\left(\mu_{+}\right)^{2}}{2 \mu_{+}+v_{+}+\delta} A^{2}\left(1+O\left(\delta_{3}\right)\right), \quad L_{21}=\frac{2\left(\mu_{+}\right)^{2}}{2 \mu_{+}+v_{+}+\delta} A\left(1+O\left(\delta_{3}\right)\right), \\
& L_{22}=\left(\frac{2 \mu_{+}\left(\mu_{+}+v_{+}+\delta\right)}{2 \mu_{+}+v_{+}+\delta}+2 \mu_{-}\right) A^{2}\left(1+O\left(\delta_{3}\right)\right),
\end{aligned}
$$

so that we have

$$
\begin{aligned}
\operatorname{det} L & =\left\{\left(\frac{2 \mu_{+}\left(\mu_{+}+v_{+}+\delta\right)}{2 \mu_{+}+v_{+}+\delta}+2 \mu_{-}\right)^{2}-\left(\frac{2\left(\mu_{+}\right)^{2}}{2 \mu_{+}+v_{+}+\delta}\right)^{2}\right\} A^{3}\left(1+O\left(\delta_{3}\right)\right) \\
& =4\left(\mu_{+}+\mu_{-}\right)\left(\frac{\mu_{+}\left(v_{+}+\delta\right)}{2 \mu_{+}+v_{+}+\delta}+\mu_{-}\right) A^{3}\left(1+O\left(\delta_{3}\right)\right) .
\end{aligned}
$$

Since $\frac{\mu_{+}\left(v_{+}+\delta\right)}{2 \mu_{+}+v_{+}+\delta}+\mu_{-}=1+O\left(|\delta|^{-1}\right)$ as $|\delta| \rightarrow \infty$, we have

$$
\left|\frac{\mu_{+}\left(v_{+}+\delta\right)}{2 \mu_{+}+v_{+}+\delta}+\mu_{-}\right| \geq \frac{1}{2}+\mu_{-} \quad \text { when }|\delta| \geq K_{0}
$$

with some large number $K_{0}$ depending on $\mu_{+}$and $\nu_{+}$. On the other hand, when $\delta \in \Sigma_{\epsilon}$ and $|\delta| \leq K_{0}$, we write

$$
\frac{\mu_{+}\left(\mu_{+}+v_{+}+\delta\right)}{2 \mu_{+}+v_{+}+\delta}+\mu_{-}=\frac{\mu_{+} \mu_{-}+\left(\mu_{+}+\mu_{-}\right)\left(\mu_{+}+v_{+}\right)+\delta\left(\mu_{+}+\mu_{-}\right)}{2 \mu_{+}+v_{+}+\delta} .
$$


Since $\mu_{ \pm}>0$ and $\nu_{+}>0$ and $\delta \in \Sigma_{\epsilon}$, by Lemma $4.1(1)$

$$
\begin{aligned}
& \left|\mu_{+} \mu_{-}+\left(\mu_{+}+\mu_{-}\right)\left(\mu_{+}+v_{+}\right)+\delta\left(\mu_{+}+\mu_{-}\right)\right| \\
& \geq\left(\sin \frac{\epsilon}{2}\right)\left(|\delta|\left(\mu_{+}+\mu_{-}\right)+\mu_{+} \mu_{-}+\left(\mu_{+}+\mu_{-}\right)\left(\mu_{+}+v_{+}\right)\right) \\
& \quad \geq\left(\sin \frac{\epsilon}{2}\right)\left(\mu_{+} \mu_{-}+\left(\mu_{+}+\mu_{-}\right)\left(\mu_{+}+v_{+}\right)\right)
\end{aligned}
$$

On the other hand, $\left|2 \mu_{+}+v_{+}+\delta\right| \leq 2 \mu_{+}+\nu_{+}+\delta_{2}$, so that we have

$$
|\operatorname{det} L| \geq 2\left(\mu_{+}+\mu_{-}\right) \min \left\{\left(\sin \frac{\epsilon}{2}\right) \frac{\left(\mu_{+} \mu_{-}+\left(\mu_{+}+\mu_{-}\right)\left(\mu_{+}+v_{+}\right)\right)}{2 \mu_{+}+v_{+}+\delta_{2}}, \frac{1}{2}+\mu_{-}\right\} A^{3}
$$

provided that $A \geq|\lambda|^{1 / 2} R_{1}$ with some constant $R_{1} \geq 1$ depending on $\mu_{ \pm}, \nu_{+}, \gamma_{0 \pm}, \gamma_{+1}, \gamma_{2+}$, $\epsilon, \lambda_{0}$ and $\delta_{0}$, which furnishes (5.1) when $A \geq|\lambda|^{1 / 2} R_{1}$ with any constant $\omega$ satisfying

$$
0<\omega \leq 2\left(\mu_{+}+\mu_{-}\right) \min \left\{\left(\sin \frac{\epsilon}{2}\right) \frac{\left(\mu_{+} \mu_{-}+\left(\mu_{+}+\mu_{-}\right)\left(\mu_{+}+v_{+}\right)\right)}{2 \mu_{+}+v_{+}+\delta_{2}}, \frac{1}{2}+\mu_{-}\right\} .
$$

Secondly, we consider the case $R_{2} A \leq|\lambda|^{1 / 2}$ with large $R_{2} \geq 1$. In this case, we have

$$
A_{+}=\left(\mu_{+}+\nu_{+}+\delta\right)^{-1 / 2}\left(\gamma_{0+} \lambda\right)^{1 / 2}\left(1+O\left(\delta_{4}\right)\right), \quad B_{ \pm}=\left(\mu_{ \pm}\right)^{-1 / 2}\left(\gamma_{0 \pm} \lambda\right)^{1 / 2}\left(1+O\left(\delta_{4}\right)\right)
$$

when $\left|\left(\mu_{+}+v_{+}+\delta\right)\left(\gamma_{0+} \lambda\right)^{-1} A^{2}\right| \leq \gamma_{0+}^{-1}\left(\mu_{+}+\nu_{+}+\delta_{1}\right) R_{2}^{-2} \leq \delta_{4}$ and $\left|\mu_{ \pm}\left(\gamma_{0 \pm} \lambda\right)^{-1} A^{2}\right| \leq$ $\gamma_{0 \pm}^{-1} \mu_{ \pm} R_{2}^{-2} \leq \delta_{4}$ with some very small positive number $\delta_{4}$. By (2.15)

$$
\begin{aligned}
& L_{11}=\left(\left(\mu_{+} \gamma_{0_{+}}\right)^{1 / 2}+\left(\mu_{-} \gamma_{0-}\right)^{1 / 2}\right) \lambda^{1 / 2}\left(1+O\left(\delta_{4}\right)\right), \quad A L_{12}=\lambda O\left(R_{2}^{-1}\right)\left(1+O\left(\delta_{4}\right)\right), \\
& L_{21}=\left(\mu_{-}\right)^{1 / 2}\left(\gamma_{0-} \lambda\right)^{1 / 2}\left(1+O\left(\delta_{4}\right)\right), \quad L_{22}=\gamma_{0-} \lambda\left(1+O\left(\delta_{4}\right)\right) .
\end{aligned}
$$

Thus, we have

$$
|\operatorname{det} L| \geq \frac{\gamma_{0-}}{2}\left(\left(\mu_{+} \gamma_{0_{+}}\right)^{1 / 2}+\left(\mu_{-} \gamma_{0_{-}}\right)^{1 / 2}\right)|\lambda|^{3 / 2}
$$

provided that $R_{2} A \leq|\lambda|^{1 / 2}$ with some constant $R_{2} \geq 1$ depending on $\mu_{ \pm}, \nu_{+}, \gamma_{0 \pm}, \gamma_{1+}, \gamma_{2+}$, $\epsilon, \lambda_{0}$, and $\delta_{0}$, which shows that (5.1) holds when $R_{2} A \leq|\lambda|^{1 / 2}$ with any constant $\omega$ satisfying

$$
0<\omega \leq \frac{\gamma_{0-}}{2}\left(\left(\mu_{+} \gamma_{0_{+}}\right)^{1 / 2}+\left(\mu_{-} \gamma_{0_{-}}\right)^{1 / 2}\right)
$$

Thirdly, we consider the case $R_{2}^{-1}|\lambda|^{1 / 2} \leq A \leq R_{1}|\lambda|^{1 / 2}$. Set

$$
\begin{aligned}
& \tilde{\lambda}=\frac{\lambda}{\left(|\lambda|^{1 / 2}+A\right)^{2}}, \quad \tilde{A}=\frac{A}{|\lambda|^{1 / 2}+A}, \\
& \tilde{A}_{+}=\sqrt{\gamma_{0+}\left(\mu_{+}+v_{+}+\delta\right)^{-1} \tilde{\lambda}+\tilde{A}^{2}}, \quad \tilde{B}_{ \pm}=\sqrt{\gamma_{0 \pm}\left(\mu_{ \pm}^{1}\right)^{-1} \tilde{\lambda}+\tilde{A}^{2}}, \\
& D\left(R_{1}, R_{2}\right)=\left\{(\tilde{\lambda}, \tilde{A})\left|\left(1+R_{1}\right)^{-2} \leq\right| \tilde{\lambda} \mid \leq R_{2}^{2}\left(1+R_{2}\right)^{2},\left(1+R_{2}\right)^{-1} \leq \tilde{A} \leq R_{1}\left(1+R_{1}\right)^{-1}\right\} .
\end{aligned}
$$


If $\left(\lambda, \xi^{\prime}\right)$ satisfies the condition $R_{2}^{-1}|\lambda|^{1 / 2} \leq A \leq R_{1}|\lambda|^{1 / 2}$, then $(\tilde{\lambda}, \tilde{A}) \in D\left(R_{1}, R_{2}\right)$. We define $\tilde{L}_{i j}$ by replacing $A_{+}, A$, and $B_{ \pm}$by $\tilde{A}_{+}, \tilde{A}$, and $\tilde{B}_{ \pm}$in (2.15), respectively. Setting $\operatorname{det} \tilde{L}=$ $\tilde{L}_{11} \tilde{L}_{22}-\tilde{A} \tilde{L}_{12} \tilde{L}_{21}$, we have

$$
\operatorname{det} L=\left(|\lambda|^{1 / 2}+A\right)^{3} \operatorname{det} \tilde{L}
$$

First, we prove that $\operatorname{det} \tilde{L} \neq 0$ provided that $(\tilde{\lambda}, \tilde{A}) \in D\left(R_{1}, R_{2}\right), \tilde{\lambda} \in \Sigma_{\epsilon}$ and $|\delta| \leq \delta_{5}$ with some small $\delta_{5}>0$ by contradiction. Suppose that $\operatorname{det} \tilde{L}=0$. By (5.6) $\operatorname{det} L=0$, so that in view of (2.18) there exist $w_{ \pm \ell}\left(x_{N}\right)=P_{ \pm \ell}\left(e^{\mp B_{ \pm} x_{N}}-e^{\mp A_{ \pm} x_{N}}\right)+Q_{ \pm \ell} e^{\mp B_{ \pm} x_{N}}$ and $p_{-}\left(x_{N}\right)=\gamma_{-} e^{A x_{N}}$ with $A_{-}=A$ such that $\mathbf{w}_{ \pm}\left(x_{N}\right)=\left(w_{ \pm 1}\left(x_{N}\right), \ldots, w_{ \pm N}\left(x_{N}\right)\right) \neq(0, \ldots, 0)$, and $\mathbf{w}_{ \pm}\left(x_{N}\right)$ and $p_{-}\left(x_{N}\right)$ satisfy $(2.2)$ and $(2.3)$ with $\hat{h}_{j}(0)=0, \hat{h}_{N}(0)=0$, and $\hat{k}_{J}(0)=0$, that is, they satisfy the following homogeneous equations:

$$
\begin{aligned}
& \gamma_{0+} \lambda w_{+j}-\sum_{\ell=1}^{N-1} \mu_{+} i \xi_{\ell}\left(i \xi_{j} w_{+\ell}+i \xi_{\ell} w_{+j}\right)-\mu_{+} \partial_{N}\left(i \xi_{j} w_{+N}+\partial_{N} w_{+j}\right) \\
& \quad-\left(v_{+}-\mu_{+}+\delta\right) i \xi_{j}\left(i \xi^{\prime} \cdot w_{+}^{\prime}+\partial_{N} w_{+N}\right)=0 \quad \text { for } x_{N}>0, \\
& \gamma_{0+} \lambda w_{+N}-\sum_{\ell=1}^{N-1} \mu_{+} i \xi_{\ell}\left(\partial_{N} w_{+\ell}+i \xi_{\ell} w_{+N}\right)-2 \mu_{+} \partial_{N}^{2} w_{+N} \\
& \quad-\left(v_{+}-\mu_{+}+\delta\right) \partial_{N}\left(i \xi^{\prime} \cdot w_{+}^{\prime}+\partial_{N} w_{+N}\right)=0 \quad \text { for } x_{N}>0, \\
& \gamma_{0-} \lambda w_{-j}-\sum_{\ell=1}^{N-1} \mu_{-} i \xi_{\ell}\left(i \xi_{j} w_{-\ell}+i \xi_{\ell} w_{-j}\right) \\
& \quad-\mu_{-} \partial_{N}\left(i \xi_{j} w_{-N}+\partial_{N} w_{j}\right)+i \xi_{j} p_{-}=0 \quad \text { for } x_{N}<0, \\
& \gamma_{0-} \lambda w_{-N}-\sum_{\ell=1}^{N-1} \mu_{-} i \xi_{\ell}\left(\partial_{N} w_{-\ell}+i \xi_{\ell} w_{-N}\right)-2 \mu_{-} \partial_{N}^{2} w_{-N}+\partial_{N} p_{-}=0 \quad \text { for } x_{N}<0, \\
& i \xi^{\prime} \cdot w_{-}^{\prime}+\partial_{N} w_{-N}=0 \quad \text { for } x_{N}<0, \\
& \left.\mu_{+}\left(\partial_{N} w_{+j}+i \xi_{j} w_{+N}\right)\right|_{x_{N}=0+}-\left.\mu_{-}\left(\partial_{N} w_{-j}+i \xi_{j} w_{-}\right)\right|_{x_{N}=0-}=0, \\
& 2 \mu_{+} \partial_{N} w_{+N}+\left.\left(v_{+}-\mu_{+}+\delta\right)\left(i \xi^{\prime} \cdot w_{+}^{\prime}+\partial_{N} w_{+N}\right)\right|_{x_{N}=0+}-\left.\left(2 \mu_{-} \partial_{N} w_{-N}-p_{-}\right)\right|_{x_{N}=0-}=0 .
\end{aligned}
$$

Set $(a, b)_{+}=\int_{0}^{\infty} a\left(x_{N}\right) \overline{b\left(x_{N}\right)} d x_{N},(a, b)_{-}=\int_{-\infty}^{0} a\left(x_{N}\right) \overline{b_{N}\left(x_{N}\right)} d x_{N}$, and $\|a\|_{ \pm}=(a, a)_{ \pm}^{1 / 2}$. Multiplying the equations in (5.7) by $\overline{w_{ \pm J}}$ and using integration by parts and the jump conditions in (5.7), we have

$$
\begin{aligned}
0= & \lambda\left(\gamma_{0+} \sum_{\ell=1}^{N}\left\|w_{+\ell}\right\|^{2}+\gamma_{0-} \sum_{\ell=1}^{N}\left\|w_{-\ell}\right\|^{2}\right) \\
& +\mu_{+}\left[\sum_{j, k=1}^{N-1}\left\|i \xi_{k} w_{+j}\right\|_{+}^{2}+\left\|i \xi^{\prime} \cdot w_{+}^{\prime}\right\|_{+}^{2}+\sum_{j=1}^{N-1}\left\|\partial_{N} w_{+j}\right\|_{+}^{2}\right. \\
& \left.+\sum_{j=1}^{N-1}\left(i \xi_{j} w_{+N}, \partial_{N} w_{+N}\right)_{+}+\sum_{j=1}^{N-1}\left\|i \xi_{j} w_{+N}\right\|_{+}^{2}+\sum_{j=1}^{N-1}\left(\partial_{N} w_{+j}, i \xi_{j} w_{+N}\right)_{+}+2\left\|\partial_{N} w_{+N}\right\|_{+}^{2}\right] \\
& +\left(v_{+}-\mu_{+}+\delta\right)\left[\left\|i \xi^{\prime} \cdot w_{+}^{\prime}\right\|_{+}^{2}+\left(\partial_{N} w_{+N}, i \xi^{\prime} \cdot w_{+}^{\prime}\right)_{+}+\left(i \xi^{\prime} \cdot w_{+}^{\prime}, \partial_{N} w_{+N}\right)_{+}+\left\|\partial_{N} w_{+N}\right\|_{+}^{2}\right]
\end{aligned}
$$




$$
\begin{aligned}
& +\mu_{-}\left[\sum_{j, k=1}^{N-1}\left\|i \xi_{k} w_{-j}\right\|_{-}^{2}+\left\|i \xi^{\prime} \cdot w_{-}^{\prime}\right\|_{-}^{2}+\sum_{j=1}^{N-1}\left\|\partial_{N} w_{-j}\right\|_{-}^{2}\right. \\
& \left.+\sum_{j=1}^{N-1}\left(i \xi_{j} w_{-N}, \partial_{N} w_{-N}\right)_{-}+\sum_{j=1}^{N-1}\left\|i \xi_{j} w_{-N}\right\|_{-}^{2}+\sum_{j=1}^{N-1}\left(\partial_{N} w_{-j}, i \xi_{j} w_{-N}\right)_{-}+2\left\|\partial_{N} w_{-N}\right\|_{-}^{2}\right] \\
= & \lambda\left(\gamma_{0+}\left\|w_{+}\right\|_{+}^{2}+\gamma_{0-}\left\|w_{-}\right\|_{-}^{2}\right)+\mu_{+}\left[\sum_{j, k=1}^{N-1}\left\|i \xi_{k} w_{+j}\right\|_{+}^{2}+\left\|i \xi^{\prime} \cdot w_{+}^{\prime}\right\|_{+}^{2}\right. \\
& \left.+\sum_{j=1}^{N-1}\left\|\partial_{N} w_{+j}+i \xi_{j} w_{+N}\right\|_{+}^{2}+2\left\|\partial_{N} w_{+N}\right\|_{+}^{2}\right]+\left(v_{+}-\mu_{+}+\delta\right)\left\|\partial_{N} w_{+N}+i \xi^{\prime} \cdot w_{+}^{\prime}\right\|_{+}^{2} \\
& +\mu_{-}\left[\sum_{j, k=1}^{N-1}\left\|i \xi_{k} w_{-j}\right\|_{-}^{2}+\left\|i \xi^{\prime} \cdot w_{-}^{\prime}\right\|_{-}^{2}+\sum_{j=1}^{N-1}\left\|\partial_{N} w_{-j}+i \xi_{j} w_{-N}\right\|_{-}^{2}+2\left\|\partial_{N} w_{-N}\right\|_{-}^{2}\right] .
\end{aligned}
$$

Taking the real part and the imaginary part in (5.8), using the inequality

$$
\begin{aligned}
& \sum_{j, k=1}^{N-1}\left\|i \xi_{j} w_{+k}\right\|_{+}^{2}+\left\|i \xi^{\prime} \cdot w_{+}^{\prime}\right\|_{+}^{2}+2\left\|\partial_{N} w_{+N}\right\|_{+}^{2} \\
& \quad \geq 2\left(\left\|i \xi^{\prime} \cdot w_{+}^{\prime}\right\|_{+}^{2}+\left\|\partial_{N} w_{+N}\right\|_{+}^{2}\right) \geq\left\|\partial_{N} w_{+N}+i \xi^{\prime} \cdot w_{+}^{\prime}\right\|_{+}^{2},
\end{aligned}
$$

and setting $K=\gamma_{0+}\left\|\mathbf{w}_{+}\right\|_{+}^{2}+\gamma_{0-}\left\|\mathbf{w}_{-}\right\|_{-}^{2}$ and $L=\left\|\partial_{N} w_{+N}+i \xi^{\prime} \cdot w_{+}^{\prime}\right\|_{+}^{2}$ for short, we have

$$
(\operatorname{Im} \lambda) K+(\operatorname{Im} \delta) L=0, \quad 0 \geq(\operatorname{Re} \lambda) K+\left(v_{+}+\operatorname{Re} \delta\right) L .
$$

First, we consider the case $\delta=0$. When $\operatorname{Im} \lambda \neq 0$ or $\operatorname{Im} \lambda=0$ and $\operatorname{Re} \lambda>0$, we have $K=0$, that is, $\mathbf{w}_{ \pm}=0$. When $\operatorname{Im} \lambda=0$ and $\operatorname{Re} \lambda \leq 0$, it follows from $\lambda \in \Sigma_{\epsilon}$ that $\lambda=0$. Choosing $\epsilon^{\prime}>0$ in such a way that $\mu_{+}-\epsilon^{\prime}>0$ and $\nu_{+}-\epsilon^{\prime}>0$, by (5.8) with $\lambda=0$ and $\delta=0$ and (5.9) we have

$$
\begin{aligned}
\epsilon^{\prime} & {\left[\sum_{j, k=1}^{N-1}\left\|i \xi_{k} w_{+j}\right\|_{+}^{2}+\left\|i \xi^{\prime} \cdot w_{+}^{\prime}\right\|_{+}^{2}+2\left\|\partial_{N} w_{+N}\right\|_{+}^{2}\right]+\mu_{+} \sum_{j=1}^{N-1}\left\|\partial_{N} w_{+j}+i \xi_{j} w_{+N}\right\|_{+}^{2} } \\
& +\left(v_{+}-\epsilon^{\prime}\right)\left\|\partial_{N} w_{+N}+i \xi^{\prime} \cdot w_{+}^{\prime}\right\|_{+}^{2}+\mu_{-}\left[\sum_{j=1}^{N-1}\left\|\partial_{N} w_{-j}+i \xi_{j} w_{-N}\right\|_{-}^{2}+2\left\|\partial_{N} w_{-N}\right\|_{-}^{2}\right] \leq 0,
\end{aligned}
$$

which furnishes $\left\|\partial_{N} w_{ \pm j}+i \xi_{j} w_{ \pm N}\right\|_{ \pm}=0(j=1, \ldots, N-1)$ and $\left\|\partial_{N} w_{ \pm N}\right\|_{ \pm}=0$. Since $w_{ \pm J}\left(x_{N}\right) \rightarrow 0$ as $\pm x_{N} \rightarrow \infty(J=1, \ldots, N)$, we have $\mathbf{w}_{ \pm}=0$, which contradicts $\mathbf{w}_{ \pm} \neq 0$. Thus, we have $\operatorname{det} \tilde{L} \neq 0$ when $\delta=0$, which implies that

$$
c_{1}=\inf \left\{|\operatorname{det} \tilde{L}| \mid(\tilde{\lambda}, \tilde{A}) \in D\left(R_{1}, R_{2}\right), \tilde{\lambda} \in \Sigma_{\epsilon}, \delta=0\right\}>0 .
$$

Since $\tilde{A}_{+}=\sqrt{\gamma_{0+}\left(\mu_{+}+\nu_{+}\right)^{-1} \tilde{\lambda}+\tilde{A}^{2}}+O(|\delta|)$, there exists a $\delta_{5}>0$ such that

$$
\inf \left\{|\operatorname{det} \tilde{L}|\left|(\tilde{\lambda}, \tilde{A}) \in D\left(R_{1}, R_{2}\right), \tilde{\lambda} \in \Sigma_{\epsilon},\right| \delta \mid \leq \delta_{5}\right\} \geq c_{1} / 2
$$


which, combined with (5.6), implies that

$$
|\operatorname{det} L| \geq \omega_{1}\left(|\lambda|^{1 / 2}+A\right)^{3}
$$

with some positive number $\omega_{1}$ provide that $R_{2}|\lambda|^{1 / 2} \leq A \leq R_{1}^{-1}|\lambda|^{1 / 2}$ and $\lambda \in \mathbb{C}$ with $|\delta| \leq \delta_{5}$.

Finally, we consider the case where $|\delta| \geq \delta_{5}$. First, we consider the case (C1), that is, $\delta=\gamma_{1+} \gamma_{2+} \lambda^{-1}$. In this case, it follows from $|\delta| \geq \delta_{5}$ that $|\lambda| \leq \gamma_{1+} \gamma_{2+} \delta_{5}^{-1}$, so that we prove that $\operatorname{det} L \neq 0$ directly provided that $\lambda \in \Lambda_{\epsilon, \lambda_{0}}=\Sigma_{\epsilon, \lambda_{0}} \cap K_{\epsilon}$. Since $\operatorname{Re} \delta=\gamma_{1+} \gamma_{2+} \operatorname{Re} \lambda|\lambda|^{-2}$ and $\operatorname{Im} \delta=-\gamma_{1+} \gamma_{2+} \operatorname{Im} \lambda|\lambda|^{-2}$, by (5.10) we have

$$
\operatorname{Im} \lambda\left(K-\gamma_{1+} \gamma_{2+}|\lambda|^{-2} L\right)=0, \quad 0 \geq(\operatorname{Re} \lambda) K+\left(\nu_{+}+\gamma_{1+} \gamma_{2+} \operatorname{Re} \lambda|\lambda|^{-2}\right) L
$$

When $\operatorname{Im} \lambda=0$, we have $\lambda_{0} \leq \operatorname{Re} \lambda=\lambda$, so that $K=0$, that is, $\mathbf{w}_{ \pm}=0$. If $\operatorname{Im} \lambda \neq 0$, by (5.12) $K=\gamma_{1+} \gamma_{2+}|\lambda|^{-2} L$, which, inserted into the second formula in (5.12), furnishes

$$
\begin{aligned}
0 & \geq v_{+}|\lambda|^{-2}\left(|\lambda|^{2}+2 \gamma_{1+} \gamma_{2+} \nu_{+}^{-1} \operatorname{Re} \lambda\right) L \\
& =v_{+}|\lambda|^{-2}\left(\left(\operatorname{Re} \lambda+\gamma_{+1} \gamma_{+2} v_{+}^{-1}\right)^{2}+(\operatorname{Im} \lambda)^{2}-\left(\gamma_{1+} \gamma_{2+} \nu_{+}^{-1}\right)^{2}\right) L .
\end{aligned}
$$

Since $\left(\operatorname{Re} \lambda+\gamma_{+1} \gamma_{+2} v_{+}^{-1}\right)^{2}+(\operatorname{Im} \lambda)^{2}-\left(\gamma_{1+} \gamma_{2+} \nu_{+}^{-1}\right)^{2}>0$ when $\lambda \in K_{\epsilon}$, we have $L=0$, which implies that $K=0$, that is, $\mathbf{w}_{ \pm}=0$. Summing up, we have obtained $\mathbf{w}_{ \pm}=0$, which contradicts $\mathbf{w}_{ \pm} \neq 0$, and therefore we have $\operatorname{det} L \neq 0$ when $\lambda \in \Sigma_{\epsilon, \lambda_{0}} \cap K_{\epsilon}$. Thus, we have

$$
\inf \left\{\left.|\operatorname{det} L|\left|\lambda \in \Sigma_{\epsilon, \lambda_{0}} \cap K_{\epsilon}, R_{2}^{-1}\right| \lambda\right|^{1 / 2} \leq A \leq R_{1}|\lambda|^{1 / 2},|\lambda| \leq \gamma_{1+} \gamma_{2+} \delta_{5}^{-1}\right\}>0 \text {, }
$$

which, combined with (5.11), furnishes

$$
|\operatorname{det} L| \geq \omega_{2}\left(|\lambda|^{1 / 2}+A\right)^{3}
$$

with some positive constant $\omega_{2}$ provided that $R_{2}^{-1}|\lambda|^{1 / 2} \leq A \leq R_{1}|\lambda|^{1 / 2}$ and $\lambda \in K_{\epsilon} \cap \Sigma_{\epsilon, \lambda_{0}}$.

Secondly, we consider the case where $\delta \in \Sigma_{\epsilon}, \delta_{5} \leq|\delta| \leq \delta_{0}, \operatorname{Re} \delta \leq 0,|\lambda| \geq \lambda_{0}$ and $\operatorname{Re} \lambda \geq$ $|\operatorname{Re} \delta / \operatorname{Im} \delta||\operatorname{Im} \lambda|$. Note that this case includes (C2) and $\operatorname{Im} \delta \neq 0$. We prove that $\operatorname{det} \tilde{L} \neq 0$ provided that $(\tilde{\lambda}, \tilde{A}) \in D\left(R_{1}, R_{2}\right)$ and $\operatorname{Re} \tilde{\lambda} \geq|\operatorname{Re} \delta / \operatorname{Im} \delta||\operatorname{Im} \tilde{\lambda}|$ by contradiction. Suppose that $\operatorname{det} \tilde{L}=0$, and then by (5.6), $\operatorname{det} L=0$. Thus, we have (5.10). When $\operatorname{Im} \lambda=0$ and $\operatorname{Im} \delta \neq$ 0 , we have $L=0$, so that $0 \geq(\operatorname{Re} \lambda) K$. When $\operatorname{Re} \lambda>0$, we have $K=0$. When $\lambda=0$, by (5.8) with $\lambda=0$ and $L=0$, we have $\left\|\partial_{N} w_{ \pm N}\right\|_{ \pm}=0$ and $\left\|\partial_{N} w_{ \pm j}+i \xi_{j} w_{ \pm N}\right\|_{ \pm}^{2}=0$. Since $w_{ \pm J}\left(x_{N}\right) \rightarrow 0$ as $\pm x_{N} \rightarrow \infty$, we have $\mathbf{w}_{ \pm}=0$. Thus, we have $\mathbf{w}_{ \pm}=0$ when $\operatorname{Im} \lambda=0$ and $\operatorname{Im} \delta \neq 0$. When $\operatorname{Im} \lambda \neq 0, \operatorname{Im} \delta \neq 0$ and $(\operatorname{Im} \lambda) \operatorname{Im} \delta>0$, we have $K=0$ by the first formula of (5.10). When $\operatorname{Im} \lambda \neq 0, \operatorname{Im} \delta \neq 0$ and $(\operatorname{Im} \lambda) \operatorname{Im} \delta<0$, we have $K=|\operatorname{Im} \delta / \operatorname{Im} \lambda| L$ by the first formula of (5.10), so that it follows from the second formula of (5.10) that $0 \geq\left(v_{+}+\operatorname{Re} \delta+\right.$ $(\operatorname{Re} \lambda)|\operatorname{Im} \delta / \operatorname{Im} \lambda|) L$. Since $\operatorname{Re} \delta=-|\operatorname{Re} \delta|$ and since $\operatorname{Re} \lambda \geq|\operatorname{Re} \delta / \operatorname{Im} \delta||\operatorname{Im} \lambda|$, as follows from $\operatorname{Re} \tilde{\lambda} \geq|\operatorname{Re} \delta / \operatorname{Im} \delta||\operatorname{Im} \tilde{\lambda}|$, we have $\operatorname{Re} \delta+(\operatorname{Re} \lambda)|\operatorname{Im} \delta / \operatorname{Im} \lambda| \geq-|\operatorname{Re} \delta|+|\operatorname{Re} \delta|=0$, which furnishes $L=0$. Thus, $K=0$. Summing up, we have proved that $K=0$, that is, $\mathbf{w}_{ \pm}=0$. But this contradicts $\mathbf{w}_{ \pm} \neq 0$, and therefore $\operatorname{det} \tilde{L} \neq 0$. In particular,

$$
\begin{gathered}
c_{2}=\inf \left\{|\operatorname{det} \tilde{L}|\left|(\tilde{\lambda}, \tilde{A}) \in D\left(R_{1}, R_{2}\right), \operatorname{Re} \tilde{\lambda} \geq\right| \operatorname{Re} \delta / \operatorname{Im} \delta|| \operatorname{Im} \tilde{\lambda} \mid,\right. \\
\left.\delta \in \Sigma_{\epsilon}, \operatorname{Re} \delta \leq 0, \delta_{5} \leq|\delta| \leq \delta_{0}\right\}>0,
\end{gathered}
$$


which, combined with (5.11) and (5.6), implies that

$$
|\operatorname{det} L| \geq \omega_{3}\left(|\lambda|^{1 / 2}+A\right)^{3}
$$

with some positive constant $\omega_{3}$ provided that $R_{2}|\lambda|^{1 / 2} \leq A \leq R_{1}^{-1}|\lambda|^{1 / 2}$ and $\delta \in \Sigma_{\epsilon}, \operatorname{Re} \delta<0$, and $\operatorname{Re} \lambda \geq|\operatorname{Re} \delta / \operatorname{Im} \delta||\operatorname{Im} \lambda|$.

Analogously, we have

$$
|\operatorname{det} L| \geq \omega_{4}\left(|\lambda|^{1 / 2}+A\right)^{3}
$$

with some positive constant $\omega_{4}$ provided that $R_{2}|\lambda|^{1 / 2} \leq A \leq R_{1}^{-1}|\lambda|^{1 / 2}$ and $\delta \in \Sigma_{\epsilon}, \operatorname{Re} \delta \geq 0$, and $\operatorname{Re} \lambda \geq \lambda_{0}|\operatorname{Im} \lambda|$. Therefore, we have proved (5.1).

Since

$$
\operatorname{det} L=L_{11} L_{22}-A L_{12} L_{21} \text {, }
$$

by (2.19), the Leibniz rule, the Bell formula (3.3) with $f(t)=1 / t, g\left(\xi^{\prime}\right)=\operatorname{det} L$, and (5.1), we have

$$
\left|\partial_{\xi^{\prime}}^{\kappa^{\prime}}(\operatorname{det} L)^{-1}\right| \leq C_{\kappa^{\prime}} \sum_{\ell=1}^{\left|\kappa^{\prime}\right|}|\operatorname{det} L|^{-(\ell+1)}\left(|\lambda|^{1 / 2}+A\right)^{3 \ell} A^{-\left|\kappa^{\prime}\right|} \leq C_{\kappa^{\prime}}\left(|\lambda|^{1 / 2}+A\right)^{-3} A^{-\left|\kappa^{\prime}\right|},
$$

which shows (5.2) with $\ell=0$. Analogously, we have (5.2) with $\ell=1$, which completes the proof of Lemma 5.1.

\section{Proofs of main results}

In this section, we prove Theorem 1.2. For this purpose, first of all we prove (2.38). For the multipliers appearing in $\mathcal{S}_{J 1}^{ \pm}(\lambda)$ of (2.36), by Lemma 2.2, (2.8), and (4.3) we have

$$
\begin{aligned}
& \frac{R_{J \ell,-1}^{+}}{A_{+}} \in \mathbf{M}_{-2,2}, \quad \frac{R_{J \ell, 0}^{+} \gamma_{0}^{ \pm} \lambda^{1 / 2}}{\mu_{+} B_{+}^{2} A_{+}} \in \mathbf{M}_{-2,2}, \quad \frac{R_{J \ell, 0}^{+}\left(i \xi_{m}\right)}{B_{+}^{2} A_{+}} \in \mathbf{M}_{-2,2}, \\
& R_{J \ell,-1}^{-} \in \mathbf{M}_{-1,2}, \quad \frac{R_{J \ell, 0}^{-} \gamma_{0}^{ \pm} \lambda^{1 / 2}}{\mu_{-} B_{-}^{2}} \in \mathbf{M}_{-1,2}, \quad \frac{R_{J \ell, 0}^{-}\left(i \xi_{m}\right)}{B_{-}^{2}} \in \mathbf{M}_{-1,2}, \\
& \frac{R_{J \ell,-1}^{ \pm} \gamma_{0}^{ \pm} \lambda^{1 / 2}}{\mu_{ \pm} B_{ \pm}^{2}} \in \mathbf{M}_{-2,2}, \quad \frac{R_{J \ell,-1}^{ \pm}\left(i \xi_{m}\right)}{B_{ \pm}^{2}} \in \mathbf{M}_{-2,2}, \quad \frac{R_{J \ell,-1}^{ \pm} \lambda^{1 / 2}}{B_{ \pm}^{2}} \in \mathbf{M}_{-2,2},
\end{aligned}
$$

so that by Lemma 3.1 with $K_{1}^{+}(\lambda)$ and $K_{2}^{+}(\lambda)$ and Lemma 3.2 with $K_{1}^{-}(\lambda)$ and $K_{2}^{-}(\lambda)$ we have

$$
\mathcal{R}_{\mathcal{L}\left(\mathcal{Z}\left(\mathbb{R}^{N}\right), L_{q}\left(\mathbb{R}_{ \pm}^{N}\right)^{2+N+N^{2}}\right)}\left(\left\{\left(\tau \partial_{\tau}\right)^{\ell} G_{\lambda} \mathcal{S}_{J 1}^{ \pm}(\lambda) \mid \lambda \in \Lambda_{\epsilon, \lambda_{0}}\right\}\right) \leq C \quad(\ell=0,1) .
$$

For the multipliers appearing in $\mathcal{S}_{J 2}^{ \pm}(\lambda)$ of (2.36), by Lemma 2.2, (2.8), and (4.3) we have

$$
\begin{aligned}
& S_{J \ell,-2}^{ \pm} \in \mathbf{M}_{-2,2}, \quad \frac{S_{J \ell,-1}^{ \pm} \gamma_{0}^{ \pm} \lambda^{1 / 2}}{\mu_{ \pm} B_{ \pm}^{2}} \in \mathbf{M}_{-2,2}, \\
& \frac{S_{J \ell,-1}^{ \pm}\left(i \xi_{m}\right)}{B \pm^{2}} \in \mathbf{M}_{-2,2}, \quad \frac{B_{ \pm} S_{J \ell,-1}^{ \pm} \gamma_{0}^{ \pm} \lambda^{1 / 2}}{\mu_{ \pm} B_{ \pm}^{2}} \in \mathbf{M}_{-2,2},
\end{aligned}
$$




$$
\begin{aligned}
& \frac{B_{ \pm} S_{J \ell,-2}^{ \pm}\left(i \xi_{m}\right)}{B_{ \pm}^{2}} \in \mathbf{M}_{-2,2}, \quad \frac{B_{ \pm} S_{J \ell,-1}^{ \pm} \gamma_{0}^{ \pm}}{\mu_{ \pm} B_{ \pm}^{2}} \in \mathbf{M}_{-2,2} \\
& \frac{B_{ \pm} S_{J \ell,-1}^{ \pm}}{B_{ \pm}^{2}} \in \mathbf{M}_{-2,2}
\end{aligned}
$$

so that by Lemma 3.1 with $K_{2}^{+}(\lambda)$ and Lemma 3.2 with $K_{2}^{-}(\lambda)$ we have

$$
\mathcal{R}_{\mathcal{L}\left(\mathcal{Z}\left(\mathbb{R}^{N}\right), L_{q}\left(\mathbb{R}_{ \pm}^{N}\right)^{\left.2+N+N^{2}\right)}\right.}\left(\left\{\left(\tau \partial_{\tau}\right)^{\ell} G_{\lambda} \mathcal{S}_{J 2}^{ \pm}(\lambda) \mid \lambda \in \Lambda_{\epsilon, \lambda_{0}}\right\}\right) \leq C \quad(\ell=0,1) .
$$

For the multipliers appearing in $\mathcal{S}_{J 3}^{ \pm}(\lambda)$ of (2.36), by Lemma 2.2, (2.8), and (4.3) we have

$$
\begin{aligned}
& T_{J,-1}^{ \pm} \in \mathbf{M}_{-1,1}, \quad \frac{T_{J, 0}^{ \pm} \gamma_{0}^{ \pm} \lambda^{1 / 2}}{\mu_{ \pm} B_{ \pm}^{2}} \in \mathbf{M}_{-1,1}, \quad \frac{T_{J, 0}^{ \pm}\left(i \xi_{m}\right)}{B_{ \pm}^{2}} \in \mathbf{M}_{-1,1}, \\
& \frac{B_{ \pm} T_{J,-1}^{ \pm} \gamma_{0}^{ \pm} \lambda^{1 / 2}}{\mu_{ \pm} B_{ \pm}^{2}} \in \mathbf{M}_{-1,1}, \quad \frac{B_{ \pm} T_{J, 0}^{ \pm} \gamma_{0}^{ \pm}}{\mu_{ \pm} B_{ \pm}^{2}} \in \mathbf{M}_{-1,1}, \quad \frac{B_{ \pm} T_{J, 0}^{ \pm}}{B_{ \pm}^{2}} \in \mathbf{M}_{-1,1},
\end{aligned}
$$

so that by Lemma 3.1 with $K_{4}^{+}(\lambda)$ and Lemma 3.2 with $K_{4}^{-}(\lambda)$ we have

$$
\mathcal{R}_{\mathcal{L}\left(\mathcal{Z}\left(\mathbb{R}^{N}\right), L_{q}\left(\mathbb{R}_{ \pm}^{N}\right)^{2+N+N^{2}}\right)}\left(\left\{\left(\tau \partial_{\tau}\right)^{\ell} G_{\lambda} \mathcal{S}_{J 3}^{ \pm}(\lambda) \mid \lambda \in \Lambda_{\epsilon, \lambda_{0}}\right\}\right) \leq C \quad(\ell=0,1)
$$

For the multipliers appearing in $\mathcal{S}_{J 4}^{+}(\lambda)$ of (2.36), by Lemma 2.2, (2.8), and (4.3) we have

$$
\begin{array}{ll}
\frac{U_{N, 0}^{+} \gamma_{0}^{+} \lambda^{1 / 2}}{\mu_{+} B_{+}^{2}} \in \mathbf{M}_{-1,1}, & \frac{U_{N, 0}^{+}\left(i \xi_{m}\right)}{B_{+}^{2}} \in \mathbf{M}_{-1,1}, \\
\frac{A_{+} U_{N, 0}^{+} \gamma_{0}^{+}}{\mu_{+} B_{+}^{2}} \in \mathbf{M}_{-1,1}, & \frac{A_{+} U_{N, 0}^{+}}{B_{+}^{2}} \in \mathbf{M}_{-1,1},
\end{array}
$$

so that by Lemma 3.1 with $K_{3}^{+}(\lambda)$ and $K_{4}^{+}(\lambda)$ we have

$$
\mathcal{R}_{\mathcal{L}\left(\mathcal{Z}\left(\mathbb{R}^{N}\right), L_{q}\left(\mathbb{R}_{+}^{N}\right)^{\left.2+N+N^{2}\right)}\right.}\left(\left\{\left(\tau \partial_{\tau}\right)^{\ell} G_{\lambda} \mathcal{S}_{J 4}^{+}(\lambda) \mid \lambda \in \Lambda_{\epsilon, \lambda_{0}}\right\}\right) \leq C \quad(\ell=0,1) .
$$

Finally, for the multipliers appearing in $\mathcal{P}_{-}(\lambda)$ of (2.36), by Lemma $2.2,(2.8)$, and (4.3) we have

$$
\begin{aligned}
& p_{\ell, 0}^{-} \frac{i \xi_{m}}{A} \in \mathbf{M}_{0,2}, \quad \frac{p_{\ell, 1}^{-} \gamma_{0}^{-} \lambda^{1 / 2}}{\mu_{-} B_{-}^{2}} \frac{i \xi_{j}}{A} \in \mathbf{M}_{0,2}, \quad \frac{p_{\ell, 1}^{-}\left(i \xi_{m}\right)}{B_{-}^{2}} \frac{i \xi_{j}}{A} \in \mathbf{M}_{0,2}, \\
& p_{\ell, 0}^{-} \in \mathbf{M}_{0,2}, \quad \frac{p_{\ell, 1}^{-} \gamma_{0}^{-} \lambda^{1 / 2}}{\mu_{-} B_{-}^{2}} \in \mathbf{M}_{0,2}, \quad \frac{p_{\ell, 1}^{-}\left(i \xi_{m}\right)}{B_{-}^{2}} \in \mathbf{M}_{0,2}
\end{aligned}
$$

so that by Lemma 3.5 with $L_{6}(\lambda)$ we have

$$
\mathcal{R}_{\mathcal{L}\left(\mathcal{Z}\left(\mathbb{R}^{N}\right), L_{q}\left(\mathbb{R}_{-}^{N}\right)^{N}\right)}\left(\left\{\left(\tau \partial_{\tau}\right)^{\ell} \nabla \mathcal{P}_{-}(\lambda) \mid \lambda \in \Lambda_{\epsilon, \lambda_{0}}\right\}\right) \leq C \quad(\ell=0,1) .
$$

Summing up, we have proved (2.38). 
To transfer the problem (1.10) to (2.1), we use the solutions $\mathbf{w}_{+}$and $\left(\mathbf{w}_{-}, \theta_{-}\right)$to the following equations:

$$
\begin{aligned}
& \begin{cases}\lambda \mathbf{w}_{+}-\gamma_{0+}^{-1} \operatorname{Div} \mathbf{S}_{\delta+}\left(\mathbf{w}_{+}\right)=\mathbf{f}_{+} & \text {in } \mathbb{R}_{+}^{N}, \\
\left.\mathbf{S}_{\delta+}\left(\mathbf{w}_{+}\right) \mathbf{n}\right|_{x_{N}=0+}=0 & \text { on } \mathbb{R}_{0}^{N},\end{cases}
\end{aligned}
$$

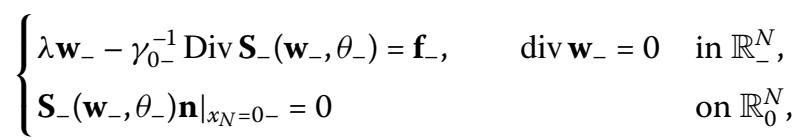

respectively. We know the following two theorems. The first theorem is due to Götz and Shibata [21, Theorem 2.5] and the second one is due to Shibata [18, Theorem 3.4].

Theorem 6.1 Let $1<q<\infty, 0<\epsilon<\pi / 2, \delta_{0}>0$ and $\lambda_{0}>0$. Let $\Gamma_{\epsilon, \lambda_{0}}$ be the set defined in (1.12). Then there exists an operator family $\mathcal{V}_{+}(\lambda) \in \operatorname{Hol}\left(\Gamma_{\epsilon, \lambda_{0}}, \mathcal{L}\left(L_{q}\left(\mathbb{R}_{+}^{N}\right)^{N}, W_{q}^{2}\left(\mathbb{R}_{+}^{N}\right)^{N}\right)\right)$ such that for any $\mathbf{f}_{+} \in L_{q}\left(\mathbb{R}_{+}^{N}\right)^{N}$ and $\lambda \in \Gamma_{\epsilon, \lambda_{0}}, \mathbf{w}_{+}=\mathcal{V}_{+}(\lambda) \mathbf{f}_{+}$is a unique solution to problem (6.1), and $\mathcal{V}_{+}(\lambda)$ satisfies the following estimates:

$$
\mathcal{R}_{\mathcal{L}\left(L_{q}\left(\mathbb{R}_{+}^{N}\right)^{N}, L_{q}\left(\mathbb{R}_{+}^{N}\right)^{2 N+N^{2}+N^{3}}\right)}\left(\left\{\left(\tau \partial_{\tau}\right)^{\ell}\left(G_{\lambda} \mathcal{V}_{+}(\lambda)\right) \mid \lambda \in \Gamma_{\epsilon, \lambda_{0}}\right\}\right) \leq C \quad(\ell=0,1)
$$

with some constant $C$ depending on $\epsilon, \lambda_{0}, \delta_{0}, \mu_{+}, \nu_{+}, \gamma_{0_{+}}, \gamma_{+1}, \gamma_{+2}, q$ and $N$.

Theorem 6.2 Let $1<q<\infty$ and $0<\epsilon<\pi / 2$. Then there exist operator families

$$
\begin{aligned}
& \mathcal{V}_{-}(\lambda) \in \operatorname{Hol}\left(\Sigma_{\epsilon}, \mathcal{L}\left(L_{q}\left(\mathbb{R}_{-}^{N}\right)^{N}, W_{q}^{2}\left(\mathbb{R}_{-}^{N}\right)^{N}\right)\right), \\
& \mathcal{O}_{-}(\lambda) \in \operatorname{Hol}\left(\Sigma_{\epsilon}, \mathcal{L}\left(L_{q}\left(\mathbb{R}_{-}^{N}\right)^{N}, \hat{W}_{q}^{1}\left(\mathbb{R}_{-}^{N}\right)\right)\right)
\end{aligned}
$$

such that for any $\lambda \in \Sigma_{\epsilon}$ and $\mathbf{f}_{-} \in L_{q}\left(\mathbb{R}_{-}^{N}\right)^{N}, \mathbf{w}_{-}=\mathcal{V}_{-}(\lambda) \mathbf{f}_{-}$and $\theta_{-}=\mathcal{O}_{-}(\lambda) \mathbf{f}_{-}$are unique solutions to problem (6.2), and $\mathcal{V}_{-}(\lambda)$ and $\mathcal{O}_{-}(\lambda)$ satisfy the following estimates:

$$
\begin{aligned}
& \mathcal{R}_{\mathcal{L}\left(L_{q}\left(\mathbb{R}_{-}^{N}\right)^{N}, L_{q}\left(\mathbb{R}_{-}^{N}\right)^{\left.2 N+N^{2}+N^{3}\right)}\right.}\left(\left\{\left(\tau \partial_{\tau}\right)^{\ell} G_{\lambda} \mathcal{V}_{-}(\lambda) \mid \lambda \in \Sigma_{\epsilon}\right\}\right) \leq C \quad(\ell=0,1), \\
& \mathcal{R}_{\mathcal{L}\left(L_{q}\left(\mathbb{R}_{-}^{N}\right)^{N}, L_{q}\left(\mathbb{R}_{-}^{N}\right)^{N}\right)}\left(\left\{\left(\tau \partial_{\tau}\right)^{\ell} \nabla \mathcal{O}_{-}(\lambda) \mid \lambda \in \Sigma_{\epsilon}\right\}\right) \leq C \quad(\ell=0,1)
\end{aligned}
$$

with some constant $C$ depending on $\epsilon, \mu_{-}, \gamma_{0_{-}}, q$ and $N$.

The composite operator of two $\mathcal{R}$-bounded operators is $\mathcal{R}$-bounded and the sum of two $\mathcal{R}$-bounded operators is also $\mathcal{R}$-bounded. Extending the operator $\mathcal{V}_{+}(\lambda)$ to $\mathbb{R}_{-}^{N}$ and the operators $\mathcal{V}_{-}(\lambda)$ and $\mathcal{O}_{-}(\lambda)$ to $\mathbb{R}_{+}^{N}$ by the PL Lions method, respectively, we see that the resulting operators also $\mathcal{R}$-bounded, so that combining Theorem 6.1 and Theorem 6.2 with (2.38), we have Theorem 1.2. This completes the proof of Theorem 1.2. 


\section{Author details}

1 Division of Mathematics, Faculty of Pure and Applied Sciences, University of Tsukuba, 1-1-1 Tennoudai, Tsukuba-shi, Ibaraki, 305-8571, Japan. ²Department of Mathematics, Research Institute of Science and Engineering, Waseda University, Ohkubo 3-4-1, Shinjuku-ku, Tokyo, 169-8555, Japan. ${ }^{3}$ CNRS-ENS Lyon, UMPA UMR 5669, Lyon cedex 7, 69364, France.

\section{Acknowledgements}

Second author was partially supported by JST CREST, JSPS Grant-in-aid for Scientific Research (S)\#24224004, and the JSPS Japanese-German Graduate Externship.

\section{Received: 3 March 2014 Accepted: 27 May 2014 Published online: 24 September 2014}

\section{References}

1. Denisova, IV: Evolution of compressible and incompressible fluids separated by a closed interface. Interfaces Free Bound. 2(3), 283-312 (2000)

2. Denisova, IV: A priori estimates of the solution of a linear time-dependent problem connected with the motion of drop in a fluid medium. Tr. Mat. Inst. Steklova 188, 3-21 (1990); English transl. in Proc. Steklov Inst. Math., 1-24 (1991)

3. Denisova, IV: Problem of the motion of two viscous incompressible fluids separated by a closed free interface. Acta Appl. Math. 37, 31-40 (1994)

4. Denisova, IV, Solonnikov, VA: Solvability of the linearized problem on the motion of a drop in a liquid flow. Zap. Nauč. Semin. LOMI 171, 53-65 (1989); English transl. in J. Sov. Math. 56, 2309-2316 (1991)

5. Denisova, IV, Solonnikov, VA: Classical solvability of the problem of the motion of two viscous incompressible fluids Algebra Anal. 7, 101-142 (1995); English transl. in St. Petersburg Math. J. 7, 755-786 (1996)

6. Prüß, J, Shibata, Y, Shimizu, S, Simonett, G: On well-posedness of incompressible two-phase flows with phase transitions: the case of equal densities. Evol. Equ. Control Theory 1, 171-194 (2012)

7. Prüß, J, Shimizu, S: On well-posedness of incompressible two-phase flows with phase transitions: the case of non-equal densities. J. Evol. Equ. 12,917-941 (2012)

8. Prüß, J, Simonett, G: On the two-phase Navier-Stokes equations with surface tension. Interfaces Free Bound. 12 311-345 (2010)

9. Prüß, J, Simonett, G: Analytic solutions for the two-phase Navier-Stokes equation with surface tension and gravity. Prog. Nonlinear Differ. Equ. Appl. 80, 507-540 (2011)

10. Shibata, Y, Shimizu, S: On resolvent estimate of the interface problem for the Stokes system in a bounded domain J. Differ. Equ. 191, 469-519 (2003)

11. Shibata, $Y$, Shimizu, S: Maximal $L_{p}-L_{q}$ regularity for the two-phase Stokes equations; model problems. J. Differ. Equ. 251, 373-419 (2011)

12. Denisova, IV: Problem of the motion of two compressible fluids separated by a closed free interface. Zap. Nauč Semin. POMI 243, 61-86 (1997); English transl. in J. Math. Sci. 99, 837-853 (2000)

13. Tani, A: Two phase free boundary problem for compressible viscous fluid motion. J. Math. Kyoto Univ. 24, 243-267 (1984)

14. Weis, L: Operator-valued Fourier multiplier theorems and maximal $L_{p}$-regularity. Math. Ann. 319, 735-758 (2001)

15. Kubo, T, Shibata, Y: On the evolution of compressible and incompressible viscous fluids with a sharp interface. Preprint (2013)

16. Shibata, Y, Tanaka, $K$ : On a resolvent problem for the linearized system from the dynamical system describing the compressible viscous fluid motion. Math. Methods Appl. Sci. 27, 1579-1606 (2004)

17. Enomoto, Y, Shibata, Y: On the $\mathcal{R}$-sectoriality and its application to some mathematical study of the viscous compressible fluids. Funkc. Ekvacioj 56(3), 441-505 (2013)

18. Shibata, Y: On the $\mathcal{R}$-boundedness of solution operators for the Stokes equations with free boundary condition. Differ. Integral Equ. 27, 313-368 (2014)

19. Shibata, $Y$, Shimizu, S: On the $L_{p}-L_{q}$ maximal regularity of the Stokes problem with first order boundary condition; model problem. J. Math. Soc. Jpn. 64(2), 561-626 (2012)

20. Shibata, Y, Shimizu, S: A decay property of the Fourier transform and its application to the Stokes problem. J. Math. Fluid Mech. 3(3), 213-230 (2001)

21. Götz, D, Shibata, Y: On the $\mathcal{R}$-boundedness of the solution operators in the study of the compressible viscous fluid flow with free boundary condition. Asymptot. Anal. (to appear). doi:10.3233/ASY-141238

doi:10.1186/s13661-014-0141-3

Cite this article as: Kubo et al.: On the $\mathcal{R}$-boundedness for the two phase problem: compressible-incompressible

model problem. Boundary Value Problems 2014 2014:141. 